\title{
Targeting BET Bromodomains in Recalcitrant Cancers
}

\author{
Sheena Hazel Clift \\ McLean, Virginia
}

B.S., Christopher Newport University, 2009

M.S., University of Virginia, 2012

A Dissertation presented to the Graduate Faculty of the University of Virginia in

Candidacy for the Degree of Doctor of Philosophy

Department of Biochemistry and Molecular Genetics

University of Virginia

April 2016 


\section{Abstract}

Pancreatic and lung cancers have some of the worst five-year survival rates of all malignancies. These aggressive cancers are often diagnosed as metastatic disease and are refractory to therapeutic interventions. Driving mutations in these diseases are often therapeutic targets, but cancer cells evade death by circumventing the pathways targeted by drugs. In order to do this, tumor cells will often undergo transcriptional reprogramming events, such as epithelial-tomesenchymal transition (EMT). Transcriptional reprogramming relies heavily on epigenetic reprogramming to define active enhancers and promoters for transcriptional activation. Bromodomain extraterminal domain (BET) proteins associate with enhancers and promoters to coordinate transcriptional activation of genes. We hypothesized that inhibition of BET proteins will disrupt the transcriptional reprogramming associated with EMT and drug resistance in cancer. In combination with targeted therapy against the mitogen-activated protein kinase (MAPK) pathway, BET inhibition results in increased apoptosis and decreased expression of genes associated with EMT and stemness in pancreatic cancer cell cultures. Based on histological analysis of an in vivo xenograft model of pancreatic cancer, we show that combined MAPK pathway and BET inhibition is more effective in inducing tumor regression than MAPK pathway inhibition alone. In an EMT model of lung cancer, BET inhibition markedly decreased the ability of cells to migrate and invade. In this dissertation, I present evidence of the therapeutic value of using BET inhibitors to treat pancreatic and lung adenocarcinomas. 


\section{Acknowledgements}

It is with tremendous support from a number of individuals that I am able to present the work in this dissertation. First, I would like to thank my doctoral advisor, Dr. Marty Mayo, for taking on a really inexperienced scientist and allowing me to explore numerous projects in the lab. I am really appreciative of

the years of advice and support that he's provided me. Additionally, I extend my gratitude to members of my thesis committee, Drs. Stefan Bekiranov, Todd Bauer, Tom Parsons, and Joel Hockensmith, for insightful feedback throughout my time at the University of Virginia (UVA). I would also like to thank the Cancer Training Grant and the UVA Cancer Center for supporting the work presented in this dissertation.

I have relied heavily on my labmates for technical advice, help with experimental design, favors, and friendship. I would like to particularly thank former lab members David Allison and Jake Wamsley for taking me under wing during my first few years at UVA. Not only did they show me protocols and techniques necessary to conduct my research, they also taught me to think scientifically. Lisa Gray has been an advocate for my success over the past six years, and works thoughtfully to keep the lab running smoothly. Additional lab members that have supported me include Julia Krupa, Szymon Szymura, Lisa Shock, and Brian McKenna. I would also like to thank the department of Biochemistry and Molecular Genetics for providing an energetic and collaborative environment in which to conduct science. 
Most importantly, I would like to acknowledge my family for a lifetime of love and support. I am grateful to my parents, who have worked tirelessly to provide me with limitless opportunity to explore the world and find my own success. Growing up with my older brother, Nick, equipped me a sense of adventure and the ability to take risks - characteristics necessary for a career in science. I am thankful to my in-laws, who have been incredibly enthusiastic and supportive of my graduate education. I must thank my four-legged bundles of unconditional love, Zoey and Bobby, who remind me that bad days (and even good days) can be made better with a long walk and a cuddle. Of paramount significance, I wholeheartedly thank my husband Jason for all that he does. Daily, and without expectation, he strives to make me feel loved, supported and happy. 
Table of Contents

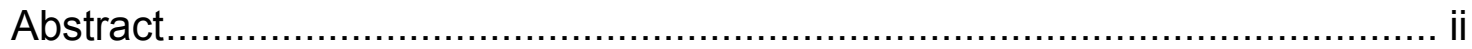

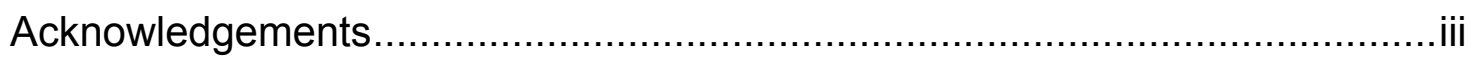

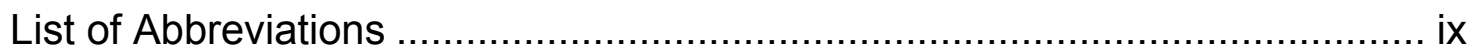

List of Tables \& Figures ........................................................................

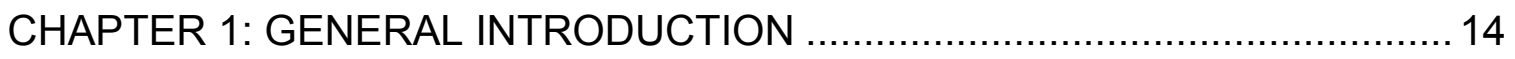

Targeting BET Bromodomains in Recalcitrant Cancers .............................. 15

EMT Drives Cancer Progression by Enriching the CIC Population ................ 18

EMT and $\mathrm{ClC}$ correlate with poor prognosis and drug resistance in aggressive

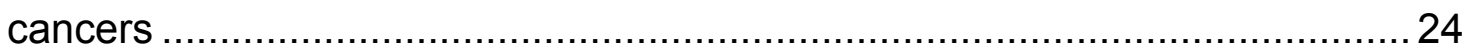

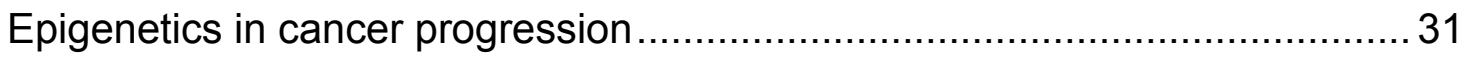

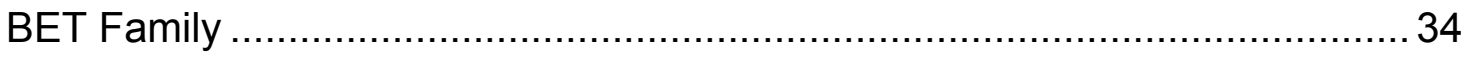

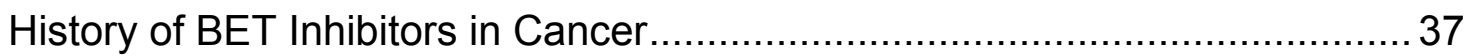

CHAPTER 2: MATERIALS \& METHODS ……........................................ 43

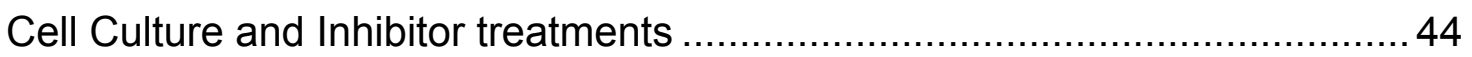

Three-Dimensional (3D) Multicellular Tumor Spheres.................................. 44

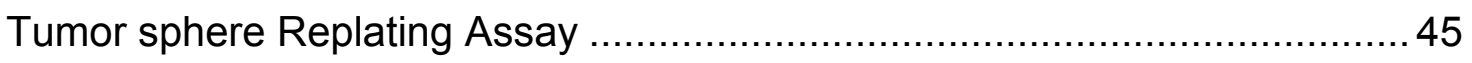

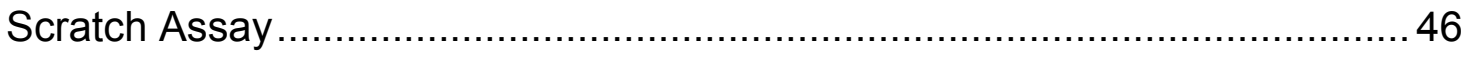

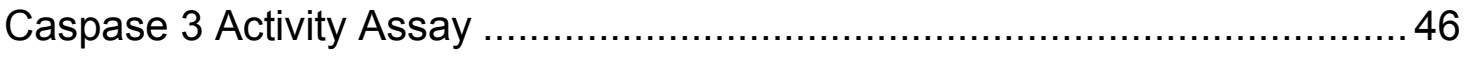

Orthotopic Xenograft Tumor Model and Histopathology............................... 47

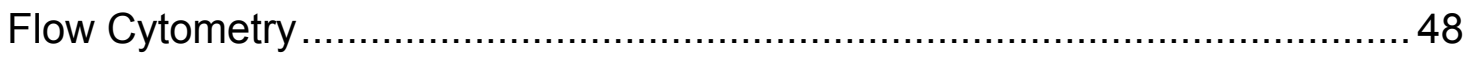

Quantitative Real-Time Polymerase Chain Reaction (QRT-PCR)..................49

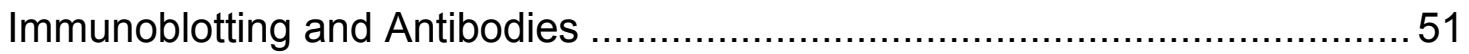




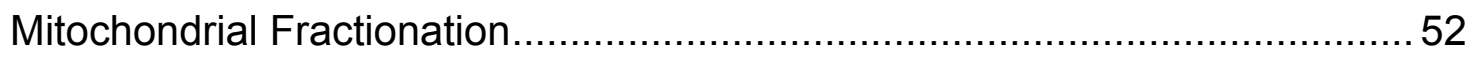

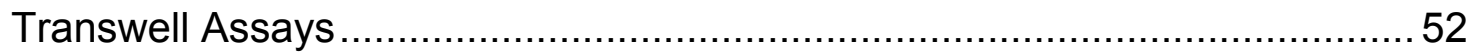

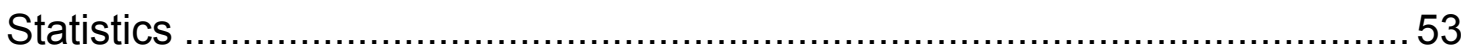

CHAPTER 3: TRAMETINIB AND I-BET151 AS A NOVEL COMBINATORIAL

THERAPY FOR PANCREATIC ADENOCARCINOMA .................................... 54

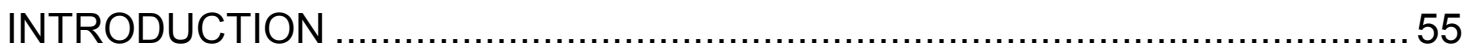

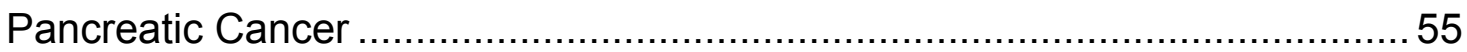

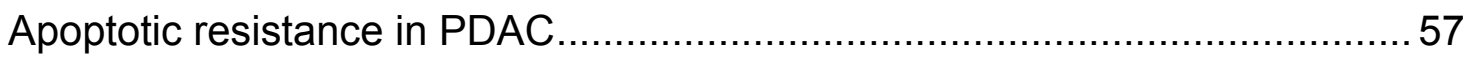

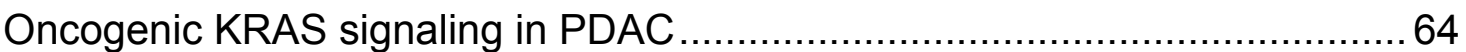

A Novel Combinatorial Therapy for PDAC: trametinib and I-BET151 .............67

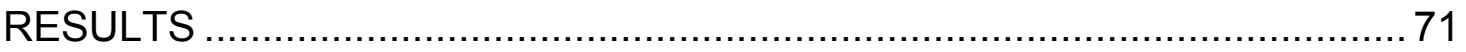

Trametinib treatment of PDAC tumor spheres selects for a refractory

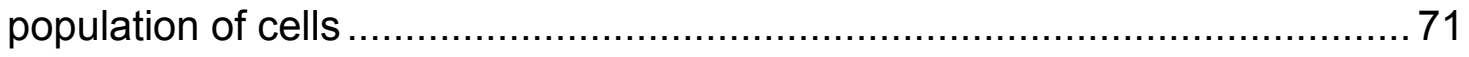

Combination of I-BET151 and trametinib synergize to reduce the population of refractory PDAC cells in tumor sphere culture .......................................... 78 Addition of I-BET151 increases trametinib-induced apoptosis in tumor spheres

Combinatorial treatment reduces markers of EMT and CIC in PDAC cells.....92 Treatment of orthotopic PDAC tumors with trametinib and I-BET151 induces

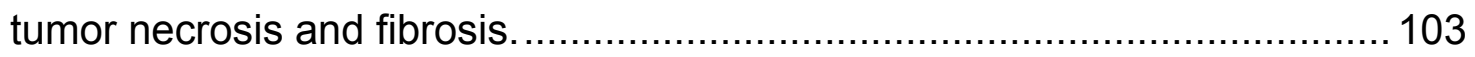

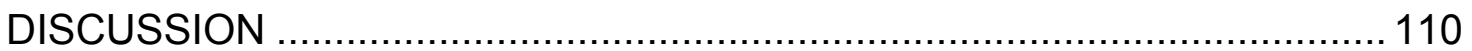

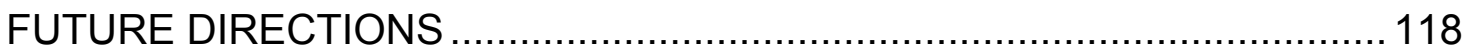


Combinatorial therapy increases overall survival in an in vivo xenograft model

Combinatorial therapy reduces outgrowth in an occult metastatic tumor model

CHAPTER 4: BET INHIBITION IMPEDES MIGRATION AND INVASION

PHENOTYPES IN NON-SMALL CELL LUNG CANCER .............................. 123

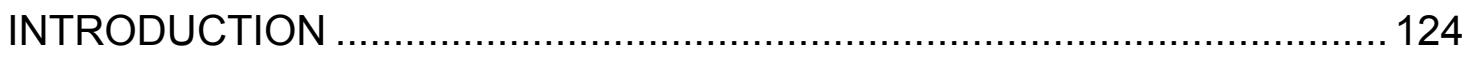

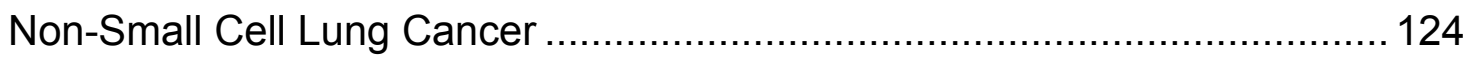

Risk Factors and Prognosis for Non-Small Cell Lung Cancer ...................... 125

Molecular Signatures and Targeted Therapy for NSCLC ........................... 126

A Rationale for Targeting EMT in NSCLC …....................................... 128

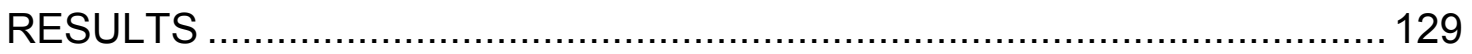

JQ1 inhibits expression of EMT master-switch transcription factors in an EMT

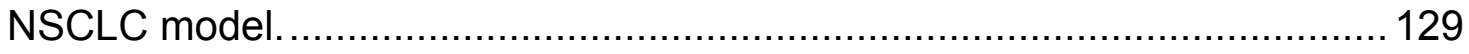

JQ1 inhibits the invasive and migratory capacity of cytokine treated A549

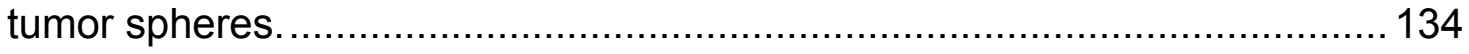

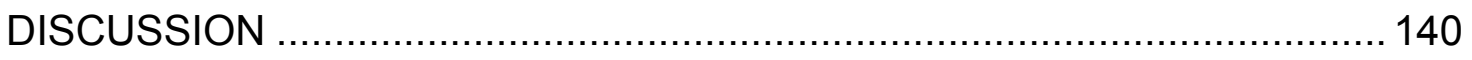

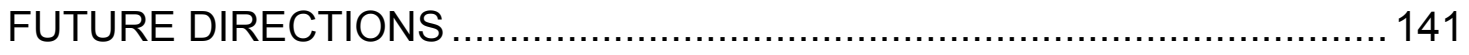

Evaluating the ability of BET inhibition to block metastasis in a NSCLC model

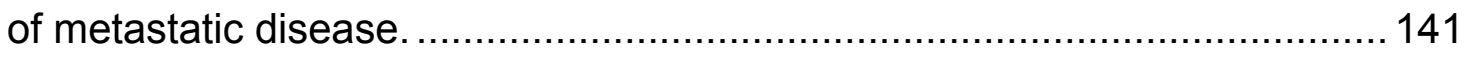

CHAPTER 5: GENERAL DISCUSSION \& FUTURE DIRECTIONS ................ 144

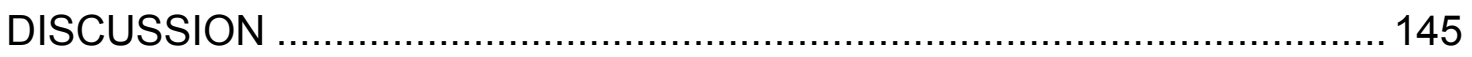

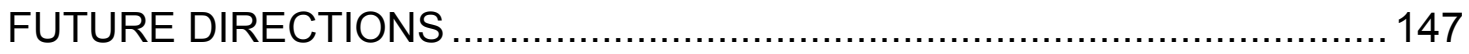


Confirm the effect of BET inhibition on the disruption of super-enhancers in

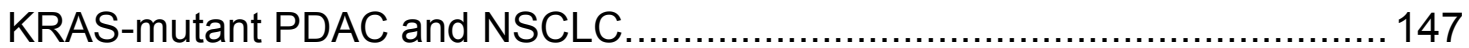

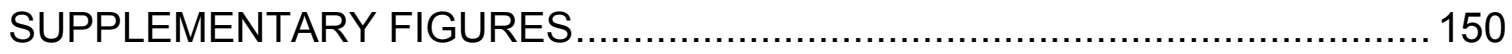

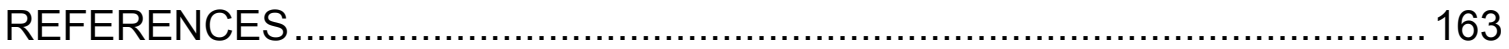




\section{List of Abbreviations}

$\begin{array}{ll}\text { ABC } & \text { ATP-binding cassette transporters } \\ \text { ABCB1 } & \text { ATP-binding cassette subfamily B member 1 } \\ \text { ABCC1 } & \text { ATP-binding cassette subfamily C member 1 } \\ \text { ABCG2 } & \text { ATP-binding cassette subfamily G member 2 } \\ \text { ADM } & \text { Acinar-ductal metaplasia } \\ \text { ALL } & \text { Acute lymphoblastic leukemia } \\ \text { AML } & \text { Acute myeloid leukemia } \\ \text { APAF1 } & \text { Apoptotic peptidase activating factor 1 } \\ \text { BAD } & \text { BCL-2-associated death promoter } \\ \text { BAK } & \text { BCL-2 homologous antagonistic killer } \\ \text { BAX } & \text { BCL-2-associated X protein } \\ \text { BCL-2 } & \text { B-cell CLL/lymphoma 2 } \\ \text { BCL-XL } & \text { B-cell lymphoma-extra large; BCL-2 family member } \\ \text { BET } & \text { Bromodomain extraterminal domain family of protein } \\ \text { BID } & \text { BH3 interacting-domain death agonist } \\ \text { BIM } & \text { BCL-2-interacting mediator } \\ \text { BIMEL } & \text { BCL-2-interacting mediator, extra long isoform } \\ \text { BIML } & \text { BCL-2-interacting mediator, long isoform } \\ \text { BIMS } & \text { BCL-2-interacting mediator, short isoform } \\ \text { BL } & \text { Burkitt's lymphoma } \\ \text { BMP2 } & \text { Bone morphogenic protein 2 } \\ \text { BMP7 } & \text { Bone morphogenic protein 7 } \\ \text { BRAF } & \text { B-Raf proto-oncogene, serine/threonine kinase } \\ \text { BRD } & \text { Bromodomain } \\ \text { BRD2 } & \text { Bromodomain containing protein 2 } \\ \text { BRD3 } & \text { Bromodomain containing protein 3 } \\ \text { BRD4 } & \text { Bromodomain containing protein 4 } \\ \text { BRDT } & \text { Bromodomain containing protein, testis-specific } \\ \text { CBP } & \text { CREB-binding protein } \\ \text { CD133 } & \text { CD133 antigene aka prominin-1: Cell-surface glycoprotein and } \\ & \text { stemness marker } \\ \text { CD24 } & \text { CD24 antigen: Cell-surface protein that modulates growth and } \\ & \text { differentiation signals } \\ \text { CD44 } & \text { CD44 antigen: Cell-surface glycoprotein involved in cell-cell } \\ & \text { interactions, cell adhesion and migration. } \\ \text { CDH1 } & \text { Cadherin-1 aka E-cadherin (epithelial) } \\ \text { CDH2 } & \text { Cadherin-2 aka N-cadherin (neuronal) } \\ \text { CDKN2A } & \text { Cyclin-dependent kinase inhibitor 2A } \\ \text { CHOP-C } & \text { C/EBP homologous protein C } \\ \text { CIC } & \text { Cancer-initiating cells } \\ \text { CXCL8 } & \text { Chemokine C-X-C motif ligand 8 } \\ \text { DAPI } & \text { 4',6-diamidino-2-phenylindole; a fluorescent stain that binds DNA } \\ \text { DR3 } & \text { Death receptor 3 } \\ \text { DR4 } & \text { Death receptor 4 } \\ & \\ & \end{array}$




$\begin{array}{ll}\text { DRP1 } & \text { Dynamin-related protein 1 } \\ \text { E2F1 } & \text { E2F transcription factor 1 } \\ \text { EBV } & \text { Epstein-Barr virus } \\ \text { ECM } & \text { Extracellular Matrix } \\ \text { EGF } & \text { Epidermal growth factor } \\ \text { EGFR } & \text { Epidermal growth factor receptor } \\ \text { EMT } & \text { Epithelial-to-mesenchymal transition } \\ \text { EpCAM } & \text { Epithelilal cell adhesion molecule } \\ \text { ER } & \text { Endoplasmic reticulum } \\ \text { ERBB3 } & \text { Erb-B2 receptor tyrosine kinase 3 } \\ \text { ERK } & \text { Extracellular signal-regulated kinase } \\ \text { ESA } & \text { Epithelial-specific antigen } \\ \text { ET } & \text { Extraterminal domain } \\ \text { EZH2 } & \text { Enhancer of zeste homolog 2 } \\ \text { FACS } & \text { Fluoresence-activated cell sorting } \\ \text { FAP1 } & \text { Familial adenomatous polyposis-1 } \\ \text { FasL } & \text { FAS ligand } \\ \text { FasR } & \text { FAS receptor } \\ \text { FGF } & \text { Fibroblast growth factor } \\ \text { FOSL1 } & \text { FOS like antigen 1 } \\ \text { FOXO3a } & \text { Forkhead box O3 } \\ \text { GAPDH } & \text { Glyceraldehyde 3-phosphate dehydrogenase } \\ \text { GATA1 } & \text { GATA binding protein 1 } \\ \text { GLIS3 } & \text { Gli-similar 3 } \\ \text { GSK } & \text { GlaxoSmithKline } \\ \text { GTPase } & \text { Guanosine triphosphate hydrolase } \\ \text { HAT } & \text { Histone acetyltransferase } \\ \text { HDAC } & \text { Histone deacetylase } \\ \text { HER2 } & \text { Human epidermal growth factor receptor 2 } \\ \text { HGF } & \text { Hepatocyte growth factor } \\ \text { Hh } & \text { Hedgehog } \\ \text { HIF } & \text { Hypoxia-inducible factor } \\ \text { HMT } & \text { Histone methyltransferase } \\ \text { HPV } & \text { Human papillomavirus } \\ \text { IKBa } & \text { Inhibitor of kappa B alpha } \\ \text { JNK } & \text { Jun N-terminal kinase } \\ \text { KLF4 } & \text { Kruppel-like factor 4 } \\ \text { KRAS } & \text { Kirsten rat sarcoma viral oncogene homolog } \\ \text { LC3A/B } & \text { Microtubule-associated protein light chain 3 A and B } \\ \text { MAPK } & \text { Mitogen-activated protein kinase } \\ \text { MEK } & \text { Mitogen-activated protein kinase kinase (MAPKK) } \\ \text { MET } & \text { Mesenchymal-to-epithelial transition } \\ \text { MET } & \text { MNNG-HOS transforming gene } \\ \text { MM } & \text { Multiple myeloma } \\ \text { MOM } & \text { Mitochondrial outer membrane } \\ \text { MRI } & \text { Magnetic Resonance Imaging } \\ & \end{array}$




$\begin{array}{ll}\text { MSC } & \text { Mesenchymal stem cell } \\ \text { NF-kB } & \text { Nuclear factor kappa B } \\ \text { NMC } & \text { NUT (Nuclear protein in testis gene) midline carcinoma } \\ \text { NOXA } & \text { BH3-only protein in the BCL-2 family of proteins } \\ \text { NSCLC } & \text { Non-small cell lung cancer } \\ \text { OCT4 } & \text { Octamer-Binding Protein 4 aka POU Class 5 Homeobox 1 } \\ & \text { (POU5F1) } \\ \text { OS } & \text { Overall survival } \\ \text { P-TEFb } & \text { Positive transcription elongation factor b } \\ \text { Panln } & \text { Pancreatic intraepithelial neoplasia } \\ \text { PARP } & \text { Poly ADP ribose polymerase } \\ \text { PCAF } & \text { P300/CBP-associated factor } \\ \text { PDAC } & \text { Pancreatic ductal adenocarcinoma } \\ \text { PFS } & \text { Progression-free survival } \\ \text { PI3K } & \text { Phosphoinositide 3-kinase } \\ \text { Pol II } & \text { RNA polymerase II } \\ \text { PUMA } & \text { P53 upregulated mediator of apoptosis } \\ \text { qRT-PCR } & \text { Quantitative reverse transcription polymerase chain reaction } \\ \text { ROS } & \text { Reactive oxygen species } \\ \text { RTK } & \text { Receptor tyrosine kinase } \\ \text { RUNX3 } & \text { Runt-related transcription factor 3 } \\ \text { SCC } & \text { Squamous cell carcinoma, a subtype of lung cancer } \\ \text { SCLC } & \text { Small-cell lung cancer, a subtype of lung cancer } \\ \text { SHH } & \text { Sonic Hedgehog } \\ \text { shRNA } & \text { Small hairpin RNA } \\ \text { siRNA } & \text { Small interfering RNA } \\ \text { SMAD4 } & \text { SMAD family member 4 } \\ \text { SNAI1 } & \text { Snail family zinc finger 1; encodes Snail protein } \\ \text { SNAI2 } & \text { Snail family zinc finger 2; encodes Slug protein } \\ \text { SOX2 } & \text { SRY (sex determining region Y)-box 2 } \\ \text { SRp55 } & \text { Splicing factor arginine/serine-rich 6 aka SRSF6 } \\ \text { TAF } & \text { TBP associated factor } \\ \text { TBP } & \text { TATA box binding protein } \\ \text { TGF } & \text { Tumor growth factor beta } \\ \text { TKI } & \text { Tyrosine kinase inhibitor } \\ \text { TNF } & \text { Tumor necrosis factor } \\ \text { TNFR1 } & \text { Tumor necrosis factor receptor 1 } \\ \text { TRAIL } & \text { TNF-related apoptosis inducing ligand } \\ \text { TSS } & \text { Transcription start site } \\ \text { TWEAK } & \text { TNF-related weak inducer of apoptosis } \\ \text { TWIST1 } & \text { Twist family BHLH transcription factor 1 } \\ \text { VEGF } & \text { Vascular endothelial growth factor } \\ \text { Wnt } & \text { Wingless tail } \\ \text { ZEB2 } & \text { Zinc finger E-box binding homeobox2 } \\ & \\ & \end{array}$




\section{List of Tables \& Figures}

Table 1. Overall summary of US disease occurrence, mortality and frequency of

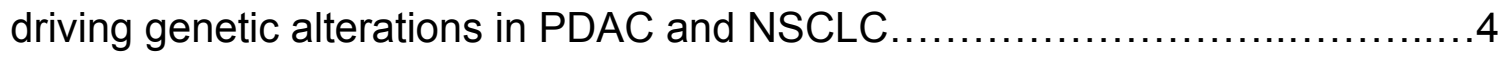

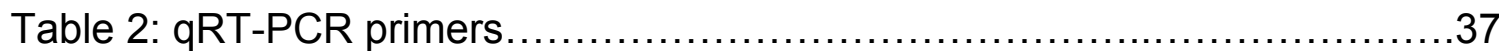

Figure 1. Characteristics of the Epithelial-to-Mesenchymal Transition............14

Figure 2. Genetic and epigenetic reprogramming contributes to drug resistance and disease recurrence in recalcitrant cancers..................................16

Figure 3. Schematic of BET protein function in transcriptional activation.........28

Figure 4. BIM-Induced Apoptosis ...........................................49

Figure 5. Hypothesis: Trametinib and I-BET151 as a Novel Combinatiorial

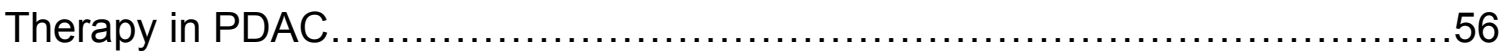

Figure 6. PDAC tumor spheres exhibit resistance to trametinib.................61

Figure 7. I-BET151 treatment increases sensitivity of PDAC cells to trametinib.

Figure 8. Combinatorial treatment with trametinib and I-BET151 induces

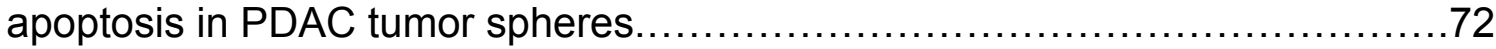

Figure 9. Individual and combinatorial treatments of trametinib and/or I-BET151

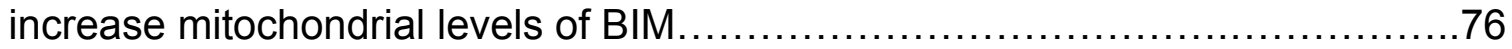

Figure 10. Combinatorial treatment reduces mesenchymal markers and migration phenotype in PDAC cells.

Figure 11. Combinatorial treatment reduces pluripotency markers in tumor

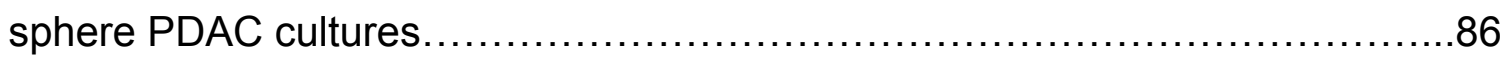


Figure 12: I-BET151 increases tumor necrosis in trametinib-treated orthotopic PDAC model.

Figure 13. JQ1 inhibits expression of EMT master-switch transcription factors in TNF/TGF $\beta$-stimulated tumor sphere NSCLC cultures 118

Figure 14. JQ1 impedes migration and invasion in tumor sphere NSCLC cultures 123

Figure S1. Knockdown of BIM using siRNA partially rescues the apoptotic phenotype. 138

Figure S2. The super repressor of NF-kB fails to induce expression of BIM in 366 and 608 PDAC cells. 140

Figure S3. Trametinib and I-BET151 increase mitochondrial network connectivity in 2D PDAC cultures. 142

Figure S4. I-BET151 fails to induce apoptosis in DRP1 knockdown PDAC cells.

Figure S5. I-BET151 induces autophagy marker LC3A/B in 3D PDAC cultures.

Figure S6. I-BET151 alters histone acetylation profiles in 366 cells 
CHAPTER 1: GENERAL INTRODUCTION 


\section{Targeting BET Bromodomains in Recalcitrant Cancers}

In late January of 2013, the Recalcitrant Cancer Research Act was signed into law. The bill instructs the National Cancer Institute to accelerate research on cancers that take the lives of more than 30,000 Americans annually and have a five-year survival rate below 20\% (Eshoo, 2012). Both lung and pancreatic cancers meet these criteria as recalcitrant malignancies, and have been the focus of my studies while at the University of Virginia. Specifically, I have been studying adenocarcinoma subtypes of lung and pancreatic cancers: non-small cell lung adenocarcinoma (NSCLC) and pancreatic ductal adenocarcinoma (PDAC).

Both NSCLC and PDAC are heterogeneous diseases driven by genetic mutations. Many of these mutations result in the activation of gene products that promote processes essential to tumor development and progression, such as proliferation, angiogenesis, metastasis, and evasion of apoptosis. Tumors harboring these driving mutations are thought to be "addicted" to the constitutive activity of the gene products, and require their hyperactivity for maintenance, growth and survival. In addition to poor outcome, PDAC and NSCLC are similar in that they are both commonly initiated and potentiated by activating mutations in the KRAS oncogene (Table 1). These transforming mutations in KRAS constitutively drive proliferative expansion and survival signals through the MEK/ERK and PI3K pathways (Fang, 2016; Pylayeva-Gupta et al., 2011). Persistent cell cycle progression can result in replicative stress-induced DNA damage and genomic instability that gives rise to an accumulation of mutations to 
potentiate tumorigenesis (Dobrila-Dintinjana et al., 2012). Furthermore, mutant KRAS can augment cell metabolism, angiogenesis, and immune evasion (Blum and Kloog, 2014; Kranenburg, 2005).

Tumor heterogeneity, metastasis, drug resistance and recurrence of disease contribute to the low survivability of recalcitrant cancers (Quinn et al., 2015). All of these properties rely on the ability of cells within the tumor to adapt to and interact with components of the tumor microenvironment. Metastasis, however, is responsible for causing ninety percent of cancer-related deaths (Kalluri and Weinberg, 2009; Kang and Massagué, 2004; Valastyan and Weinberg, 2011). The five-year survival rate for localized lung and pancreatic cancers are $65 \%$ and $22 \%$ respectively. These numbers precipitously drop below $15 \%$ for lung and $2 \%$ for pancreatic cancers involving distal metastasis (Ferlay et al., 2015; Jemal et al., 2011; Ryerson et al., 2016). The mechanisms underlying this process are of interest to cancer biologists, as are finding ways to impede metastatic progression.

In order to metastasize, tumor cells must acquire the ability to detach from the primary tumor, invade the basement membrane, travel through the circulatory system, exit the vasculature, and colonize a distal site (Frisch et al., 2013; Kang and Massagué, 2004). How do adenocarcinomas made up of epithelial-like cancer cells manage to accomplish this complex phenomenon? One wellsupported theory proposes that engagement of the epithelial-to-mesenchymal transition (EMT) program enables primary tumor cells to metastasize (Smith and Bhowmick, 2016). It has also been postulated, with evidentiary support, that 
EMT gives rise to a stem-like population that has the ability to differentiate during metastatic colonization and disease recurrence (D'Angelo and Wicha, 2010). Hereafter, these cancer stem-like cells will be referred to as cancer-initiating cells (CICs). The following sections will further discuss how EMT and $\mathrm{CICs}$ contribute to the mortality of recalcitrant cancers.

\begin{tabular}{|c|c|c|}
\hline & PDAC & NSCLC \\
\hline 5-year survival rate & $\sim 6 \%$ & $\sim 17 \%$ \\
\hline Estimated diagnoses (2016) & 49,000 & 225,000 \\
\hline Estimated deaths (2016) & 41,000 & 160,000 \\
\hline Projected diagnoses (2030) & 88,000 & 225,000 \\
\hline \multicolumn{2}{|c|}{ Genes w/ mutations, CNVs, or expression changes (\% frequency) } \\
\hline KRAS & $>90 \%$ (mutation) & $25 \%$ (mutation) \\
\hline P53 & $70 \%$ (loss) & $70 \%$ (loss) \\
\hline SMAD4 & $50 \%$ (loss) & $50 \%$ (loss, underexpression) \\
\hline CDKN2A/B & $50 \%$ (loss) & 23\% (loss) \\
\hline HER2/EGFR & $20 \%$ (mutation, overexpression) & $25 \%$ (mutation, amplification) \\
\hline
\end{tabular}

Table 1. Overall summary of US disease occurrence, mortality and frequency of driving genetic alterations in PDAC and NSCLC. (Ferlay et al., 2015; Rahib et al., 2014; American Cancer Society; 2016) Genetic alteration statistics based upon data generated by the TCGA Research Network: http://cancergenome.nih.gov/ 


\section{EMT Drives Cancer Progression by Enriching the CIC Population}

EMT is a naturally occurring process that is required for embryogenesis, development, wound healing, and tissue repair (Pasquier et al., 2015). It confers plasticity to polarized epithelial cells, allowing them to differentiate into fibroblastlike mesenchymal cells. This program is reversible via the mesenchymal-toepithelial transition (MET). Throughout embryogenesis, cycles of EMT and MET are required to form the mesoderm, neural crest, primary mesenchyme and secondary epithelium (Kalcheim, 2015). These EMT-MET cycles result in definition and organization of organs. EMT is also induced in response to tissue damage (Bellaye et al., 2014). Inflammation at the site of injury stimulates EMT to infiltrate the area with wound-healing fibroblasts that secrete extracellular matrix (ECM) components such as fibronectin and collagens in order to rebuild the tissue (Lamouille et al., 2014).

Pathophysiological activation of EMT has been described in numerous human diseases that are associated with inflammation. Under chronic inflammatory conditions, EMT and its associated production of fibroblasts occur in excess. Pathological EMT-associated fibrosis has been implicated in inflammatory diseases such as asthma, chronic obstructive pulmonary disorder, kidney disease, liver fibrosis, and other disorders (Bellaye et al., 2014; Rybinski et al., 2014; Tampe and Zeisberg, 2014). In cancer, however, it is not just the accumulation of fibroblasts that makes EMT problematic, rather the acquisition of an invasive and migratory phenotype in malignant cells. EMT is hypothesized to 
be the rate-limiting step during the metastatic process (Smith and Bhowmick, 2016).

A host of signals from the tumor microenvironment initiate EMT. These signals include cytokines and growth factors secreted by infiltrating immune cells and stromal cells, as well as the tumor cells themselves (Fuxe et al., 2010; Karin and Greten, 2005). One of the most potent inducers of EMT is transforming growth factor beta (TGF $\beta$ ), a pleiotropic growth factor that participates in various cellular processes (Vincent et al., 2009). Tumor necrosis factor (TNF) is another inflammatory cytokine that has been shown to promote EMT, especially in combination with TGF $\beta$ (Kumar et al., 2013).

Other growth factors and ligands that can regulate EMT include fibroblast growth factor (FGF), hepatocyte growth factor (HGF), epidermal growth factor (EGF), various interleukins, bone morphogenic protein 7 (BMP7), wingless tail (Wnt), hedgehog (Hh), Notch, and others (Huang et al., 2015; Smith and Bhowmick, 2016; Yoon et al., 2014). In addition to soluble factors, other aspects of the microenvironment can potentiate EMT. For example, hypoxia has been shown to induce expression of hypoxia-inducible factors (HIFs) that can promote EMT (Chang et al., 2011). The structure and density of the ECM and disruption of cell-cell or cell-ECM interactions can induce EMT (Shankar and Nabi, 2015). Induction of EMT leads to downstream activation of the EMT "masterswitch transcription factors" that propagate the transcriptional program. These master-switch transcription factors include zinc finger proteins Snail (SNA/1), Slug (SNAI2), and SIP1 (ZEB2) and basic helix-loop-helix protein Twist 
(TWIST1) (Bae et al., 2013; von Burstin et al., 2009; Mao et al., 2013; Vincent et al., 2009). TGF $\beta$ binds to cognate receptors to induce their dimerization and transphosphorylation. Stimulation of the receptor leads to phosphorylationmediated activation of Smad2/3, which then complexes with Smad4. The complex translocates to the nucleus to activate Snail and Slug, which in turn activates SIP1; these transcription factors then execute their regulatory functions (Lamouille et al., 2014; Zavadil and Böttinger, 2005). TNF-dependent signaling cascades stimulate NF-KB translocation, which is required to upregulate TWIST1 and SNAl1 expression. NF-KB signaling is associated with the production of CIC in prostate cancer (Odero-Marah et al., 2008). Our lab has extensively demonstrated the necessary role of NF-KB in the process of EMT and the production of CIC in NSCLC (Kumar et al., 2013; Wamsley et al., 2015).

Numerous studies have shown that activation of the KRAS/MEK/ERK pathway not only promotes proliferation, but also effects EMT through the master-switch transcription factors. In pancreatic cancer, mutant KRAS has been shown to stimulate EMT by activation of the $\mathrm{Wnt} / \beta$-catenin pathway which in turn increases expression of Snail and ZEB1 (Xu et al., 2015). In NSCLC, activation of MEK/ERK signaling induces expression of ZEB1 and MMP2, thus promoting invasive phenotypes (Bae et al., 2013). Additionally, bone morphogenetic protein 2 (BMP2) stimulates EMT in gastric cancer via the ERK signaling pathway (Liao et al., 2015). Of clinical relevance, KRAS/MEK/ERK driven EMT can give rise to $\mathrm{CIC}$ and drug resistance cell populations. Using a breast cancer model, Normanno and colleagues demonstrated that the MEK 
pathway is activated during acquired resistance to gefitinib, an EGFR tyrosine kinase inhibitor (Normanno et al., 2006).

In concert, the master-switch transcription factors repress genes encoding proteins that make up the epithelial phenotype, while activating genes governing the mesenchymal phenotype (Fig. 1). For instance, the zinc finger proteins bind to the E-boxes in the promoter of the $\mathrm{CDH} 1$ gene to repress expression of $\mathrm{E}$ cadherin (von Burstin et al., 2009). E-cadherin is a calcium dependent cell-cell adhesion protein integral to the maintenance of adherens junctions. Other epithelial proteins downregulated by these transcription factors include cytokeratins, claudins, and occuldins; these are involved in maintaining apicalbasal polarity, cell-cell junctions, and attachment of cells to the basement membrane (Kaufhold and Bonavida, 2014).

Mesenchymal proteins that are upregulated by the master-switch transcription factors facilitate cellular migration, remodeling of the ECM, and resistance to anoikis (Bae et al., 2013; Frisch et al., 2013; Hamilton et al., 2016; Kim et al., 2016). Examples of mesenchymal proteins include Vimentin, Fibronectin, N-cadherin, and matrix metalloproteinases (MMPs). NSCLC tumor cells that have transitioned through EMT display increased invasion phenotypes, and are able to establish tumors when injected into mice under limiting dilutions (Kumar et al., 2013). Additionally, cells that have undergone EMT alter their autocrine and paracrine signaling to maintain their mesenchymal phenotype, a property that is required for tumor cell circulation and metastatic tumor establishment (Wamsley et al., 2015). Together, these studies support the 
theory that mesenchymal cells are able to remodel ECM in order to intravasate into the circulatory system during metastatic progression.

EMT is a dedifferentiation program, and has been shown to confer multipotent properties to malignant cells (D'Angelo and Wicha, 2010; Pasquier et al., 2015). Numerous studies have shown correlation between the presence of EMT markers and CIC markers in cancer models (Blick et al., 2010; Mani et al., 2008). For example, $\mathrm{N}$-cadherin and Snail expression has been shown to positively correlate with expression of stem cell transcription factors SOX2, OCT4, and Nanog (Luo et al., 2013). Similarly, expression of EMT markers Snail and vimentin strongly correlates with CD44 expression, metastasis, and invasion (Ryu et al., 2012).

Much like normal "adult" stem cells, these stem-like cancer cells are resistant to apoptosis and have slow division kinetics. Conventional chemotherapies target rapidly dividing cells, missing or perhaps even selecting for these progenitor-like cells (Du et al., 2011). The drug-resistant populations have the ability to establish, maintain or regenerate a tumor; it is for this reason that they are also known as "cancer-initiating cells" (CICs). A CIC has the ability to adapt to secondary environments. Therefore, CICs that enter the circulatory system have the potential to disseminate to a distal site and initiate a secondary tumor lesion that maintains phenotypes found within the primary tumor (Kim et al., 2016; Saur et al., 2005). EMT induction of the CIC phenotype is believed to be key to metastatic disease progression. 
Herman et al., showed that as few as 500 patient-derived pancreatic cancer cells expressing $\mathrm{CIC}$ marker CD133 were sufficient to initiate orthotopic tumor formation when injected into athymic mice. This contrasts with no tumor formation when $1 \times 10^{6}$ CD133 negative cells were injected into mice. This study demonstrates the tumorigenicity, that is the ability to differentiate into a bulk tumor, of a small subset of cells within a heterogenic population (Hermann et al., 2007). Additionally, high concentrations of gemcitabine $(100 \mu \mathrm{g} / \mathrm{mL})$ were not effective in inducing apoptosis in this $\mathrm{CIC}$ population. Gemcitabine treatment of unsorted, orthotopically-implanted tumors shrank tumors, but also enriched for CD133+ cells by about 5 fold. These experiments show that conventional chemotherapies can select for these tumorigenic $\mathrm{CIC}$ cells, and that $\mathrm{CICs}$ demonstrate drug-resistant phenotypes.

Similarly, the Simeone lab showed that a subset of primary human PDAC cells could be FACS sorted to enrich for CIC cells expressing CD44, CD24, and epithelial-specific antigen (CD44+CD24+ESA+). As few as 100 of these CIC cells could faithfully form heterogeneous tumors if subcutaneously injected into NOD/SCID mice, which is in stark contrast to only $8 \%$ of mice forming tumors when injected with 10,000 unsorted cells. Although the triple positive CIC cells only made up $0.2-0.8 \%$ of the PDAC cell population in these patient derived tumors, they demonstrate enhanced tumorigenicity (Li et al., 2007). These CIC cells showed increased expression of Sonic Hedgehog (SHH), a pathway involved in developmental signaling. A different study demonstrated that inhibition of Hedgehog signaling, a pathway that maintains CIC phenotypes, 
could reverse chemotherapy resistance in CD44+ cells in gastric cancer (Yoon et al., 2014).

\section{EMT and CIC correlate with poor prognosis and drug resistance in}

\section{aggressive cancers}

While researchers have extensively described EMT and CIC in pre-clinical models, it has proven difficult to pinpoint in patient tumors due to the fact that EMT is a dynamic process within a heterogeneous tumor microenvironment. Mesenchymal tumor cells share characteristics with the surrounding stroma, making them difficult to distinguish from each other. Additionally, mesenchymal cells have the ability to differentiate into epithelial cells via MET, making their presence transient. Many studies, however, have shown that EMT gene expression and protein biomarkers strongly correlate with cancer progression, poor patient outcome, and therapy resistance (Arumugam et al., 2009; Smith and Bhowmick, 2016). A recent study showed that inhibition of EMT in a transgenic mouse model of PDAC sensitizes tumors to treatment with gemcitabine, thus providing an overall survival benefit (Zheng et al., 2015).

Patient outcome has been shown to correlate with EMT markers and an increased presence of mesenchymal circulating tumor cells in numerous cancer types (Hamilton et al., 2016). These cells demonstrate resistance to anoikis, or cell death caused by detachment from a basement membrane, a phenotype induced by EMT (Frisch et al., 2013). Notch signaling, an EMT regulatory pathway, is activated in gemcitabine-resistant pancreatic cancer cells (Wang et al., 2009). EMT masterswitch transcription factors Slug and Snail mediate 
chemoresistance and radioresistance in ovarian cancer through acquisition of stem-like phenotypes and inhibition of p53-mediated apoptosis (Kurrey et al., 2009). Ovarian cancer disease staging has been shown to correlate with upregulation of Twist and accumulation of nuclear $\beta$-catenin (Mao et al., 2013).

EMT has also been shown to upregulate the expression of ATP binding cassette $(A B C)$ family of proteins involved in conferring multidrug resistance (Długosz and Janecka, 2016). These proteins act as efflux pumps to export toxic agents from a cell. In NSCLC, expression of $A B C B 1$ is associated with acquired resistance to MNNG-HOS transforming gene (MET) inhibitors; this resistance can be overcome using siRNA against $A B C B 1$ or blocking $A B C B 1$ function with elacridar (Sugano et al., 2015). In chemoradiation-resistant pancreatic cancer cells, $A B C G 2$ expression was observed at higher levels compared to chemoradiation-sensitive cells. Additionally, these resistant cells expressed higher levels of anti-apoptotic proteins BCL-2 and Survivin, as well as stem cell markers OCT4, CD24, and CD133 (Du et al., 2011).

The presence of cells expressing $\mathrm{CIC}$ markers has been shown to correlate with decreased therapeutic response, increased metastasis and decreased survival. For example, enrichment for CD133+ cells in patient biopsies was predictive of poor radiochemotherapy response, shorter overall survival and increased distant cancer recurrence in colorectal cancer (Sprenger et al., 2013). Similarly, circulation of tumor cells expressing CD44 corresponded to increased lymph node metastasis, distant metastasis and accelerated disease recurrence in gastric cancer (Li et al., 2014). A phase II clinical trial testing the 
efficacy of chemotherapy in combination with vismodegib, an inhibitor of Hedgehog signaling, improved survival of patients whose clinical samples showed high expression of stemness marker CD44 (Yoon et al., 2014).

These and other studies stress the importance of monitoring these $\mathrm{CIC}$ populations before and during patient treatment. Additionally, therapeutic strategies to diminish $\mathrm{CIC}$ populations in addition to rapidly dividing cells are warranted. The ability of the CIC cells to establish heterogeneous tumors indicates a role for genetic and epigenetic reprogramming in metastasis. Blocking these dynamic reprogramming events by abrogating epigenetic factors is a potential strategy for limiting dedifferentiation, $\mathrm{CIC}$ formation, and metastatic progression through $\mathrm{CIC}$ differentiation into bulk tumor (Fig. 2). 


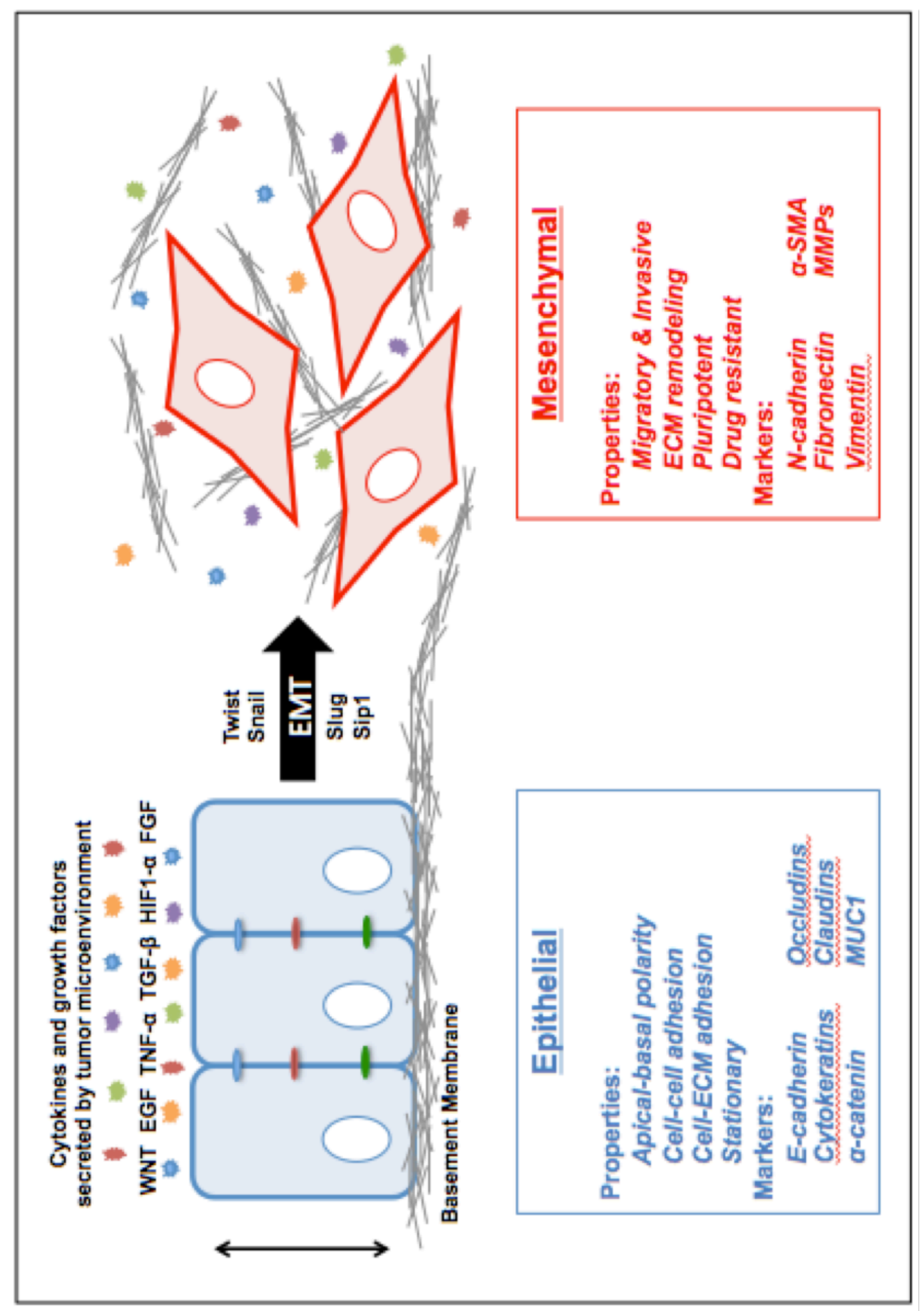


Figure 1. Characteristics of the Epithelial-to-Mesenchymal Transition. EMT is induced by growth factors and cytokines that are present in the tumor microenvironment. Upon binding to their respective receptors, these factors initiate signaling pathways that upregulate the expression of the master-switch transcription factors of EMT: Twist, Snail, Slug, and Sip1. These transcription factors, in turn, repress the expression of epithelial markers (blue box) and upregulate expression of mesenchymal markers (red box). Normal epithelial cells are characterized as being attached to neighboring cells as well as a basement membrane; they display polarity and are immobile. Mesenchymal cells are able detach from the basement membrane and migrate through the extracellular matrix. Malignant mesenchymal cells are multipotent and exhibit increased resistance to chemotherapy, radiotherapy, and several targeted therapies. Adapted from Krantz et al., 2010. 


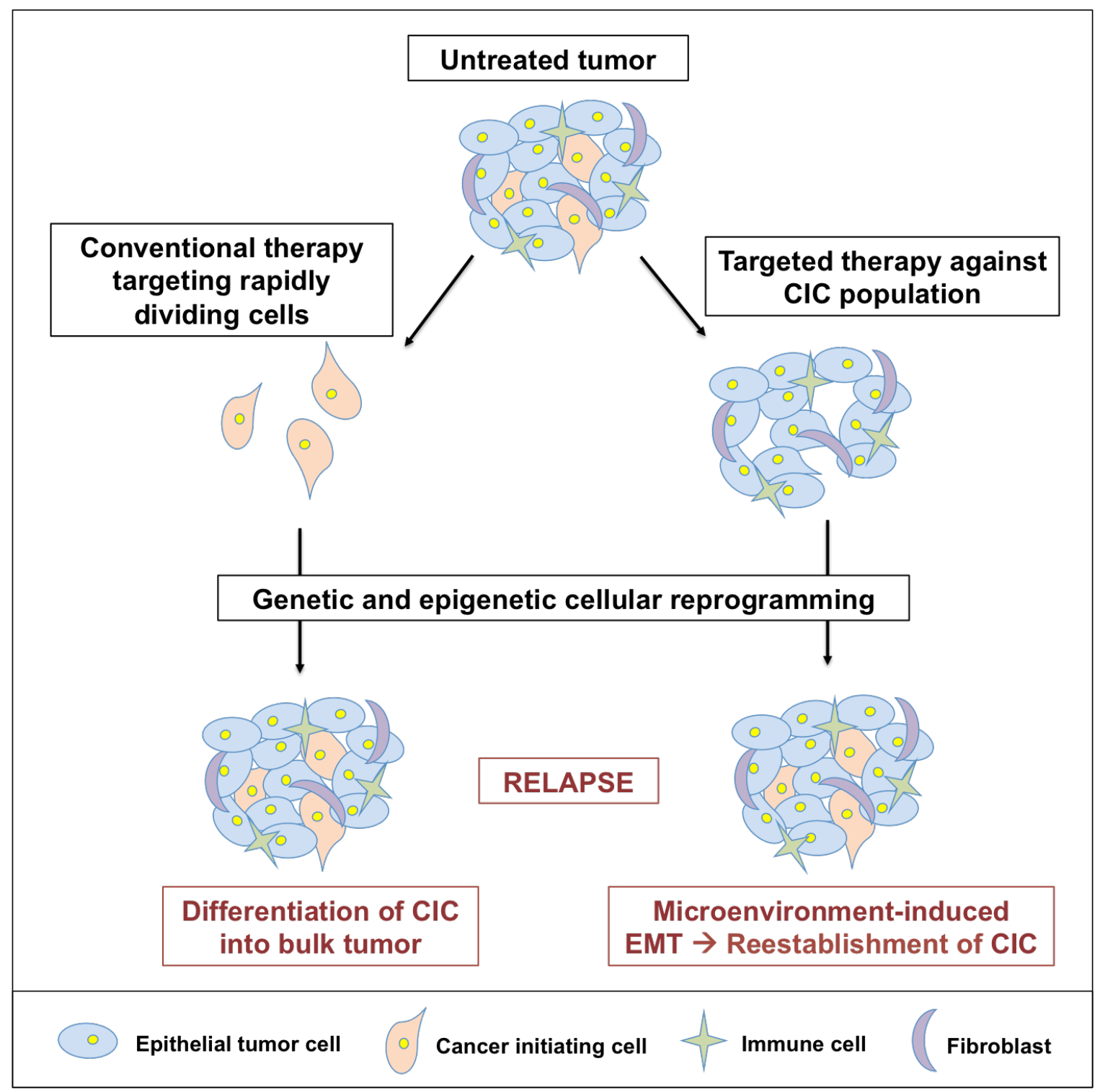


Figure 2. Genetic and epigenetic reprogramming contributes to drug resistance and disease recurrence in recalcitrant cancers. Conventional therapies target rapidly dividing cells, which in turn enriches for CIC populations that have slow growth kinetics. This enrichment may be due to selectivity of existing $\mathrm{ClCs}$ or by epigenetic reprogramming towards the CIC-state. As shown in the left side pathway, the CICs that remain following therapy play a role in disease recurrence through differentiation and subsequent proliferation. The ability of the $\mathrm{CIC}$ cells to establish heterogeneous tumors indicates a role for genetic and epigenetic reprogramming in tumor regrowth. As illustrated on the right side, therapeutic strategies to diminish CIC populations would be best delivered in combination with conventional therapies that target proliferative cells. Failure to target bulk tumor cells allows for microenvironment-induced reprogramming into $\mathrm{CIC}$ through EMT. Blocking both of these dynamic reprogramming events is a potential strategy for limiting $\mathrm{CIC}$ formation and $\mathrm{CIC}$ differentiation. Adapted from Vermeulen et al., 2012. 


\section{Epigenetics in cancer progression}

Epigenetics is the study of any heritable mechanism that influences

phenotype independent of genomic sequence (Ku et al., 2011). Some epigenetic mechanisms that impact gene expression include DNA methylation, histone modification, and microRNAs. Recent studies suggest that the pathophysiology of tumorigenesis and metastatic progression are partially dependent on epigenetic regulation as well as the accumulation of genetic mutations in oncogenes and tumor suppressors (Cieślik et al., 2013; McCleary-Wheeler et al., 2013). But unlike mutations in the DNA, epigenetic factors are malleable. Great effort has been put forth to develop small molecule inhibitors of epigenetic modulators such as histone readers, writers, and erasers.

In this dissertation, I will be focusing on histone based epigenetic mechanisms of cancer biology. Four histone proteins $(\mathrm{H} 2 \mathrm{~A}, \mathrm{H} 2 \mathrm{~B}, \mathrm{H} 3$, and $\mathrm{H} 4)$ homodimerize and complex together to form an octomeric histone. DNA wraps around the histone forming a nucleosome, and regulation of this DNA can be influenced by alterations made to the histone proteins within the nucleosome. There are a variety of histone modifications, including acetylation, methylation, sumoylation and ubiquitination (Jenuwein and Allis, 2001). Addition of these moieties typically occurs in the highly conserved, unstructured histone tails but has also been found in the more nucleosomal region of the histones. The type and location of a histone modification within the genome have been shown to determine whether or not a gene is activated or repressed (Barski et al., 2007; Creyghton et al., 2010). On top of this, different combinations of histone marks 
can affect differences in gene regulation. Observations of these phenomena have led to the histone code hypothesis, and underscore the dynamic nature of epigenetics in controlling cell fate (Strahl and Allis, 2000).

Histone modifications can correlate with cancer progression and patient prognosis (Akhtar-Zaidi et al., 2012; Verma, 2015). Loss of acetylation of lysine 16 on histone $\mathrm{H} 4$ (H4K16ac) and H4K2Ome3 is a common hallmark of cancer. Furthermore, loss of $\mathrm{H} 3 \mathrm{~K} 27 \mathrm{me} 3$ is an indicator of poor prognosis in breast, ovarian, and pancreatic cancers (Fraga et al., 2005; Gloss and Samimi, 2014; Wei et al., 2008). Song et al. showed that increased staining of H3K9Ac, H4K16Ac, H3K9me3, and H4K20me3 in NSCLC tissue samples is inversely correlated with disease recurrence and distant metastasis (Song et al., 2012). In PDAC, hyperacetylation of the Myc promoter results in transcriptional activation of the oncogene by Elk-1, a downstream target of the KRAS/MEK/ERK pathway (Köenig et al., 2010). Although not directly indicative of causation, these correlative studies suggest that the modulation of histone marks during cancer progression is of clinical relevance.

"Writers" and "erasers" are nicknames for the groups of proteins known to confer or remove histone modifications, respectively. Acetyl marks, for example, are written by histone acetyltransferases (HATs) and erased by histone deacetylases (HDACs). In fact, upregulation of HDACs in pancreatic cancer has been shown to drive tumor progression and metastasis by increasing expression of Myc and decreasing expression of epithelial marker E-cadherin (Aghdassi et al., 2012; von Burstin et al., 2009; Marshall et al., 2010; Neureiter et al., 2014). 
Histone "readers" are proteins that recognize and bind to particular histone modifications. Readers have been shown to convene an additional layer of transcriptional regulation by recruiting components of gene modulation to the chromatin. Of particular interest to my studies is histone reader BRD4, a protein that recognizes acetylated lysines.

BRD4 is associated with broad transcriptional regulation through its interactions with enhancers, and more specifically, with super-enhancers. An enhancer is a DNA element that serves as a scaffold for factors to activate transcription of a gene from a distance. This occurs regardless of the orientation of the DNA element relative to the activated gene, and can occur when the enhancer is megabases away from the activated gene (Pott and Lieb, 2015; Shlyueva et al., 2014). DNA looping is what allows enhancer elements and their associated factors to interact with and influences the transcription start sites (TSS) or promoters of target genes (Deng et al., 2012; Spitz and Furlong, 2012; Tolhuis et al., 2002).

Enhancer elements characteristically contain binding sites for multiple chromatin and transcription factors such as the p300, CBP and cohesion. Additionally, DNase I hypersensitivity experiments have shown that enhancers are typically located in areas of open chromatin (Song et al., 2012). The accumulation of certain histone modifications, H3K4me1, H3K4me2, H3K27ac, can also be used to identify enhancer sites (Barski et al., 2007; Creyghton et al., 2010; Visel et al., 2009). While it is unclear how many enhancers exist in any 
given cell type, studies based on ChIP-Seq and DNase-Seq analysis have proposed that there are upwards of 25,000 enhancer elements per cell.

A precise functional definition of a "super enhancer" has yet to be concretely determined, however, they are loosely defined as clusters of putative enhancers in close genomic proximity that are enriched for high levels of BRD4 and Mediator binding. They tend to span tens of kilobases, and have extended stretches of H3K27ac deposition (>3kb) (Heinz et al., 2015). By these parameters, most cell types are thought to have between $300-500$ superenhancers. These elements are associated with controlling numerous genes important for the regulation of cell type-specific gene expression, and consequently, cell fate (Hnisz et al., 2013; Whyte et al., 2013). For example, specific enhancers and super-enhancers have been identified that are required for the regulation of pluripotency and self-renewal. In fact, BRD4 occupies super-enhancers associated with stemness genes, and is required for transcriptional activation of those genes (Di Micco et al., 2014). It follows that super-enhancers are attractive sites for therapeutic intervention for cancer types that undergo phenotypic switching from epithelial cells into CICs during tumor progression and metastasis. By disrupting the binding of BRD4 to superenhancer elements, we expect to reduce the CIC population of a tumor and inhibit metastasis.

\section{BET Family}

BRD4 belongs to the bromodomain and extraterminal (BET) family of proteins, characterized as containing two $\mathrm{N}$-terminal bromodomains as well as an 
extra-terminal domain. The BET Family proteins BRD2, BRD3 and BRD4 are ubiquitously expressed, while BRDT is testis-specific (Jang et al., 2005).

Bromodomains (BRDs) contain a deep, largely hydrophobic acetyl-lysine binding site, and are found in 46 different proteins including HATs, HMTs, HDACs, and BETs. Broadly, BRDs have been shown to modulate enzymatic activities, protein assembly and protein-protein interactions (Ferri et al., 2016). The BRDs in BET proteins allow them to function as histone readers during transcriptional regulation. The BRDs bind to acetylated lysines residues of histone tails; these marks are typically indicative of transcriptionally active genes. The extra-terminal (ET) domain allows for interactions with other proteins including transcription factors, bringing them into close proximity of the active transcriptional machinery (Figure 3) (Shi et al., 2014).

BRD4 was first described in 1998 as a protein that interacts with the murine Mediator complex (Jiang et al., 1998). Subsequent work showed conservation of this interaction in human cells (Wu and Chiang, 2007). In 2002, mouse genetic studies demonstrated the fundamental role of BRD4 in cellular processes: BRD4 homozygous null embryos died shortly after implantation, and heterozygous mice were born with anatomical abnormalities (Houzelstein et al., 2002). It was revealed that BRD4 stimulates RNA Pol II-dependent transcription by recruiting P-TEFb (Jang et al., 2005; Yang et al., 2005). Disruption of BRD4 binding to super-enhancers results in displacement of the Mediator complex and P-TEFb from binding to super-enhancers and, ultimately, results in the loss of transcription. Since then, numerous publications have confirmed the importance 
of BET proteins in transcription and elongation. BRD2 recruits TATA box binding protein (TBP) to promoters, and has been shown to complex with TBP associated factors (TAFs), HATs, HDACs, and other chromatin remodeling factors (Denis et al., 2006; Hnilicová et al., 2013; Peng et al., 2007). On acetylated chromatin, BRD2 enhances the processivity of RNA polymerase II through gene bodies (LeRoy et al., 2008). Notably, BRD4 demonstrates the concept of epigenetic memory by remaining bound to acetylated chromatin at super-enhancers during interphase and mitosis; this facilitates initiation of postmitotic transcription (Dey et al., 2003, 2009; Hnilicová et al., 2013).

Relevant to cancer proliferation, BRD2 has been shown to regulate cell cycle by facilitating expression of cyclin D1 and p21. Similarly, BRD3 binds GATA1 to enhance expression of the transcription factor's target genes (Gamsjaeger et al., 2011; Lamonica et al., 2011). In breast cancer, GATA1 has been shown to be anti-apoptotic, proliferative, and an inducer of the EMT program (Li et al., 2015). Additionally, BRD4 has been shown to interact with oncogenic transcription factors NF-KB and TWIST to facilitate expression of their target genes (Shi et al., 2014; Zou et al., 2014). Together, the BET family of proteins has the ability to bring cancer-driving transcription factors in close proximity to the transcriptional machinery, thus potentiating expression of the cancer program. BET inhibitors are being examined as potential therapeutic interventions in the context of malignant disease. 


\section{History of BET Inhibitors in Cancer}

Some of the first bromodomain-specific inhibitors, developed in 2005 by the Zhou group, targeted bromodomain-containing proteins PCAF and CBP (Ferri et al., 2016). Triazolothienodiazepines were discovered using antiinflammatory and ApoA1 activity screens in 1990 by Mitsubishi Pharmaceuticals. JQ1, a triazolodiazepine molecule, was designed based on the Mitsubishi patent. Chromosomal rearrangements in the nuclear protein in testis gene (NUT) can result in NUT midline carcinoma (NMC), a rare subtype of head and neck cancers. Pre-clinical efficacy of JQ1 in NMC was a landmark discovery out of James Bradner's lab at the Dana Farber Cancer Institute in 2010 (Filippakopoulos et al., 2010). Simultaneously, GlaxoSmithKline (GSK) was independently developing I-BET762 to reduce inflammation in a model of sepsis (Nicodeme et al., 2010). I-BET151 is an optimized dimethylisoxazolemethypyrazole compound put forth by GSK that shows improved in vivo pharmacokinetics and terminal half-life in vivo compared to I-BET762 (Ferri et al., 2016).

Perhaps the most studied contribution of BET proteins to carcinogenesis is the role of BRD4 in transcribing proto-oncogene Myc. Numerous publications have demonstrated the therapeutic benefit of inhibiting BRD4 in the context of downregulating Myc expression. Occluding the twin bromodomains with small molecule inhibitors prevents BRD4 from binding to the enhancer region that drives expression of Myc in a variety of tumor types. This result has been shown in the following hematopoietic malignancies: multiple myeloma (MM), Burkitt's 
lymphoma (BL), acute myeloid leukemia (AML), and acute lymphoblastic leukemia (ALL) (Dawson et al., 2011; Delmore et al., 2011; Mertz et al., 2011). Abrogation of Myc expression by BET inhibition has also been shown in preclinical models of solid tumors such as NSCLC, medulloblastoma, colorectal cancer, bladder cancer, thyroid, Merkel cell carcinoma, and others (Gao et al., 2016; Sengupta et al., 2015a, 2015b; Shimamura et al., 2013; Tögel et al., 2016; Venkataraman et al., 2014; Wu et al., 2016).

BET inhibition has also shown promise in pre-clinical models via Mycindependent mechanisms. For example, FOSL1 expression is downregulated upon inhibitor treatment in pancreatic cancer and NSCLC models (Lockwood et al., 2012; Sahai et al., 2014). FOSL1 is an oncogenic transcription factor that has been shown to drive tumor progression by potentiating proliferation. In bladder cancer, BET inhibition downregulated expression of $E Z H 2$, a histone methyltransferase (HMT) that has been implicated as pathogenic in numerous tumor types (Wu et al., 2016). NF-kB is known to promote cancer survival, proliferation, and EMT in numerous malignancy types through activation of its target genes (Chen et al., 2011; Karin and Greten, 2005; Kumar et al., 2013; Min et al., 2008). BRD4 can maintain NF-kB activation by binding to acetylated lysine 310 of the p65 subunit, thus protecting it from ubiquitin-mediated degradation (Gallagher et al., 2014a). In NSCLC, JQ1 treatment impeded proliferation and resulted in proteasomal degradation of p65 (Zou et al., 2014).

An increase in transcription of pro-apoptotic protein BIM following treatment with BET inhibitors has been reported in peripheral nerve sheath 
tumors, melanoma, and thyroid cancer models (Gallagher et al., 2014b; Li et al., 2016; Patel et al., 2014). In an AML model, JQ1 treatment inhibiting binding of RNA Pol II to the promoters of $M y c$ and anti-apoptotic $B C L-2$, resulting in decreased mRNA and protein expression. At the protein level, JQ1 increased cell cycle regulator p21 and pro-apoptotic BIM. Importantly, JQ1 impeded cell division and increased apoptosis in a dose dependent manner (Fiskus et al., 2014). While the mechanism by when BET inhibition modulates expression of apoptotic proteins is unclear, BET inhibitors have been shown to increase apoptotic signaling in multiple cancer models.

While there are no BET inhibitors currently approved for therapeutic use, the competitive landscape for BET inhibitor development has undergone a global expansion over the past few years. Over a dozen different pharmaceutical companies have derived small-molecule inhibitors of BET; some have only been tested in pre-clinical studies, while others have moved onto Phase I clinical trials for hematological and solid tumors. There are currently ten active and two recently closed, but not yet published, studies testing the safety of BET inhibitors in cancer (ClinicalTrials.gov).

Merck is performing a dose-finding study with MK-8628, a promising orally available BET inhibitor that is similar in structure to JQ1. This trial recruited patients with advanced NUT midline carcinoma, triple negative breast cancer, NSCLC with KRAS mutations, castration-resistant prostate cancer, and PDAC (ClinicalTrials.gov). The study concludes in June of 2016; it will be incredibly interesting to see the results of these and other trials. Also of note is 
Resverlogix's RVX-208 compound that has shown safety in human trials for cardiovascular disease and diabetes and has advanced to several Phase II trials (Ferri et al., 2016). The SF2523 and SF2535 drugs produced by SignalRx Pharmaceuticals inhibit both BRD4 and PI3K proteins. Although still in preclinical testing, this drug may be strategically useful in the future for treatment of KRAS-mutant cancers that activate proliferative signaling through the PI3K pathway. 


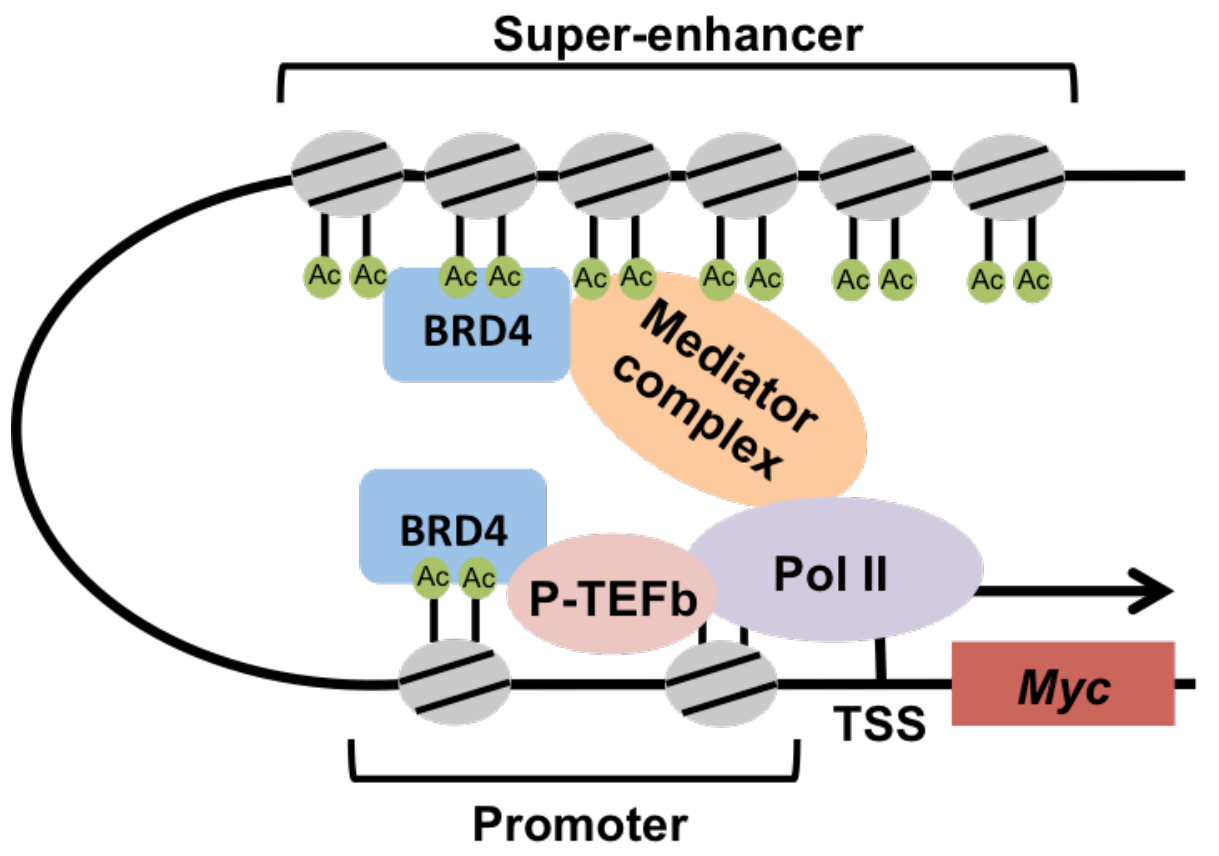

\begin{tabular}{|c|}
\hline BRD4 binding sites: \\
H4K5ac \\
H4K8ac \\
H4K12ac \\
H4K16ac \\
H3K14ac
\end{tabular}


Figure 3. Schematic of BET protein function in transcriptional activation. BET proteins bind to acetylated lysine residues on histones via their bromodomains. They are able to interact with other proteins via their extra terminal domains to facilitate recruitment of transcriptional machinery to chromatin. BRD4 has been found at both enhancer and promoter regions of genes, as depicted. BRD4 interacts with the mediator complex, which can facilitate DNA looping to bring super enhancers into proximity of promoters and transcription start sites. BRD4 has also been shown to recruit P-TEFb, an elongation factor required for the processivity of RNA Pol II. The BET family of histone readers facilitates transcriptional activation and chromatin remodeling through interactions with transcription factors, HATs, and HDACs (not depicted). Adapted from Kanno et al., 2014. 
CHAPTER 2: MATERIALS \& METHODS 


\section{Cell Culture and Inhibitor treatments}

To initiate experiments, we chose to utilize two patient-derived PDAC cell lines, 366 and 608 (Walters et al., 2013a). These cell lines were selected because they carry three predominant driver mutations in PDAC, namely in KRAS, p53 and SMAD4. 366 and 608 cells were grown in RPMI 1640 (Corning 10-040) with $10 \%$ fetal bovine serum (FBS, Invitrogen 16000044) and 5\% Penicillin/Streptomycin (Pen/Strep, Invitrogen 15070-063). Cells in this study were grown at $37^{\circ} \mathrm{C}$ and $5 \% \mathrm{CO}_{2}$. Trametinib (GSK1120212) was kindly provided by GlaxoSmithKline and used at a final concentration of $100 \mathrm{nM}$ (unless otherwise indicated). I-BET151 (GSK1210151A) was purchased from Chemietek (CT-BET151) and used at a final concentration of $10 \mu \mathrm{M}$. The A549 (NSCLC adenocarcinoma) cell line was obtained from ATCC and grown in DMEM (Corning 10-017) with 10\% fetal bovine serum (FBS, Invitrogen 16000044) and 5\% Penicillin/Streptomycin (Pen/Strep, Invitrogen 15070-063). JQ1 was gifted by Greg Wang at the University of North Carolina, Chapel Hill and used at the indicated concentrations.

\section{Three-Dimensional (3D) Multicellular Tumor Spheres}

Multicellular tumor spheres were created using a modified hanging droplet method. Cells were grown to approximately $80 \%$ confluence on standard tissueculture plates. The cells were subsequently trypsinized, resuspended in RPMI/10\% FBS/Pen/Strep, and counted. To create 25,000 cell tumor spheres, the cell suspension was diluted to $1 \times 10^{6}$ cells $/ \mathrm{mL}$, and $25 \mu$ l of the cell suspension were pipetted onto the underside of a sterile $10 \mathrm{~cm}$ Petri dish lid. 
Each lid holds approximately fifty droplets. After loading the droplets, the lid was placed onto a tissue culture plate containing $6 \mathrm{~mL}$ of sterile PBS and incubated for 48 hours to facilitate cellular aggregation and tumor sphere formation. Tumor spheres were then transferred into $10 \mathrm{~cm}$ suspension plates containing RPMI/ $2 \% \mathrm{FBS} / \mathrm{Pen} / \mathrm{Strep}$ to prevent cell attachment to the dish. Suspension plates were made by adding $8 \mathrm{~mL}$ of polyHEMA solution (Sigma-Aldrich P3932, 10 $\mathrm{mg} / \mathrm{mL}$ ) in $95 \%$ ethanol to sterile polystyrene Petri dish plates (Fisher Scientific). The plates were then incubated for 24 hours in a sterile environment to allow the ethanol to evaporate. Prior to use, plates were washed with sterile PBS to remove any residual ethanol or other contaminants. Each suspension plate holds up to 100 tumor spheres. To induce EMT, tumor spheres were incubated with recombinant cytokines TNF (Invitrogen PHC3016, $10 \mathrm{ng} / \mathrm{mL}$ ) and TGF $\beta$ (Invitrogen PHG9024, 2 ng/mL).

\section{Tumor sphere Replating Assay}

To assess cell count of tumor spheres treated with inhibitors, tumor spheres were collected and centrifuged at $1000 \times \mathrm{g}$ for 5 minutes. Media was aspirated and the tumor spheres were washed twice with $5 \mathrm{~mL}$ PBS before incubating in $0.5 \mathrm{~mL}$ of $0.05 \%$ trypsin in EDTA for 5 minutes at $37^{\circ} \mathrm{C}$. Tumor spheres were dissociated by gentle trituration and replated onto $60 \mathrm{~mm}$ tissue culture dishes in RPMI with 10\% FBS and Pen/Strep. Cells were given between 12 and 24 hours to attach to the tissue culture dish before being washed with PBS twice and further dissociated by $1 \mathrm{~mL}$ of trypsin. The cells were then counted using a TC10 Automated Cell Counter (Bio-Rad 1450016). Cell counts 
from inhibitor treated conditions were measured relative to the DMSO vehicle condition.

\section{Scratch Assay}

Cells were plated in $60 \mathrm{~mm}$ tissue cultures dishes and grown to confluency in complete media. Using a pipette tip, two scratches were made through the plate, perpendicular to the marker lines. The cells were washed with PBS to remove cells displaced during scratching, RPMI media was replaced with 2\% FBS + Pen/Strep and inhibitors were added (50 nM trametinib, $10 \mu \mathrm{M} \mathrm{I-}$ BET151). Pictures of the scratch widths were taken at the intersection of the scratch and the two marker lines. Images were obtained at the indicated time points after the scratches were drawn. TScratch software was used according to instructions to quantify the invaded area of each scratch region. The $\%$ invaded area for each treated condition was compared relative to the size of the original scratch for each condition.

\section{Caspase 3 Activity Assay}

Caspase 3 activity was measured in whole cell lysates according to the protocol provided in the Genetex Caspase-3 Colorimetric Assay Kit (Genetex GTX85558). Briefly, tumor spheres were treated with a DMSO vehicle control or alone or in combination with trametinib and I-BET151 for 48 hours before being collected by centrifugation at $1000 \mathrm{xg}$ for 5 minutes. The tumor spheres were washed in PBS twice before being resuspended in the provided cell lysis buffer and incubated on ice for 10 minutes. The lysed cells were centrifuged at 10,000 $\mathrm{x} \mathrm{g}$ and the supernatants collected and assayed for protein concentration using a 
Bradford assay protocol. Equal amounts of protein were diluted to equal volume in buffer before being incubated with $p$-nitroanilide (pNA) conjugated substrates for 2 hours on ice. Active caspase 3 will cleave the substrate thus releasing pNA, which can be detected using a Molecular Devices SpectraMax M2 Microplate Reader set to an absorbance of $405 \mathrm{~nm}$. Absorbance levels were normalized to a blank sample and inhibitor treatment conditions were compared relative to the DMSO vehicle control condition.

\section{Orthotopic Xenograft Tumor Model and Histopathology}

Experiments were carried out by Sara Adair in the Parsons Lab at the University of Virginia. Human tumor explants ( $\sim 50 \mathrm{mg})$ were surgically sewn onto the pancreata of 6- to 8-week-old male athymic nude mice to establish the orthotopic xenograft model. Tumors were allowed to grow for 3 to 4 weeks to a volume of 100 to $500 \mathrm{~mm}^{3}$ as assessed by volumetric magnetic resonance imaging (MRI), at which point drug treatment commenced. Mice were treated with either vehicle $(0.5 \%$ hydroxypropyl as indicated in the text and figures. Dosing was given as follows: $1 \mathrm{mg} / \mathrm{kg}$ trametinib and $25 \mathrm{mg} / \mathrm{kg}$ I-BET151 - both drugs were administered once daily by oral gavage).

In all experiments, volumetric MRI was used to assess changes in tumor volume at 7-day intervals while on drug treatment, as previously described (Lindberg et al., 2014; Walters et al., 2013a). Mice were then sacrificed, tumors were excised, weighed, and measured by calipers, and mice were examined for the presence of metastatic disease. To determine the therapeutic efficacy of drug combinations, MRIs were obtained at the start of treatment to establish an initial 
tumor volume baseline for each mouse. Subsequent interval MRI studies were used to assess the change in tumor volume in response to treatment. The interval tumor volumes were compared relative to the initial tumor volume to calculate the relative change in tumor volume (fold change) for each tumor. Linear regression was used to model a line of best fit for the tumor fold change data plotted relative to time. The slope of that line of best fit served as an estimate of the tumor growth rate for each treatment group expressed as the fold change per week on treatment. Upon completion of the experiment, tumor samples were placed in Allprotect (Qiagen, Valencia, CA), snap-frozen in liquid nitrogen, or fixed in formalin for histologic analysis.

This study was carried out in strict accordance with recommendation from the Animal Care and Use Committee (ACUC) of the University of Virginia. The protocol was approved by ACUC Number 4078. All experiments were terminated 8 weeks post implantation at which time tumors were less than $1.5 \mathrm{~cm}^{3}$ in size, restricting tumor burden. All efforts were made to minimize pain and suffering.

\section{Flow Cytometry}

These experiments were performed by Sara Adair in the Parsons Lab, University of Virginia. Tumor spheres were harvested by centrifugation at 1000 g, washed gently with PBS and dissociated using TrypLE (Invitrogen, Carlsbad, CA) for 5 minutes at $37^{\circ} \mathrm{C}$. Tumor spheres were then mechanically disrupted using a $200 \mu \mathrm{L}$ pipet. A single cell suspension was achieved using a $70 \mu \mathrm{m}$ filter. Cells were maintained on ice throughout staining procedure. Samples were stained using CD44 FITC, CD24 PE-CF594 (Becton Dickenson, Franklin Lakes, 
NJ), and EpCAM (Miltenyi Biotec, Bergisch Gladbach, Germany). DAPI (SigmaAldrich, Saint Louis, MO) served as a viability marker. OneComp ebeads were used for controls (eBioscience, San Diego, CA). Sphero AccuCount Particles (Spherotech, Lake Forest, IL) were used to obtain equal cell numbers for cell cycle analysis. After stem cell data were collected, cells were fixed using $1.5 \%$ paraformaldehyde for 10 minutes at room temperature and stored in PBS at $4 \mathrm{C}$. Nuclear staining for cell cycle was achieved using DAPI (Sigma-Aldrich) at 1 $\mu \mathrm{g} / \mathrm{mL}$ after permeabilization of fixed cells using $100 \%$ ice cold methanol for 10 minutes. Data were collected using a BD FACS Calibur (Becton Dickenson) in collaboration with the University of Virginia Flow Cytometry Core Facility. Data were analyzed using FlowJo, version 9.0 (TreeStar, Ashland, OR).

\section{Quantitative Real-Time Polymerase Chain Reaction (QRT-PCR)}

Total RNA was isolated according to protocol using the Zymo Research Quick-RNA MiniPrep (Plus) kit (Zymo Research R1058) and cDNA was synthesized by reverse transcription according to protocol using the Bioline SensiFAST cDNA Synthesis Kit (Bioline BIO-65054). Relative expression of mRNA was evaluated by quantifying cDNA samples with gene-specific primer sets by QRT-PCR using a SYBR Green/Taq Supermix (Bio-Rad 170-8884). Results were normalized to GAPDH. QRT-PCR primers are listed in Table 1. 
Table 2: qRT-PCR PRIMERS

\begin{tabular}{|c|c|}
\hline Gene & Sequence \\
\hline H. sapiens GAPDH F & GAAGGTGAAGGTCGGAGTC \\
\hline H. sapiens GAPDH R & GAAGATGGTGATGGGATTTC \\
\hline H. sapiens KLF4 F & ACCAGGCACTACCGTAAACACA \\
\hline H. sapiens KLF4 R & GGTCCGACCTGGAAAATGCT \\
\hline H. sapiens MMP2 F & TCTCCTGACATTGACCTTGGC \\
\hline H. sapiens MMP2 R & CAAGGTGCTGGCTGAGTAGATC \\
\hline H. sapiens MMP9 F & TTGACAGCGACAAGAAGTGG \\
\hline H. sapiens MMP9 R & GCCATTCACGTCGTCCTTAT \\
\hline H. sapiens POU5F1 F & ACCCCTGGTGCCGTGAAGC \\
\hline H. sapiens POU5F1 R & CAGATGGTCGTTTGGCTGAATACC \\
\hline H. sapiens SNAl1 F & CACTATGCCGCGCTCTTTC \\
\hline H. sapiens SNAl1 R & GGTCGTAGGGCTGCTGGAA \\
\hline H. sapiens SNAI2 F & ATGAGGAATCTGGCTGCTGT \\
\hline H. sapiens SNAI2 R & CAGGAGAAAATGCCTTTGGA \\
\hline H. sapiens SOX2 F & GCCCAGGAGAACCCCAAGATG \\
\hline H. sapiens SOX2 R & GCTCGCAGCCGCTTAGCCTC \\
\hline H. sapiens TWIST1 F & CGGGAGTCCGCAGTCTTA \\
\hline H. sapiens TWIST1 $\mathrm{R}$ & CTTGAGGGTCTGAATCTTGCT \\
\hline H. sapiens VIM F & CTTCGTGAATACCAAGACCTGC \\
\hline H. sapiens VIM R & TATCAACCAGAGGGAGTGAATCC \\
\hline H. sapiens ZEB2 F & CAATACCGTCATCCTCAGCA \\
\hline H. sapiens ZEB2 $\mathrm{R}$ & CCAATCCCAGGAGGAAAAAC \\
\hline H. sapiens NANOG F & CATGAGTGTGGATCCAGCTTG \\
\hline H. sapiens NANOG $R$ & CCTGAATAAGCAGATCCATGG \\
\hline H. sapiens BIM-all F & GCGCCCAGAGATATGGATCGC \\
\hline H. sapiens BIM-all $R$ & TGGGTGGTCTTCGGCTGCT \\
\hline H. sapiens $B I M_{E L} F$ & ACGGAGGTGAAGGGGACAGC \\
\hline H. sapiens BIMEL $R$ & AGCAAAAGGGCCAGGGCTG \\
\hline H. sapiens $B I M_{L} F$ & GGCСССTACCTCCCTACAGA \\
\hline H. sapiens $B I M_{L} R$ & GCCGCAACTCTTGGGCGAT \\
\hline H. sapiens BIMS F & GAGCCACAAGCTTCCATGAG \\
\hline$H$. sapiens BIMS $R$ & TAACCATTCGTGGGTGGTCT \\
\hline H. sapiens $A B C B 1 \mathrm{~F}$ & TGACATTTATTCAAAGTTAAAAGCA \\
\hline H. sapiens $A B C B 1 R$ & TAGACACTTTATGCAAACATTTCAA \\
\hline H. sapiens $A B C C 1 \mathrm{~F}$ & AGTGGAACCCCTCTCTGTTTAAG \\
\hline H. sapiens $A B C C 1 R$ & CCTGATACGTCTTGGTCTTCATC \\
\hline H. sapiens $A B C G 2 F$ & CCGCGACAGTTTCCAATGACCT \\
\hline H. sapiens $A B C G 2 R$ & GCCGAAGAGCTGCTGAGAACTGTA \\
\hline
\end{tabular}

F, forward primer; R, reverse primer. All sequences listed from 5'-3'. 


\section{Immunoblotting and Antibodies}

Proteins in cell lysates were measured using a Bradford Assay. An equal amount of protein per sample (>15 $\mu \mathrm{g})$ was prepared in NuPAGE LDS $4 \mathrm{X}$ Sample Buffer (Invitrogen NP0007) and run alongside a protein ladder (Bio-Rad 1610374). Proteins were separated on $4-12 \%$ Bis-Tris polyacrylamide gel electrophoresis (PAGE) gels in SDS buffer for 90 minutes at 150 volts. Protein was transferred from the gel onto nitrocellulose membranes from 60 minutes at 40 volts. Membranes were blocked for one hour at room temperature in $5 \%$ milk in TBS-T. Primary antibodies used for immunoblot are E-cadherin (BD Pharmingen 610404), Vimentin (Sigma F1804), $\alpha$-tubulin (Sigma T6793), GAPDH (GeneTex GTX627408), BIM (Cell Signaling 2819), Acetylated Histone 3-K9/K14 (Upstate 06-599), Hyperacetylated Histone 4-K5/K8/K12/K16 (Upstate 06-946), Acetylated tubulin (Sigma T 6793), AIF (Cell Signaling 4642), BAK (Upstate 06-536), BAX (Santa Cruz sc-493), Cleaved-Caspase 3 (Cell Signaling 9661), ERK (Cell Signaling 9102), LC3A/B (Cell Signaling 4108), Phospho-BIM (Cell Signaling 4581), Phospho-ERK (Cell Signaling 9101), Pro-Caspase 3 (Santa Cruz sc-7148), Puma (Cell Signaling 4976), $\beta$-actin (Sigma A 5316). Secondary antibodies with enzyme conjugates to horseradish peroxidase were used (Mouse IgG HRP Conjugate, Promega PR-W4021 and Rabbit IgG HRP Conjugate, Promega PR-W4011). Chemiluminescence reagents (Luminata Classico Western HRP Substrate, Millipore WBLUC0500) were used to expose autoradiography film (HyBlot CL, Denville E3018). 


\section{Mitochondrial Fractionation}

Tumor spheres were collected by centrifugation at $750 \mathrm{~g}$ for 5 minutes at $4^{\circ} \mathrm{C}$, washed with 2 pellet volumes of PBS, centrifuged again and the PBS was aspirated. The pellet was resuspended in 1 pellet volume of hypotonic lysis buffer $(10 \mathrm{mM} \mathrm{KCl}, 1.5 \mathrm{mM} \mathrm{MgCl} 2,1 \mathrm{mM}$ EDTA, $1 \mathrm{mM}$ EGTA, and $250 \mathrm{mM}$ Sucrose) and dounced using roughly 100 strokes in a $1 \mathrm{~mL}$ Teflon homogenizer. The homogenate was transferred to an eppendorf tube and centrifuged twice at $750 \mathrm{~g}$ for 10 minutes at $4^{\circ} \mathrm{C}$, transferring the supernatant to a new tube after each spin; the pellet containing the nuclear fraction of the cells was frozen or lysed in high salt RIPA buffer for immunoblot. The supernatant from the previous step was then centrifuged again at $10,000 \times \mathrm{g}$ for 10 minutes at $4^{\circ} \mathrm{C}-$ the resultant supernatant contains the cytoplasmic extract while the pellet contained the mitochondrial fraction. The mitochondrial pellet was lysed in $25 \mu \mathrm{L}$ of RIPA buffer and incubated on ice for 30 minutes before being centrifuged at $10,000 \times \mathrm{g}$ for 10 minutes at $4^{\circ} \mathrm{C}$ and the supernatant collected. Protein levels for all cell fractions were measured using a Bradford protein assay.

\section{Transwell Assays}

Tumor spheres were disaggregated by incubation with $0.05 \%$ trypsin. Tumor spheres were also subjected to intermittent gentle mechanical disruption with a pipette to facilitate disaggregation. Transwell assays were performed to measure migration and invasion according to the manufacturer's protocol (BD Biosciences). $1 \times 10^{5}$ cells (migration) or $5 \times 10^{4}$ cells (invasion) were seeded in plain RPMI in the top chamber of a transwell control plate (BD 354578) or 
Matrigel invasion plate (BD 354480). RPMI containing 10\% FBS and was loaded into the bottom well the plates which were then incubated for 8 hours (migration) or 24 hours (invasion) at $37^{\circ} \mathrm{C}$ and $5 \% \mathrm{CO}_{2}$. After incubation, the non-migrating or non-invading cells on the upper side of the membrane were removed by scrubbing. The remaining cells were fixed in $100 \%$ methanol at $-20^{\circ} \mathrm{C}$ for 1 hour and stained with $0.1 \%$ crystal violet at ambient temperature for 1 hour. The stained cells were imaged and quantified using Adobe ${ }^{\circledR}$ Photoshop.

\section{Statistics}

All data are presented as mean \pm SD of three independent biological experiments. A one-tailed Student's $t$ test in Microsoft Excel was performed to compare two experimental conditions. Differences were considered statistically significant when indicated by $p \leq 0.05$ (indicated by *), $p \leq 0.01{ }^{* *}$ ), or $p \leq 0.005$ $\left({ }^{* * *}\right)$. 
CHAPTER 3: TRAMETINIB AND I-BET151 AS A NOVEL COMBINATORIAL THERAPY FOR PANCREATIC ADENOCARCINOMA 


\section{INTRODUCTION}

\section{Pancreatic Cancer}

Pancreatic cancer has the shortest survival duration of any solid organ malignancy, with a median survival of only 6 months post diagnosis (Chang et al., 2014; Chiorean and Coveler, 2015). The 5-year survival rate remains around $6 \%$, a number that has barely budged over the last 40 years despite ongoing cancer research efforts (Chand et al., 2016). In the US, carcinoma of the pancreas is the $10^{\text {th }}$ leading cause of cancer, however it is the $4^{\text {th }}$ leading cause of cancer related deaths. According to the American Cancer Society, 2016 will see an estimated 48,960 new diagnoses and 40,560 deaths due to pancreatic cancer (American Cancer Society, 2016). It is hypothesized that the numbers of patients diagnosed with this disease will double by 2050 as the result of an aging population and the increase in US obesity rates, a known risk factor for pancreatic cancer (Rahib et al., 2014; Silverman et al., 1999). These bleak statistics underscore the need to propose and evaluate more effective therapeutic strategies against this disease.

Contributing to the poor survivability of pancreatic tumors is the fact that they go undiagnosed during early stages of the disease; the patient is often asymptomatic when the cancer is in a localized area of the pancreas. Even when patients do show symptoms such as jaundice, fatigue, abdominal pain, or unexplained weight loss, they can often be attributed to a number of pathologies or ailments. The anatomical location of the pancreas makes it difficult to image small masses, further complicating and delaying diagnosis. Ongoing studies are 
attempting to elucidate serum-based biomarkers such as autoantibodies, signaling or enzymatic proteins, and non-coding RNAs with the hopes of facilitating the early diagnosis of this malignancy (Dimastromatteo et al., 2015; Kelly et al., 2015).

Pancreatic cancer can be divided into two subtypes: exocrine and neuroendocrine tumors (NETs). Roughly $95 \%$ of pancreatic cancers are exocrine adenocarcinomas arising from the epithelial cells of the ductal tissue. Pancreatic ductal adenocarcinomas (PDAC) make up roughly $90 \%$ of all pancreatic cancer diagnoses (Chiorean and Coveler, 2015). The pancreas is a gland that produces hormones and enzymes that are secreted to the duodenum through a tree-like structure of ducts. Although the ducts only make up $10 \%$ of the overall pancreatic tissue, they are the primary tissue type in which tumors develop. Additionally, tumors are most commonly located near the head of the pancreas, making surgery precarious due to the proximity to a number of vital vascular structures (Hussain, 2016).

Thus far, the most successful treatment option is surgical resection of the tumor or a pancreatectomy, however, this is only available to the $20 \%$ of pancreatic patients who present with a localized disease (Sinn et al., 2016). Despite surgery, these patients have a 5 -year survival rate of only $20 \%$ due to recurrent or metastatic disease (Dimastromatteo et al., 2015). The majority of patients are diagnosed with unresectable tumors and lymph node involvement, which leaves no viable treatment options. Pancreatic cancers are generally 
resistant to conventional cancer therapeutics such as chemotherapy and radiation.

All together, the unfortunate outcome of this disease only leaves room for improvement in terms of therapeutics. Ongoing studies are underway to better understand the genetic complexity, molecular biology and signaling pathways of PDAC. Scientists are delving into the role of cancer stem cells, epigenetics, tumor microenvironment, evasion of the immune system, and cancer metabolism to elucidate and exploit the vulnerabilities of these tumors. Models of precursor lesions, such as acinar-ductal metaplasia (ADM) and pancreatic intraepithelial neoplasias (PanINs), are revealing answers about how this disease begins and progresses towards metastatic disease (Grippo et al., 2003). Novel animal models are available to test the effects of new treatments (Walters et al., 2013a). Clinical trials are testing more targeted approaches such as small molecule inhibitors and immunotherapies.

\section{Apoptotic resistance in PDAC}

A major cause of therapeutic failure against PDAC is the propensity of these tumors to evade apoptosis (Westphal and Kalthoff, 2003). Apoptosis, also known as programmed cell death, is a cellular program that regulates normal tissue homeostasis. The apoptotic machinery removes redundant, damaged or infected cells, and is a fundamental process in protecting against neoplastic growth. Interestingly, the fraction of apoptotic cells in a PDAC tumor can predict overall survival of a patient: a high apoptotic index correlates with a more favorable prognosis than a low apoptotic index (Arlt et al., 2013; Trauzold et al., 
2003). Likewise, increased expression of anti-apoptotic proteins such as BCL-2, $B C L-x L$ and survivin have been shown to correlate with poor prognosis, thus highlighting the importance and contribution of apoptosis to the tumor biology of PDAC (Fulda, 2009; Hamacher et al., 2008). Repressing survival signals and activating pro-death signals in cancer cells would provide considerable help in combating chemo- or radioresistance.

There are two pathways to facilitate apoptosis: the intrinsic and the extrinsic pathways. Both pathways result in the activation of "effector" or "executioner" caspases 3,6, and 7. Caspase proteins are cysteine proteases that exist as "pro" forms and are stimulated by cleavage next to aspartate residues. Cleavage of pro-caspases results in the caspase cascade, or a series of protease activity to cleave substrates. "Activator" caspases 8 and 9 will be triggered depending on whether the intrinsic or extrinsic apoptotic pathway is induced, respectively. The "activator" caspases, in turn, cleave the "effector" caspases that become activated to enzymatically degrade substrates including nuclear lamins, DNase inhibitors, cytoskeletal proteins, and other caspase molecules. The result of triggering the effector caspases includes a commitment to apoptosis, as well as the morphological alterations that result in cell death (Fulda, 2009; Mukhopadhyay et al., 2014; Westphal and Kalthoff, 2003).

The extrinsic or death receptor apoptotic pathway is activated by ligands such as Fas ligand (FasL), tumor necrosis factor (TNF), TNF-related apoptosis inducing ligand (TRAIL), and TNF-related weak inducer of apoptosis (TWEAK). These signals originating from outside the cells bind to death receptors in the cell 
membrane that harbor extracellular domains such as Fas receptor (FasR), tumor necrosis factor receptor 1 (TNFR1), and the death receptors 3 and 4 (DR3 and DR4). Upon ligand binding, the receptors will oligomerize, recruit adaptor proteins to activate caspase- 8 to propagate the apoptosis signal by cleaving caspase-3 (Westphal and Kalthoff, 2003). PDAC cells appear to overcome extrinsic apoptosis mechanisms through downregulation or mutation of the apoptotic machinery, or overexpression of anti-apoptotic proteins (Trauzold et al., 2003). For example, pancreatic cancer cell lines have been shown to produce high levels of FAP-1, a phosphatase that inhibits the apoptotic function of Fas (Ungefroren et al., 1998).

Intrinsic apoptosis can be triggered by a number of stimuli including cytotoxic agents, growth factor deprivation, hypoxia, and cellular stress. Under this mechanism of apoptosis, mitochondrial integrity is maintained by the balance of pro- and anti-apoptotic proteins in the B-cell CLL/lymphoma 2 (BCL-2) family of proteins (Modi et al., 2015; Sionov et al., 2015). Pro-apoptotic proteins include BIM, BAX, BAK, BID, BAD, NOXA, and PUMA, while the anti-apoptotic proteins are BCL-xL, BCL-2, MCL1, and A1 (Correia et al., 2015). BCL-2-Interacting Mediator of cell death (BIM) is a direct activator of apoptosis that encourages oligomerization of BCL-2-associated X protein (BAX) and BCL-2 Homologous Antagonist Killer (BAK) to initiate permeabilization of the mitochondrial outer membrane (MOM). Intrinsic apoptosis is diagramed in Figure 4.

Cancer cells have adapted mechanisms to abrogate mitochondrial apoptosis. For example, cells harboring a constitutively activating KRAS 
mutation have increased levels of phosphorylated ERK1/2. ERK-dependent phosphorylation of BIM is recognized by several E3 ligases. These E3 ligases the recruit $\mathrm{E} 1$ and $\mathrm{E} 2$ to stimulate polyubiquitination of $\mathrm{BIM}$, which is then targeted for degradation through the $26 \mathrm{~S}$ proteasome (Ewings et al., 2007). Furthermore, cells containing mutations in tumor suppressor p53 fail to induce apoptosis via p53 upregulated mediator of apoptosis (PUMA) (Harada and Grant, 2012). My study proposes the use of inhibitors to increase and stabilize cellular levels of BIM to allow for apoptosis in cancer cells.

Transcription of BIM is suppressed by growth factors and cytokines. Cessation of these factors results in induction of BIM by the transcription factor FOXO3a. In a KRAS mutant background, FOXO3a is sequestered in the cytosol via AKT-mediated phosphorylation, a pathway downstream of KRAS. Additional transcription factors that can mediate BIM transcription include c-Jun, CHOP-C (activated by ER stress signals), RUNX3 (responsible for TGFB-induced apoptosis), and E2F1 (Puthalakath et al., 2007; Yano et al., 2006). Modulation of BIM expression is also controlled through epigenetic mechanisms (RichterLarrea et al., 2010). Deacetylation of the BIM promoter can also repress activation of $B I M$ transcription; treatment with an HDAC inhibitor combats $B I M$ repression and contributes to cell death in ALL (Bachmann et al., 2010). HDAC inhibitors have also been shown to recruit E2F1 to the BIM promoter (Zhao et al., 2005). Furthermore, the BIM promoter can be hypermethylated to repress expression, and treatment with 5-aza-2-deoxycytidine can restore expression of BIM in CML cells (San José-Eneriz et al., 2009). 
Splicing factors, such as SRp55 (SRSF6), can act on nascent BIM mRNA to produce the various isoforms of $B I M$ : $B I M_{E L}, B I M_{L}$ and $B I M_{S}$. Splice variation can alter the ability of a BIM isoform to potentiate apoptotic signaling in two ways. Firstly, $B I M_{E L}$ and $B I M_{L}$ can be sequestered to the cytoskeleton via their dynein light chain I binding sites. In contrast to $B I M_{E L}$ and $B I M_{L}, B I M_{S}$, lacks this binding site and cannot be sequestered on the microtubule complex and, therefore, is free to activate apoptosis upon production (Hara et al., 2013). Secondly, BIMEL is the only splice variant that can be phosphorylated by ERK for ubiquitinmediated degradation. Downregulation or inhibition of SRp55 leads to increased production of the splice variant $\mathrm{BIM}_{\mathrm{S}}$, the most potent activator of cell death of all the BIM splice variants (Hara et al., 2013; Juan et al., 2014). Downregulation or loss of Gli-similar 3 (GLIS3), a zinc finger transcription factor, also downregulates SRp55 - this leads to increased production of the BIMs splice variant (Nogueira et al., 2013). 


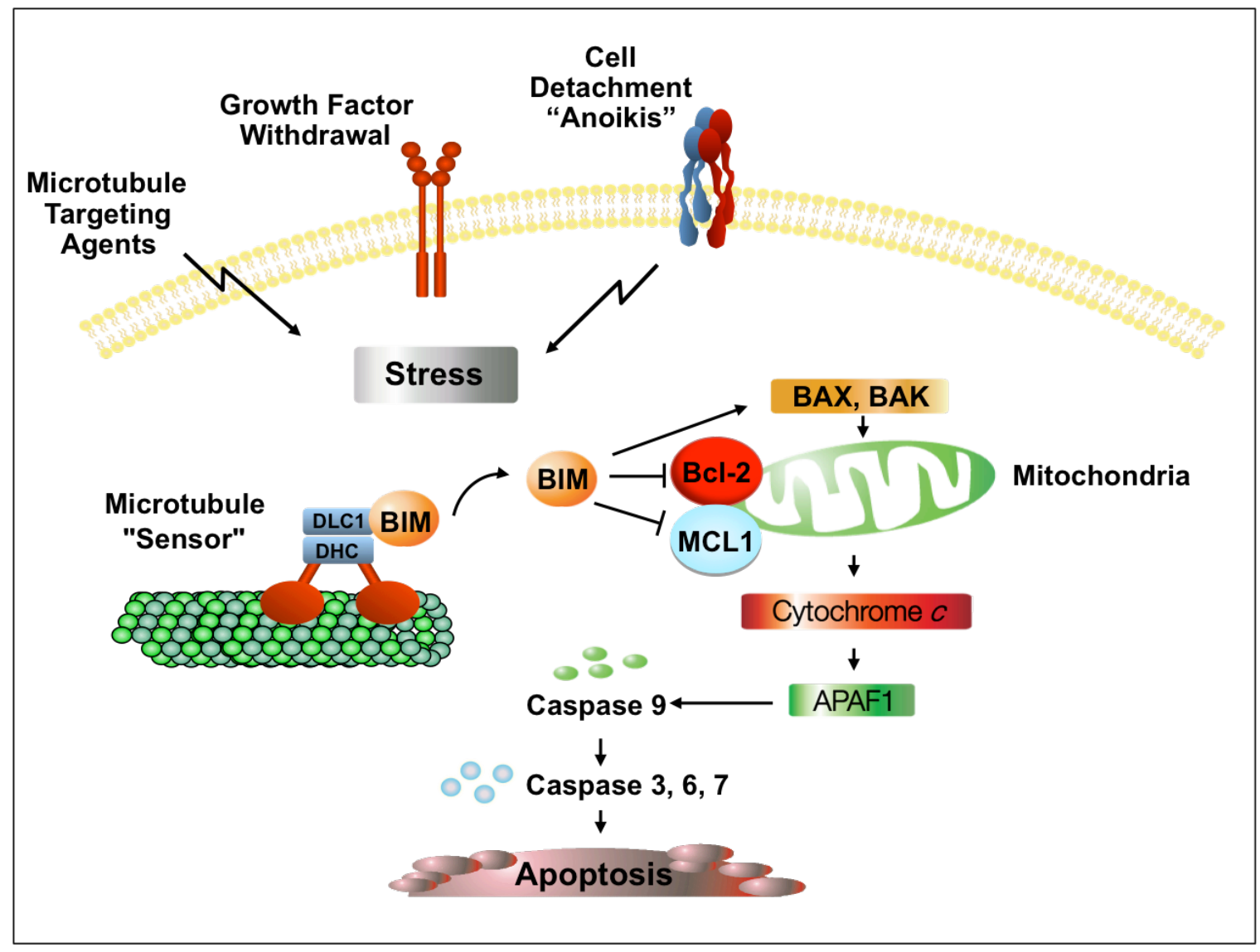


Figure 4. BIM-Induced Apoptosis. Under conditions of cellular stress, BIM is released from sequestration on the microtubules and translocates to the mitochondria. BIM interacts with anti-apoptotic proteins such as BCL-2 and MCL1 to disrupt their inhibition of BAX and BAK. Freed BAX and BAK associate with the mitochondrial outer membrane (MOM) and oligomerize to form a channel or pore in the MOM; this results in the release of cytochrome $\mathrm{C}$ into the cytosol. Cytochrome $\mathrm{C}$ activates APAF1, which in turn activates caspase 9, the initiator caspase enzyme. Caspase 9 cleaves the effector caspases 3, 6 and 7, thus activating their proteolytic degradation that leads to cellular destruction. 


\section{Oncogenic KRAS signaling in PDAC}

Kirsten rat sarcoma viral oncogene homolog (KRAS) is mutationally activated in $75-95 \%$ of PDAC cases. Found in low-grade PanIN lesions, KRAS mutations are hypothesized to be one of the earliest genetic alterations that give rise to PDAC (Eser et al., 2014; Kanda et al., 2013; Morris et al., 2010). The point mutation at codon 12 from a glycine to an aspartic acid $\left(K R A S^{G 12 D}\right)$ or to valine $\left(K R A S^{G 12 V}\right)$ results in constitutive activation of the KRAS GTPase. While G12D and G12V are commonly known gain-of-function mutations in KRAS, other mutations in codon 12 are observed in human PDAC. These persistently active forms of KRAS drives signaling pathways that contribute to sustained proliferation, apoptotic evasion, cell migration, and metabolic reprogramming (Blum and Kloog, 2014; Cohen et al., 2015; Kranenburg, 2005; Ryan et al., 2014). Murine models have shown that tissue specific KRAS mutations are sufficient to generate ADM and PanIns that have the ability, after a long latency period, to develop into invasive metastatic PDAC (Grippo et al., 2003; Guerra et al., 2007; Hingorani et al., 2005). Inactivating mutations to tumor suppressor genes CDKN2A, TP53, and SMAD4 accelerate PDAC progression in mice; these genes are also mutated at high frequency in patient populations (McCubrey et al., 2007).

The KRAS gene encodes a $21 \mathrm{kDa}$ protein with GTPase function. Under normal physiological conditions, when a growth factor such as EGF or TGF binds to its tyrosine kinase receptor (RTK), the receptor will dimerize, activate, and transphosphorylate. This recruits guanine nucleotide exchange factors (GEFs) to 
replace an inactivating GDP moiety with an activating GTP moiety on KRAS. Activated KRAS then binds Raf or PI3K to trigger downstream kinase cascade pathways. Raf is a serine/threonine kinase that phosphorylates MEK1 and MEK2 (MAP2K1 and MAP2K2, respectively). MEK1/2 then phosphorylates ERK1 and ERK2 (MAPK3 and MAPK1, respectively) to promote transcription of target genes that regulate cell cycle, survival and metabolism. This is known as the MAPK/ERK or Ras-Raf-MEK-ERK pathway. Similar phenotypes result from stimulating the PI3K/PDK1/AKT/mTOR pathway. Feedback loops and cross-talk occurs between the MEK1/2 pathway and PI3K-AKT-mTOR. Direct targeting of mutant KRAS in cancer has largely failed due to the ability of PDAC to survive by activating alternate proliferative pathways (Eser et al., 2014; McCubrey et al., 2007; Ryan et al., 2014).

MEK1 and MEK2 are members of the mitogen-activated protein kinase kinase family that can phosphorylate tyrosine, serine and threonine residues on ERK1 and ERK2; this results in the translocation of ERK to the nucleus (Crews et al., 1992). In oncology, it is rare to find de novo mutations in MEK itself, however, its activity is required for tumorigenesis due to mutations occurring in genes upstream of MEK (e.g. KRAS or BRAF). ERK is a key regulator of cellular activity due to its ability to phosphorylate over 600 targets including transcription factors ELK1, Fos, Jun, and Myc, known oncoproteins that contribute to cancer survival, proliferation and maintenance (McCleary-Wheeler et al., 2013; McCubrey et al., 2007). Feedback loops and cross-talk exist between the MEK pathway and the PI3K-AKT-mTOR, JNK-MAPK, NF-kB, Wnt-B-catenin, 
Hedgehog, Notch, TGFß-SMAD pathways (Neuzillet et al., 2014; Normanno et al., 2006). Aberrant activation of one or more of these pathways can result in an autocrine and/or paracrine loop that potentiates proliferation and survival.

Of interest to this study, the MEK/ERK pathway has the ability to inhibit apoptotic signaling. Phosphorylation by ERK can result in the degradation of pro-apoptotic proteins including BIMEL and caspase 9 (Allan et al., 2003; Neuzillet et al., 2014). In certain contexts, downstream signaling of ERK can repress expression of apoptotic gene $B I M$ and $B A D$, while inducing expression of survival genes that produce BCL-2, BCL-xL and MCL1 (Arlt et al., 2013). In BRAF mutant melanoma, apoptotic resistance to the selective BRAF inhibitor PLX4720 correlated with increases in the activation of ERK (Lai et al., 2012). This pathway also contributes to mitochondrial fragmentation, which disallows apoptosis by preventing BAX from docking on the surface of the mitochondria (Renault et al., 2015).

Trametinib (Mekinist, GlaxoSmithKline) is a small-molecule inhibitor of MEK1 and MEK2 that was discovered through a high-throughput screening method. In 2013, trametinib was FDA approved as a monotherapy for the treatment of unresectable or metastatic BRAF mutant malignant melanoma (Wright and McCormack, 2013). The orally available compound specifically binds to MEK1 and MEK2 to prevent downstream activation of ERK, and has been shown to block growth factor-mediated proliferation in melanoma. It is currently being tested for efficacy in NSCLC, refractory leukemia, and PDAC. Clinical trials measuring drug safety have shown that trametinib is tolerable alone 
or in combination with gemcitabine (Infante et al., 2012, 2013). Following these results, a randomized, double-blind, placebo-controlled trial testing the efficacy of trametinib in combination with gemcitabine in patients with metastatic PDAC was conducted. The study showed that the combination therapy was not significantly different in prolonging overall survival or progression free survival compared to gemcitabine alone (Infante et al., 2014).

\section{A Novel Combinatorial Therapy for PDAC: trametinib and I-BET151}

In this study, we examine the efficacy of a combinatorial therapy that counters several characteristics of the hallmarks of cancer: proliferation and apoptotic evasion. Trametinib inhibits downstream signaling of oncogenic KRAS through the MAPK pathway to impede proliferative and survival signals. To further promote apoptosis, cellular levels of BIM can be increased using IBET151. Navitoclax, a BCL-2/BCL-xL antagonist, synergizes with MEK inhibitor G-963 to induce apoptosis (Tan et al., 2013). We predict that increasing cellular levels of BIM will have similar effects by blocking the pro-survival mechanism of $B C L-2$ and $B C L-x L$. BET inhibition induces the expression of $B I M$ in $B R A F$ mutant melanoma (Gallagher et al., 2014b), and we anticipate it will do the same in KRAS mutant PDAC.

Additionally, we postulated that the addition of I-BET151 to trametinib would diminish the population of cells that are refractory to trametinib by preventing transcriptional reprogramming of cells through ERK-independent genes to facilitate survival (Fig. 5). Cancer cells have been shown to escape adjuvant therapy through the induction of EMT and CIC phenotypes. I-BET151 is believed 
to disrupt super-enhancers necessary for phenotypic switching. We predict that I-BET151 will block upregulation of EMT and CIC associated genes in response to trametinib treatment. 
HYPOTHESIS

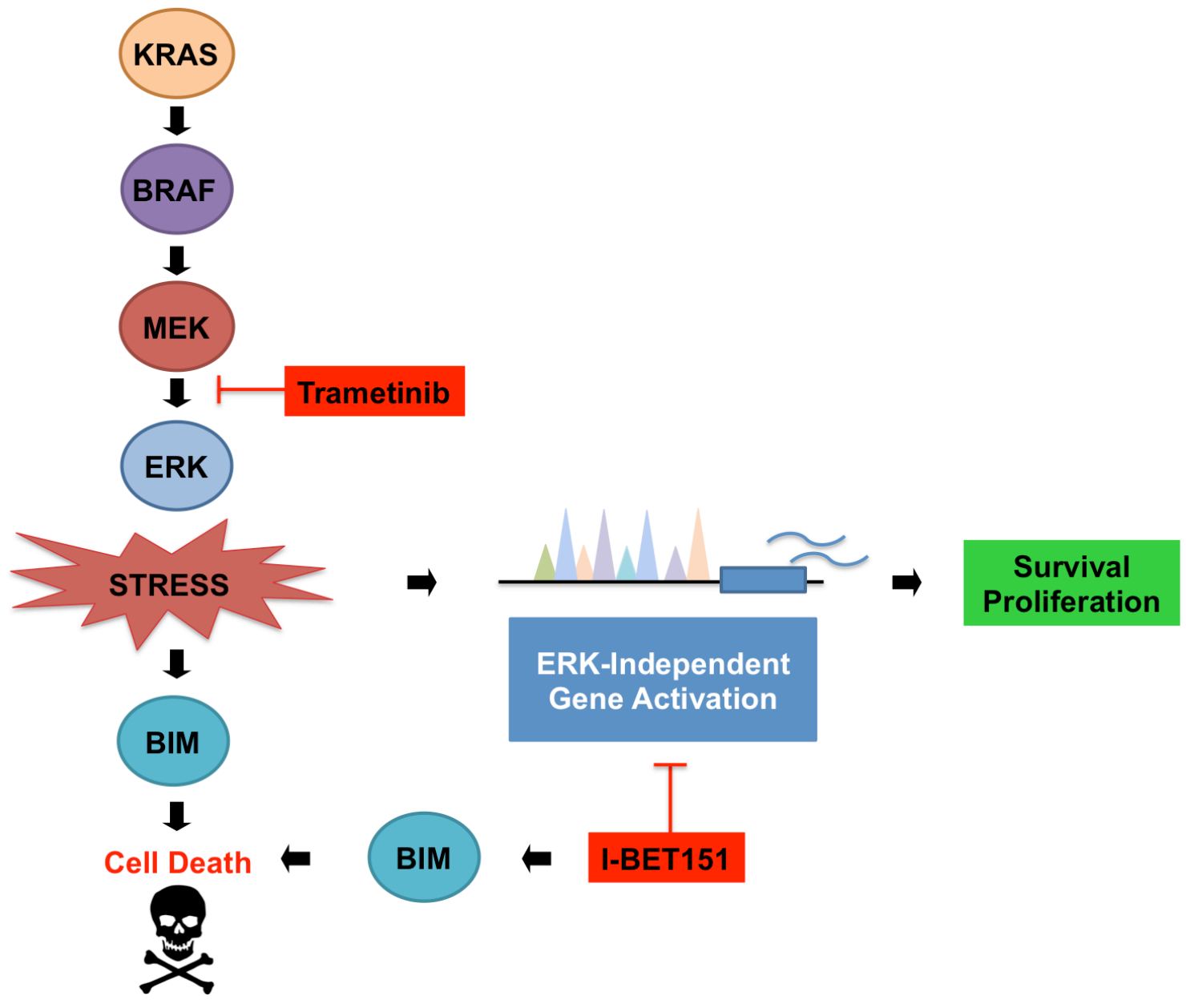


Figure 5. Trametinib and I-BET151 as a novel combinatiorial therapy in PDAC. PDAC cells harboring a constitutively activating mutation in KRAS are reliant on the classical MAPK signaling cascade for growth and survival. Inhibition of this cascade with trametinib incudes cellular stress and requires the cells to initiate ERK-independent signaling mechanisms. Activation of these alternative pathways requires faithful deposition of the transcriptional machinery at ERK-independent genes. By displacing BRD4, I-BET151 will disrupt localization of transcriptional machinery at regions of trametinib-induced gene activation. Cells will, therefore, fail to overcome the stress induced by blockade of the MAPK pathway and will undergo cell death. We hypothesize that combinatorial treatment with trametinib and I-BET151 will reduce the population of drug-refractory cells by preventing cellular reprogramming and inducing cell death. 


\section{RESULTS}

\section{Trametinib treatment of PDAC tumor spheres selects for a refractory population of cells}

The results of testing therapeutic efficacy differ between standard tissue culture models and in vivo xenograft studies. In this study, we utilize a threedimensional (3D) tumor sphere tissue culture model of patient-derived tumor cell lines in an effort to more faithfully recapitulate aspects of the tumor architecture and the tumor microenvironment (Fig. 6A). Both 366 and 608 PDAC cell lines were derived from metastatic tumors excised from the livers of patients. These cell lines harbor mutations commonly found in PDAC tumors, including mutations in the KRAS, TP53, and SMAD4 genes. In the interest of studying MEK inhibition in this model, we performed an immunoblot to assess activation of the MEK/ERK pathway in lysates from 2D and 3D cultures as well as from tumor. As shown in Figure 6B, the tumor samples and 3D cultures have increased levels of phosphorylated ERK and MEK compared to 2D cultures, indicating increased activation of the KRAS/MEK/ERK pathway (Fig. 6B). Additionally, 3D and tumor samples had lower levels of epithelial marker E-cadherin compared to 2D, and higher levels of mesenchymal marker Vimentin compared to 2D. These results illustrate that tumor spheres, compared to conventional monolayer cultures, more similarly model aspects of tumor biology.

Previous work in our lab has shown that NSCLC cells cultured in 3D can enrich for CICs (Kumar et al., 2013; Wamsley et al., 2015). Culturing PDAC cells in 3D also initiates upregulation of CIC-associated genes. As shown by qPCR, 
mRNA levels of stem-cell transcription factors Myc, SOX2, and KLF4 are upregulated in 366 3D cultures compared to 2D 366 cultures; SOX2 and KLF4 are similarly upregulated in 3D 608 cultures (Fig. 6C). Importantly, both cell lines upregulated expression of $A B C$ transporter genes $A B C C 1$ and $A B C G 2$ when taken through tumor sphere culture (Fig. 6D). Stem cell transcription factors and the $A B C$ transporters are associated with drug resistance and poor clinical outcome (Długosz and Janecka, 2016; Luo et al., 2013; Sugano et al., 2015). Testing trametinib in tumor spheres may elucidate genes important for resistance to MEK inhibition.

To establish an effective treatment concentration for trametinib in this tumor sphere model, we performed a dose curve and found that $100 \mathrm{nM}$ maximally reduced cell count following tumor sphere replating; further increasing the concentration to $1000 \mathrm{nM}$ did not further decrease cell count (Fig. 6E). Importantly, we observe that there is a trametinib-refractory population of cells within the 366 and 608 tumor spheres. We selected the $100 \mathrm{nM}$ treatment dose for the remainder of the experiments, unless otherwise stated.

The upregulation of $M y c$ has been proposed as a potential resistance mechanism to MEK inhibitors, however, we observe downregulation of Myc expression by qRT-PCR and a loss of Myc protein by immunoblot in tumor spheres treated with trametinib (Fig. 6F and 6G) (Sun et al., 2014). We hypothesize that KRAS mutant PDAC cells treated with trametinib need to undergo transcriptional reprogramming in order to overcome their dependence on survival signals potentiated by the KRAS/MEK/ERK pathway. Transcriptional 
reprogramming is dependent on the complex formation of transcriptional machinery at the enhancer regions of important genes. Transcriptional activation of genes requires the accumulation the Mediator complex, RNA Polymerase II (Pol II), and Positive transcription elongation factor b (P-TEFb) to enhancer elements and gene promoters. The BET proteins are central to recruitment of these core factors to the chromatin for effective transcription. Thus, we hypothesize that if we block the ability of BET proteins to localize to the chromatin, cells would fail to transcriptionally reprogram. Such an inhibition would be predicted to prevent PDAC cells from overcoming oncogene addition to the KRAS/MEK/ERK pathway. If our hypothesis were correct, this would cause cellular stress and effective induction of apoptosis in the presence of MEK and BET inhibitors. 


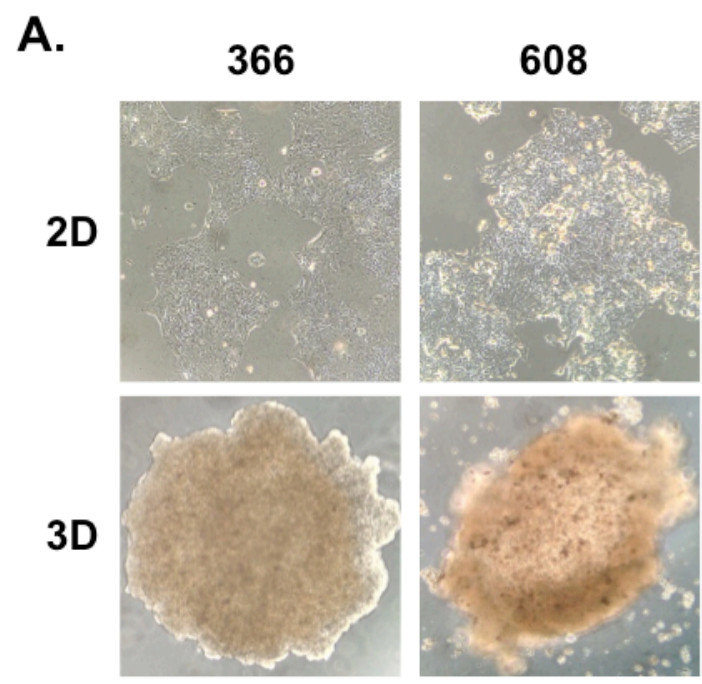

B.

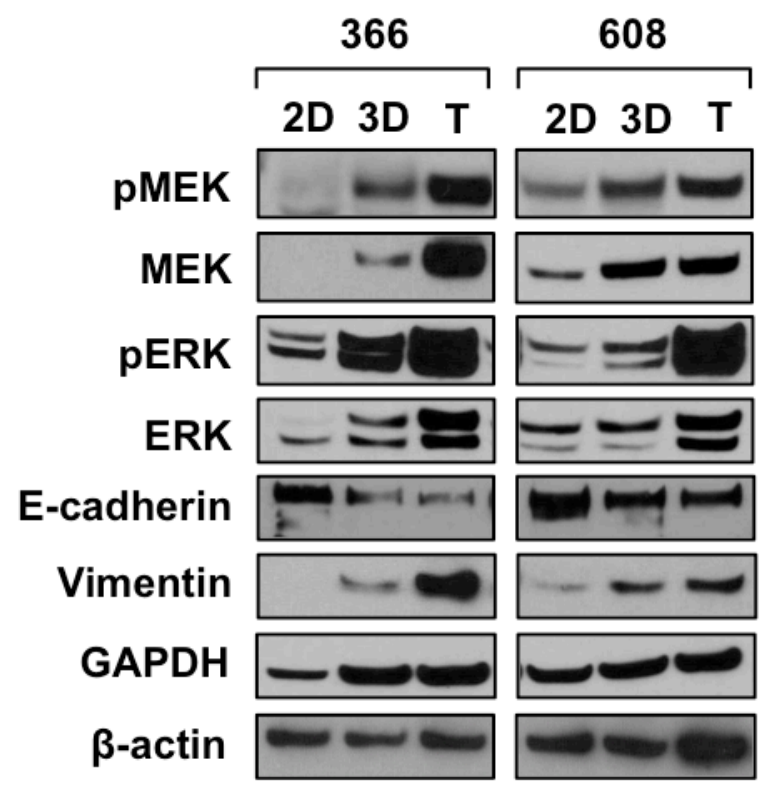


c.
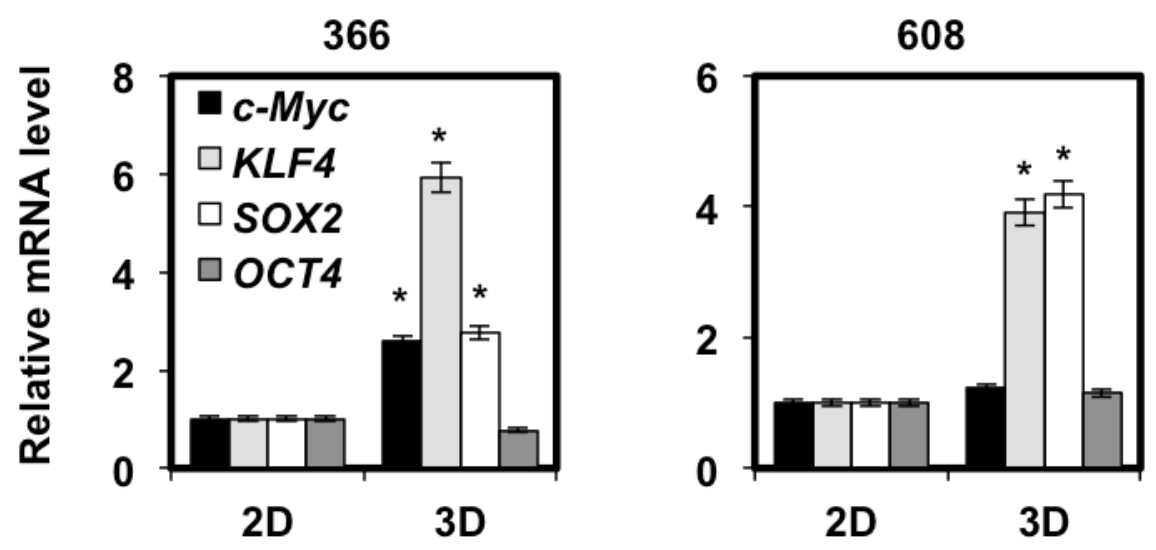

D.
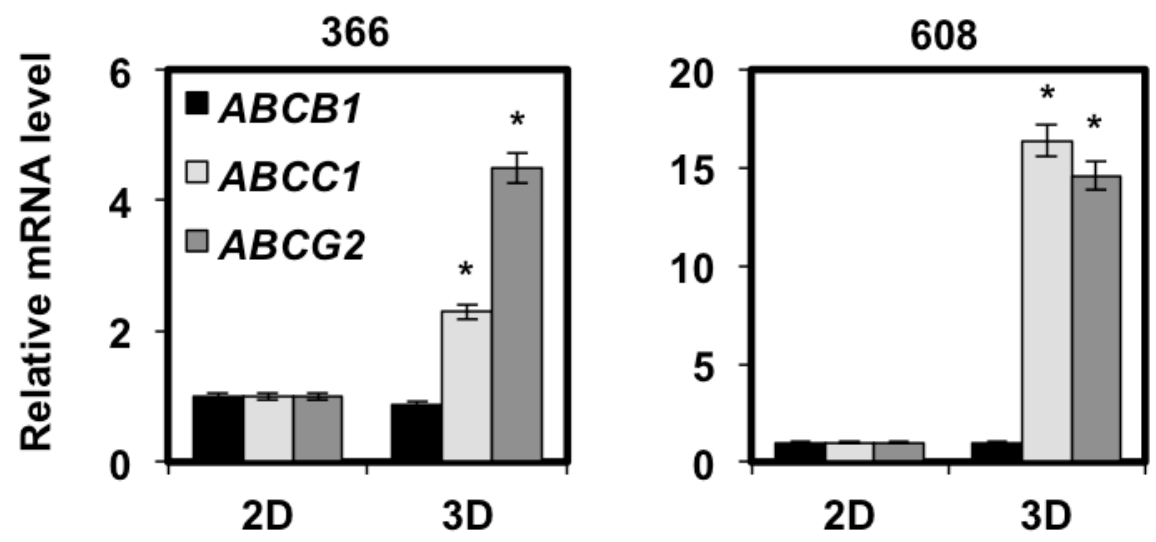
E.

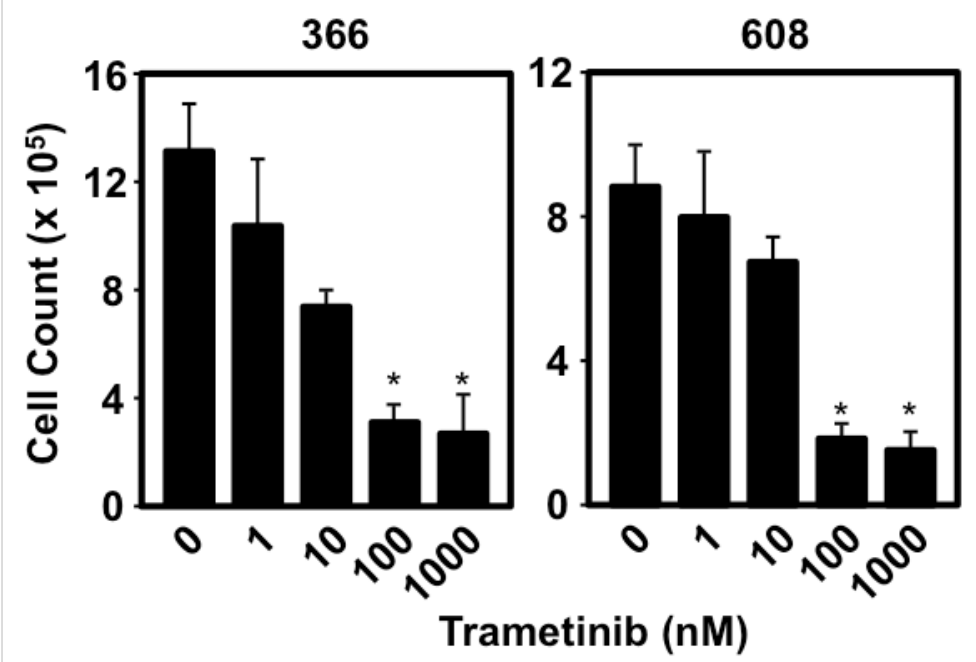

F.

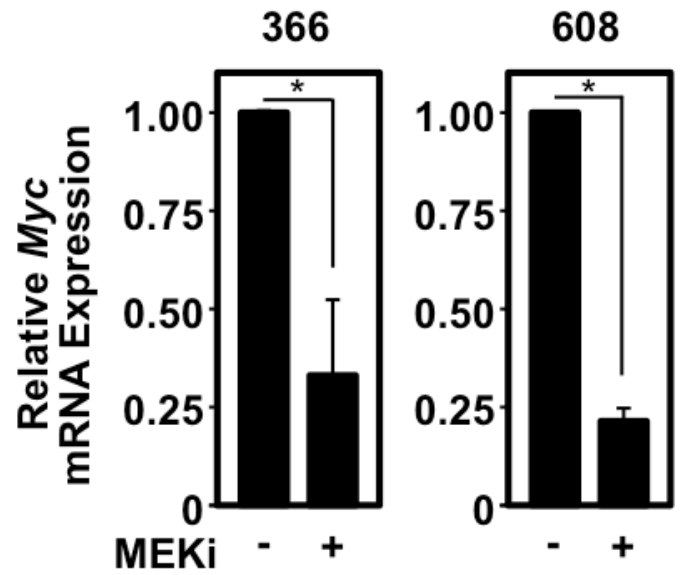

G.

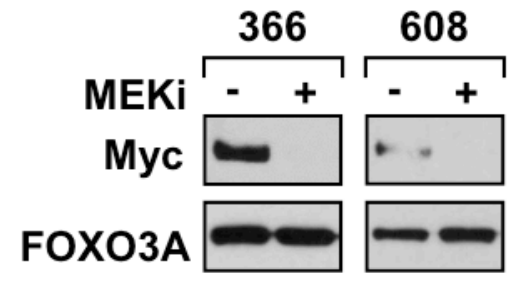


Figure 6. PDAC tumor spheres exhibit resistance to trametinib. (A) Light microscopy of 366 and 608 cultures in 2D and 3D cultures, 10X objective. (B) Immunoblot for protein levels in 2D, 3D and tumor lysates; blots were probed for markers of MEK/ERK activity and mesenchymal phenotypes. GAPDH serves as a loading control for 608 cultures, while $\beta$-actin serves as a loading control for 366 cultures. (C) qRT-PCR for stem cell transcription factors (Myc, KLF4, SOX2, and OCT4) in 2D and 3D cultures. (D) qRT-PCR for ABC transporters associated with multidrug resistance $(A B C B 1, A B C C 1$, and $A B C G 2)$ in $2 \mathrm{D}$ and 3D cultures. Data collected in qRT-PCR experiments were normalized to GAPDH. (E) Tumor spheres of 366 and 608 were either left alone or treated with increasing doses of trametinib for 96 hours before being replated on tissue culture dishes for 24 hours and counted. (F \& G) Treatment of 366 and 608 tumor spheres with trametinib (100 nM, 18 hours) results in the loss of Myc mRNA and protein expression, as detected by qRT-PCR (F) or immuoblot (G). 


\section{Combination of I-BET151 and trametinib synergize to reduce the population of refractory PDAC cells in tumor sphere culture}

We sought to test whether addition of I-BET151 could diminish the trametinib-refractory population of cells. To confirm inhibition of MEK and BET protein function with trametinib and I-BET151, we measured known functional outcomes of these drugs. MEK inhibition was tested by detecting phosphorylated ERK ( $p E R K$ ) levels using immunoblot; upon treatment with trametinib, we see reduced pERK levels indicating inhibition of MEK kinase activity (Fig. 7A) (Lindberg et al., 2014). I-BET151 function was assessed using qRT-PCR to measure transcriptional expression of FOSL1, a known BRD4regulated gene target (Lockwood et al., 2012). Figure 7B shows a decrease in FOSL1 mRNA expression upon treatment of tumor spheres with I-BET151.

To quantify the effects of the drug combination on cell number, tumor spheres were treated with trametinib and/or I-BET151 inhibitors twice over a 96hour period before being dissociated and replated onto tissue culture dishes. The clumps of cells were allowed to attach to the adherent plates overnight before being further dissociated in order to be accurately counted.

Representative phase contrast microscopy images of replated tumor spheres are shown in Figure $7 \mathrm{C}$. The combination treatment significantly reduced cell count compared to trametinib treatment alone in both 366 and 608 cultures (Fig. 7D). Crystal violet staining of fixed cells following treatment of tumor spheres for 96 hours indicates similar results (Fig. 7E \& F). 


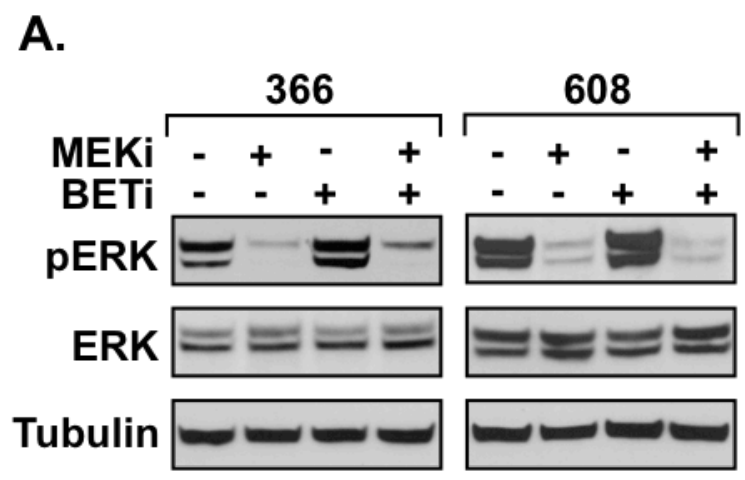

B.

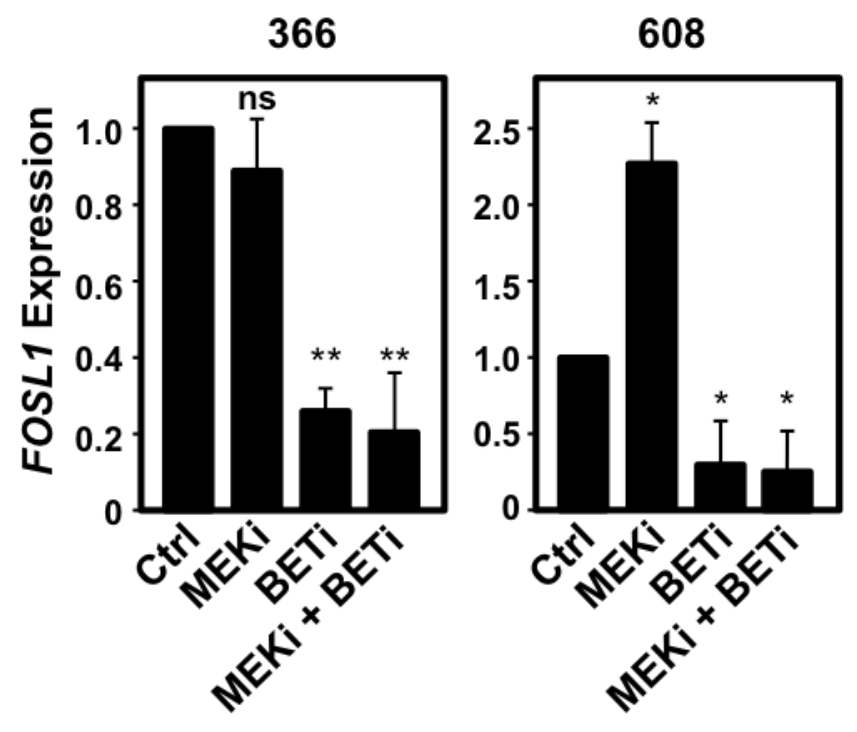




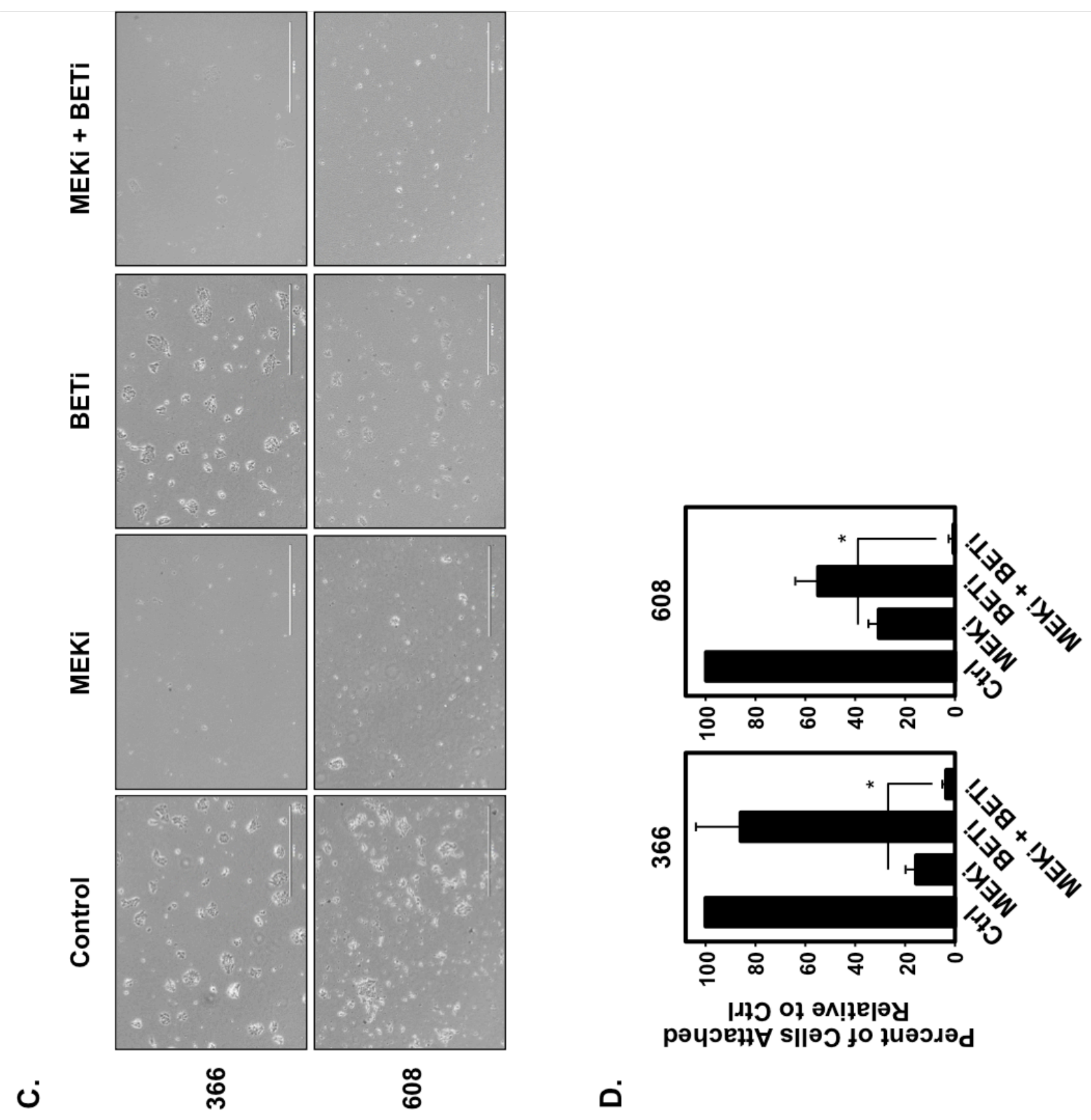


E.

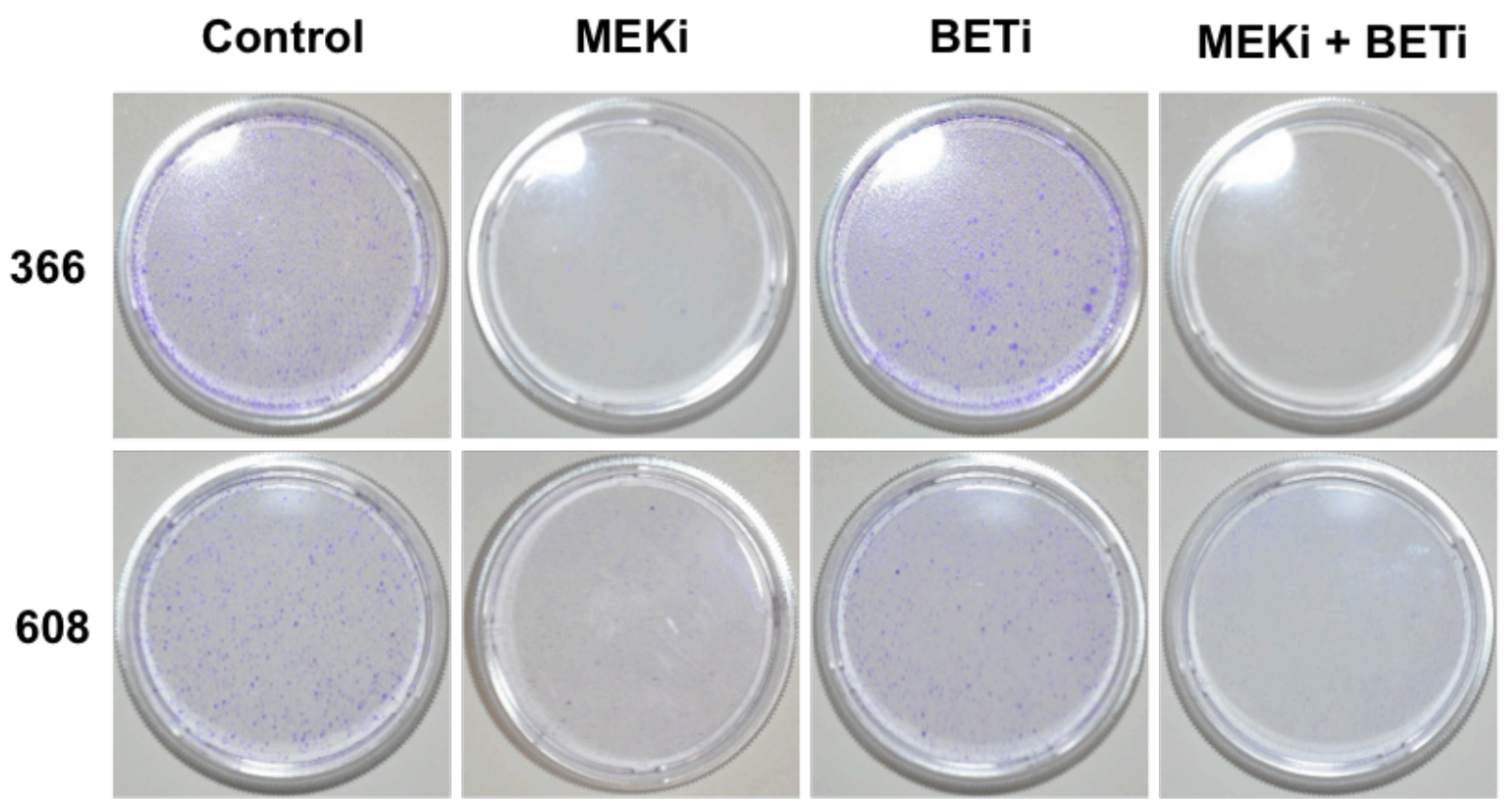

F.
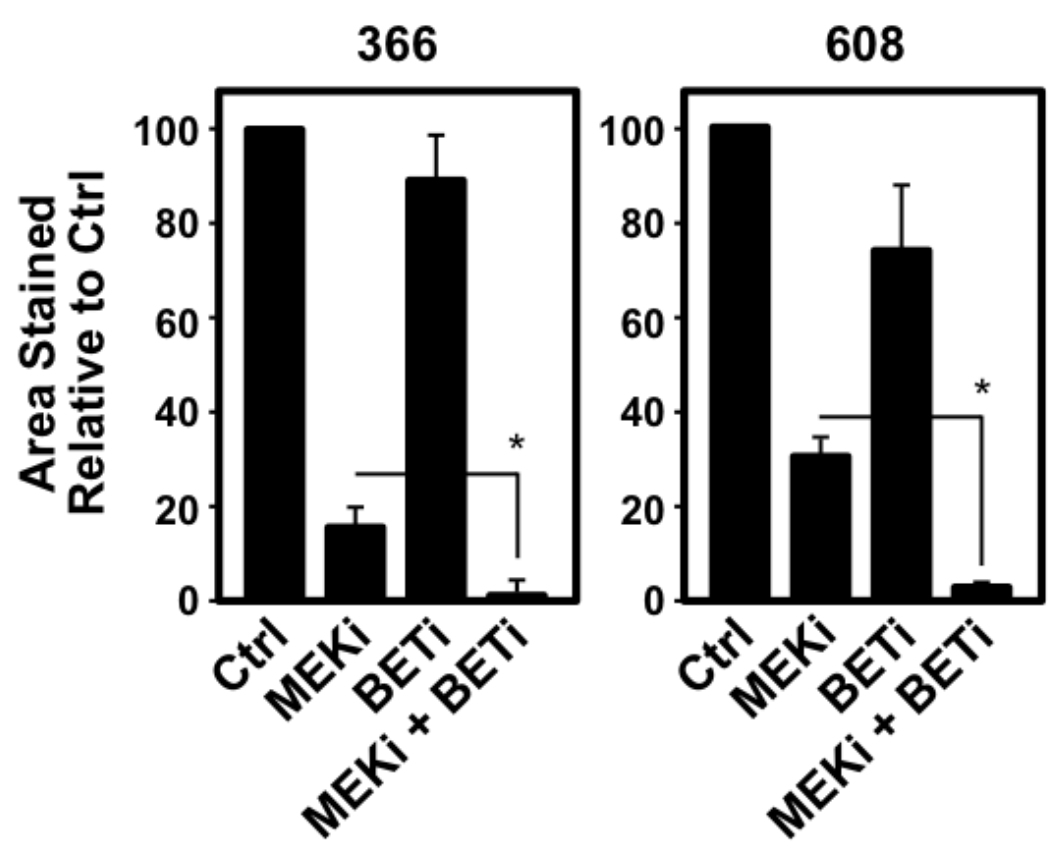


\section{Figure 7. I-BET151 treatment increases sensitivity of PDAC cells to}

trametinib. (A \& B) trametinib (100 nM) and I-BET151 (10 $\mu \mathrm{M})$ inhibitors are effective in tumor spheres by their ability to suppress immunoblot detection of phospho-ERK activity (A), and qRT-PCR analysis of FOSL1 transcription (B), respectively. Results were normalized to GAPDH. (C) Representative images of replated tumor spheres that had been treated with indicated inhibitors for 96 hours before dissociation and replating onto tissue culture dishes (phase contrast microscopy, 10X magnification). (D) Cell counting data of three independent experiments described in (C), plotted relative to control. (E) Crystal violet staining of fixed cells that had been treated in tumor spheres with indicated inhibitors for $96 \mathrm{hr}$ before dissociation and replating onto tissue culture dishes. (F) Quantitation of the area of each plate stained with crystal violet in $(E)$; each condition is plotted relative to area stained in the control. 


\section{Addition of I-BET151 increases trametinib-induced apoptosis in tumor spheres}

Due to the significant reduction in cell numbers, we hypothesized that trametinib and I-BET151 plus trametinib treated cells were undergoing apoptosis in the 3D cultures. During apoptosis, effector caspase enzymes cleave substrates such as nuclear lamins, DNase inhibitors, DNA repair enzymes, and cytoskeletal proteins - this results in chromatin condensation and DNA fragmentation. The nuclear content of cells can be measured by FACS analysis of DAPI stained nuclei; cells with nuclear content less than that of cells in the G1 (SubG1) phase of the cell cycle (2n) have undergone apoptosis. FACS analysis of DAPI stained cells show 366 and 608 PDAC cells exhibit a baseline level of SubG1 $(<2 n)$ cells in 3D culture, which was significantly increased by incubation of the tumor spheres with trametinib (Fig. 8A). In both cell lines, dual treatment with the MEK and BET inhibitors significantly increases the SubG1 fraction of cells compared to trametinib alone.

To confirm activation and commitment to apoptotic signaling, we measured the abundance and activity of effector enzyme caspase-3. Following 24 hours of drug treatment, protein levels of the active cleaved form of caspase-3 were measured by immunoblot; we also probed for a substrate of caspase-3, PARP. The highest levels of cleaved caspase- 3 and cleaved PARP were observed in the combinatorial treatment for both 366 and 608 cell cultures (Fig. 8B). Additionally, tumor spheres treated with inhibitors for 48 hours were collected for cell lysis, normalized for protein content and assayed for caspase-3 
activity. Although the MEK inhibitor was able to significantly induce caspase-3 activity, the dual combination further increased caspase-3 activity compared to trametinib alone (Fig. $8 \mathrm{C}$ ). These data indicate that the combination of trametinib and I-BET151 is more effective in inducing intrinsic apoptosis than trametinib treatment alone. 

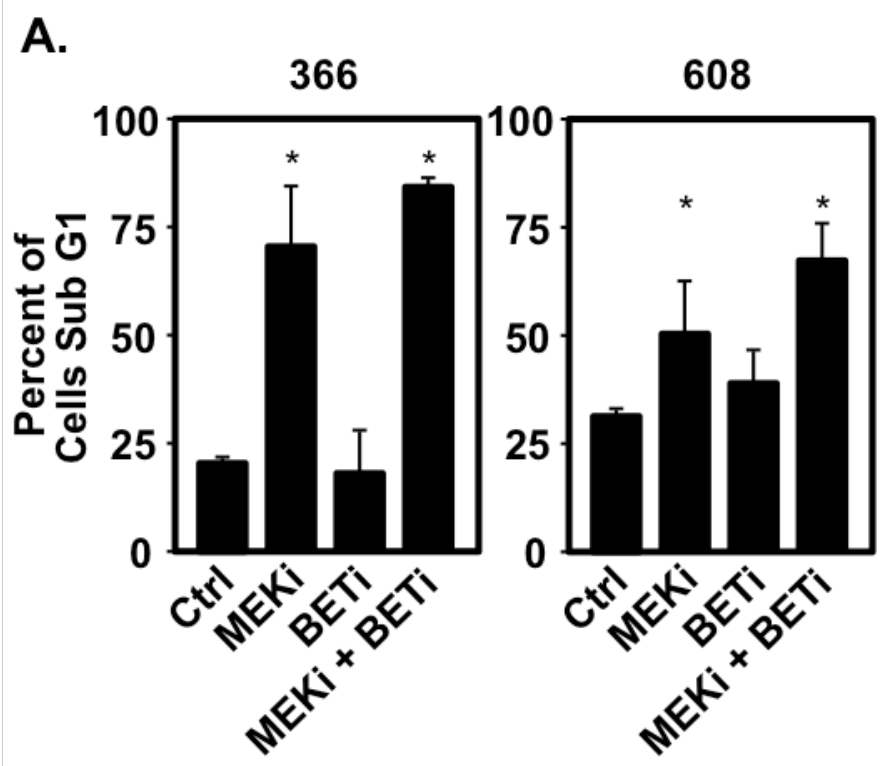

B.
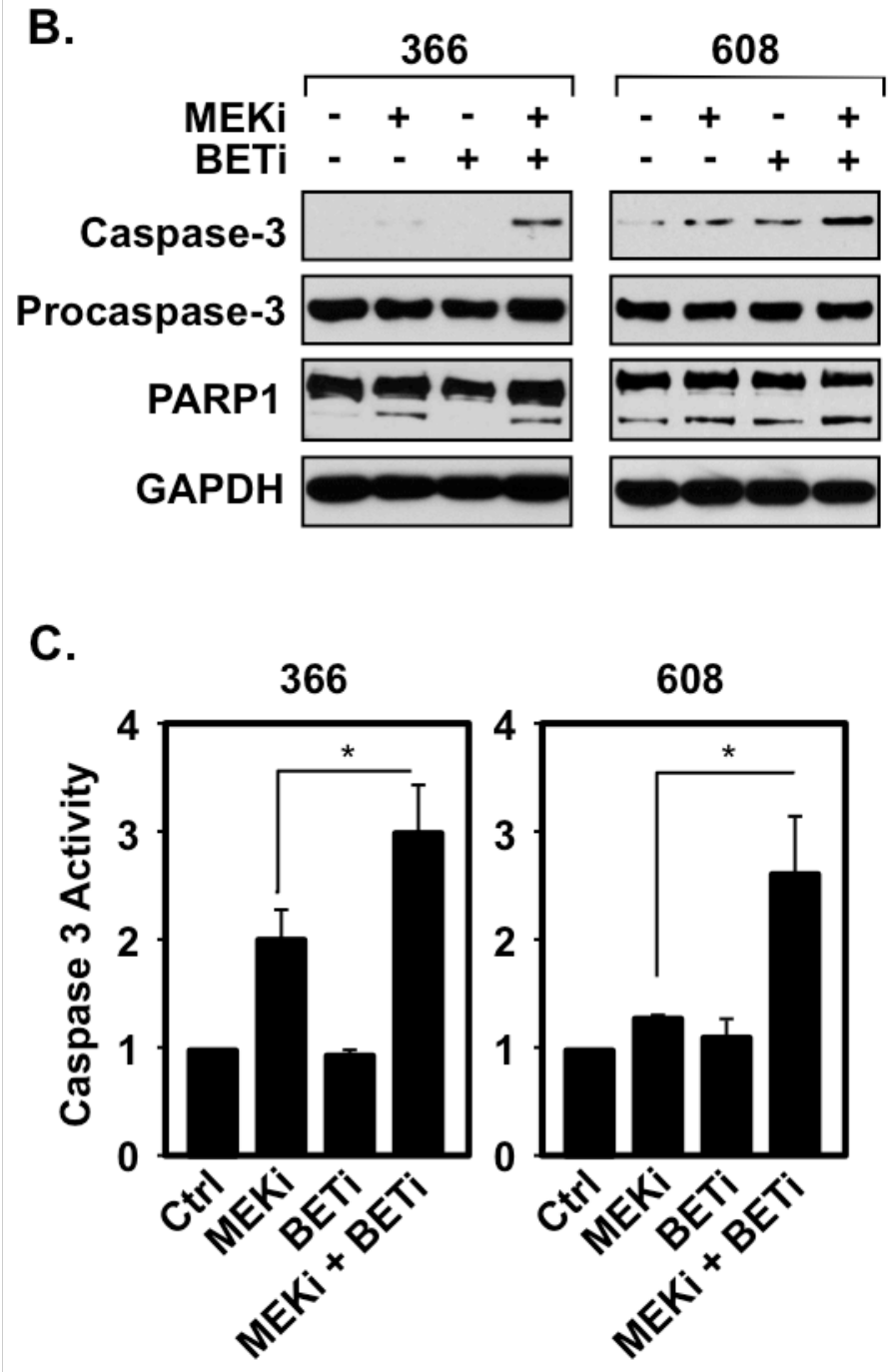
Figure 8. Combinatorial treatment with trametinib and I-BET151 induces apoptosis in PDAC tumor spheres. (A) FACS analysis for sub G1 $(<2 n)$ nuclear content of tumor spheres incubated with the indicated treatments for 96 hours (366) or 72 hours (608). (B) Immunoblot showing increased protein levels of activated executioner enzyme caspase 3 and one of its substrates PARP. GAPDH served as a protein loading control. (C) Caspase 3 activity measured in cell lysates collected from tumor spheres treated as indicated for 48 hours. 


\section{Individual and combinatorial treatments using trametinib and I-BET151} increase BIM levels.

BIM is known as the activator of apoptosis. Under conditions of cellular stress, BIM translocates from the microtubules to the mitochondria to activate the cell death program. Previous studies have shown that ERK can phosphorylate and stimulate BIM for ubiquitin-mediated degradation (Harada et al., 2004; Wang et al., 2011). By preventing ERK activation, we predicted to see a decrease in phosphorylated BIM levels and an increase in overall BIM levels. Additionally, inhibitors of BET proteins have been shown to increase BIM transcription (Gallagher et al., 2014b; Patel et al., 2014). We hypothesize that the increase in apoptosis following treatment with these inhibitors is due to an increase in BIM levels within the cells. By immunoblot, we observed increased levels of BIM protein with trametinib alone and I-BET151 alone, however the highest levels of BIM are induced by the combination treatment (Fig. 9A). Treatment with IBET151 alone increased the pool of phosphorylated BIM. Detection of phosphorylated BIM was significantly reduced in cells treated with trametinib, as excepted (Fig. 9A).

Transcription of BIM was induced in samples treated with I-BET151, but not in the trametinib alone sample (Fig. 9B). These data suggest that trametinib post-transcriptionally regulates an increase of BIM protein, while I-BET151 increases cellular BIM levels transcriptionally. Of interest, I-BET151 upregulates production of the $B I M_{L}$ and $B I M_{S}$ splice forms of $B I M$, but not $B I M_{E L}$. The mechanism by which this drug induces transcription of BIM and its splice forms 
has been probed in other studies, but remains unknown (Gallagher et al., 2014b; Li et al., 2016).

Though I-BET151 was effective at inducing expression of BIM as a single-agent, treatment with I-BET151 alone was not effective at inducing caspase-3 activity or cell death (as shown in Fig. $8 \mathrm{C}$ and $8 \mathrm{~A}$, respectively). This suggested that upstream events in the apoptotic signaling pathway were being disrupted. To test whether BIM was able to translocate to the mitochondria to activate apoptosis, we performed cell fractionation followed by immunoblot. We found that, despite high levels of BIM protein, cells treated with I-BET151 had lower levels of BIM at the mitochondria compared to cells treated with trametinib alone or in combination with I-BET151 (Fig. 9C).

Additionally, cells treated with trametinib had higher levels of BAX and BAK at the mitochondria; these proteins are responsible for forming a pore in the mitochondrial membrane responsible for the release of cytochrome $\mathrm{C}$ that activates the caspase cascade and, eventually, cellular apoptosis. It is unclear what is preventing BIM from translocating to or associating with the mitochondria, but this disassociation explains why I-BET151 treatment alone does not result in high levels of cell death despite induction of BIM transcription. The combinatorial effects of the two inhibitors, however, result in the accumulation of apoptotic proteins at the mitochondria. This correlates with the increased caspase- 3 activity and cell death data previously described in Figure 8. These studies support the use of this combinatorial therapy to stimulate apoptosis in PDAC cells. 
A.
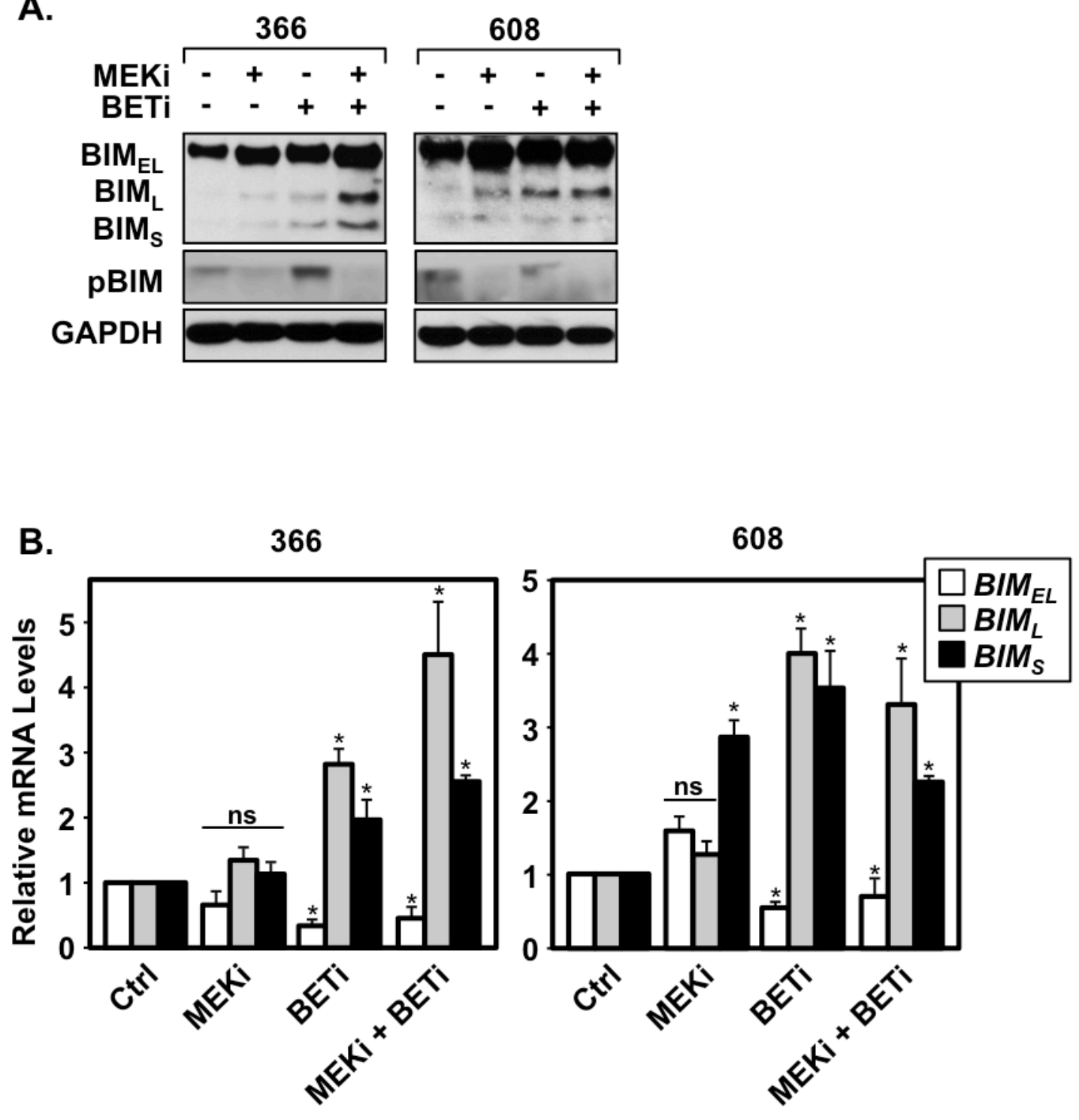
c.

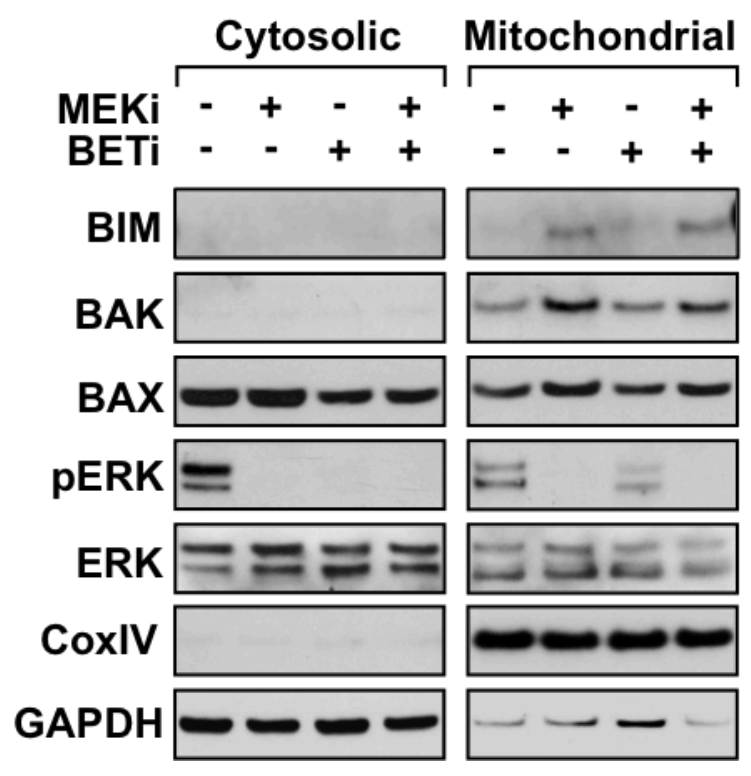


Figure 9. Individual and combinatorial treatments of trametinib and/or IBET151 increase mitochondrial levels of BIM. (A) Immunoblot showing trametinib and I-BET151 treatment increases BIM protein levels both individually and in combination. (B) I-BET151 treatment stimulates transcription of $B I M_{L}$ and $B I M_{S}$ isoforms in both 366 and 608 tumor spheres as detected by qRT-PCR. Results were normalized to GAPDH. (C) Immunoblot of cytosolic and mitochondrial fractions isolated from trametinib and I-BET151 treated tumor spheres. Trametinib treated samples display elevated mitochondrial protein levels of proapoptotic proteins BIM, BAK and BAX. CoxIV and GAPDH served as protein loading controls for the mitochondrial and cytosolic fractions, respectively. 


\section{Combinatorial treatment reduces markers of EMT and CIC in PDAC cells.}

As shown in Figure 6, there is a population of drug-refractory cells in 366 and 608 tumor spheres treated with a high dose of trametinib. Either the cells do not rely on the KRAS/MEK/ERK signaling pathway to survive, or the cells are adapting to the drug conditions by shifting their signaling mechanisms to activate alternative survival pathways. Previous studies have shown that refractory populations emerge in tumors treated with targeted inhibitors or conventional chemotherapy. Moreover, these resistant populations are enriched for markers of EMT and stemness (Dorris et al., 2016; Du et al., 2011; Sprenger et al., 2013). We wanted to know if acute treatment of tumor spheres with trametinib enriches EMT and CIC markers. At the same time, we were interested in the possibility of reducing these phenotypes with I-BET151.

qRT-PCR analysis of 3D 366 shows trametinib treated tumor spheres induce EMT masterswitch transcription factors TWIST1 and SNAI2 (Fig. 10A). Interestingly, the addition of I-BET151 diminishes the induction of TWIST1 and SNAI2 (Fig. 10A). ZEB2 was inhibited by all drug conditions in 366 . In the 608 cells, TWIST1, SNAI1 and ZEB2 were upregulated following trametinib treatment; all three genes remained at control levels with the addition of IBET151 to trametinib. Also, the combination treatment reduced expression of SNAI2 in 608 cells (Fig. 10A). Culturing 366 and 608 cells in 3D culture was sufficient to induce a more mesenchymal phenotype compared to $2 \mathrm{D}$ cultures (Fig. 6B). Treatment with the combination of MEK and BET inhibitors reduced mesenchymal markers Fibronectin and Vimentin in both 366 (48 hour treatment) 
and 608 (96 hour treatment) tumor spheres (Fig. 10B). Generally, E-cadherin is lost during epithelial to mesenchymal transition (EMT) and N-cadherin is induced. Interestingly, E-cadherin was not affected by the inhibitor combination in 366 cultures and was reduced in 608 cultures (Fig. 10B). Unlike E-cadherin, Ncadherin was not expressed in 366 or 608 cultures as detected by immunoblotting (data not shown).

Evidence supports the theory that EMT promotes cellular invasive and migratory phenotypes, characteristics necessary for metastasis. To assess the effects of trametinib, I-BET151 and the combination on migration and invasion, we attempted to perform transwell assays using spheroid-cultured cells. Due to technical difficulties, we were unsuccessful at carrying out these assays (data not shown). As an alternative, we performed scratch assays to measure the effects of the inhibitors on migration in 2D cultures. Cells were plated to confluency before being scratched, treated with inhibitors $(50 \mathrm{nM}$ of trametinib, $10 \mu \mathrm{M} \mathrm{I-}$ BET151), and imaged over time. While trametinib alone significantly reduced migration, the combination was even more effective at inhibiting migration over the time frame analyzed (Fig. $10 \mathrm{C} \& \mathrm{D}$ ). These results indicate that the addition of I-BET151 along with trametinib reduced the migratory capacity of PDAC cells. Interestingly, trametinib increased expression of cancer stem cell transcription factors SOX2, OCT4, and KLF4 in 3D 366 cultures (Fig. 11A). Single-agent treatment with I-BET151 is able to significantly decrease expression of SOX2. When used in combination, I-BET151 mitigates the trametinibmediated induction of SOX2, OCT4, and KLF4 (Fig. 11A). In 608, the only CIC 
transcription factor upregulated following acute treatment with trametinib was SOX2; this was significantly reduced by the addition of I-BET151. Trametinib plus I-BET151 repressed expression of Nanog and KLF4 in 608 cultures. Finally, expression of Myc was strongly repressed by all drug conditions in both 608 and 366 cultures, indicating that each of the inhibitors were functioning in the cells.

Inhibition of $A B C$ transporters has been shown to overcome drug resistance of $\mathrm{CIC}$ populations (Sugano et al., 2015). Trametinib induced expression of $A B C B 1$ in 366 cultures and I-BET151 did not suppress this induction (Fig. 11B). In 608 cells, BET inhibition reduced baseline expression of $A B C B 1$. Importantly, we also saw that trametinib and I-BET151 were both able to reduce expression of $A B C C 1$ and $A B C G 2$ in 366 and 608 cells, with the lowest levels of mRNA detected in the combination treated samples (Fig. 11B). These data imply that trametinib and I-BET151 may be able to prevent or overcome the drug refractory nature of PDAC by altering the expression of $A B C$ transporters.

Next, we sought to characterize the stem-like population in the tumor spheres, as well as in response to drug treatment. FACS analysis for PDAC stem-cell surface markers EpCAM, CD44 and CD24 was performed, and cells were gated for triple positive staining of these proteins. The triple positive stemlike cell population significantly increased when 366 cells were cultured in 3D (20.4\%) compared to 2D (11.9\%) (Fig. 11C). The initial population of EpCAM+/CD24+/CD44+ cells in 2D 608 cultures was already high (22.4\%), and culturing the cells in 3D did not significantly change the population of CICs. Acute MEK inhibition did not alter the population of stem-like cells in 366 , 
however, treatment with the BET inhibitor significantly reduces the population of triple positive cells either alone or in combination with trametinib (Fig. 11C). In 608 cells, all treatments reduced the CIC population within the tumor spheres. Together, these data support the idea that the addition of I-BET151 in combination with trametinib can downregulate the expression of $\mathrm{CIC}$ transcription factors, as well as reduce the population of EpCAM+/CD24+/CD44+ cells in 3D 366 cultures. We postulate that this therapeutic approach has significant implications for reducing the population of drug-resistant cells and cancerinitiating cells within a tumor. 
A.

366

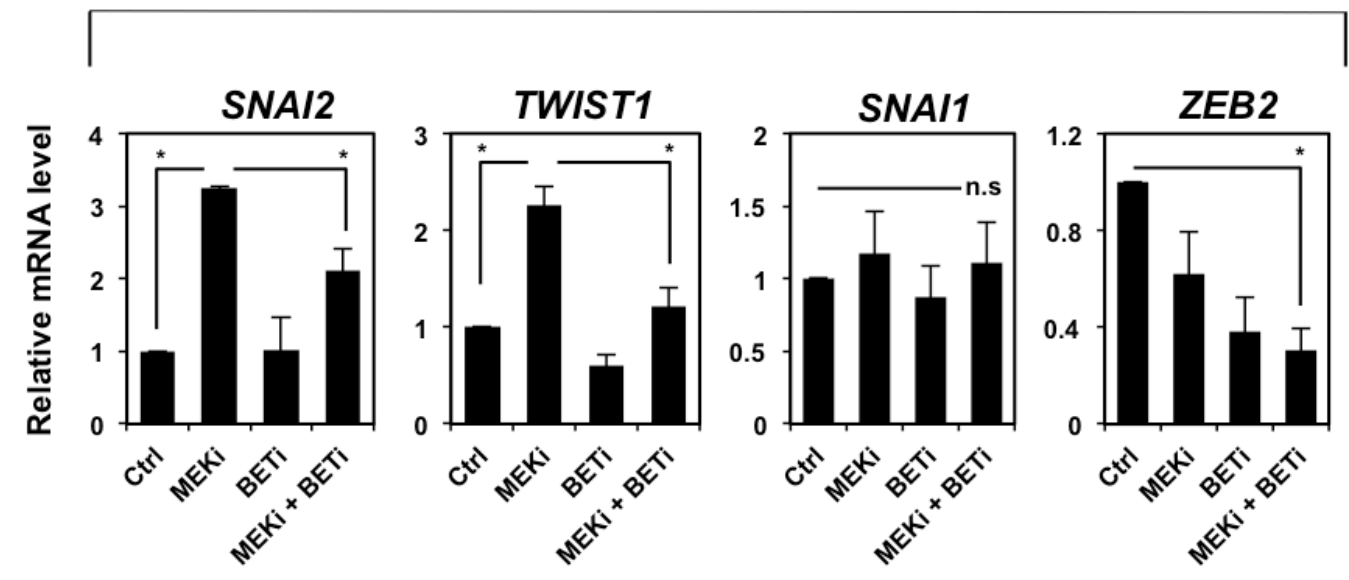

608

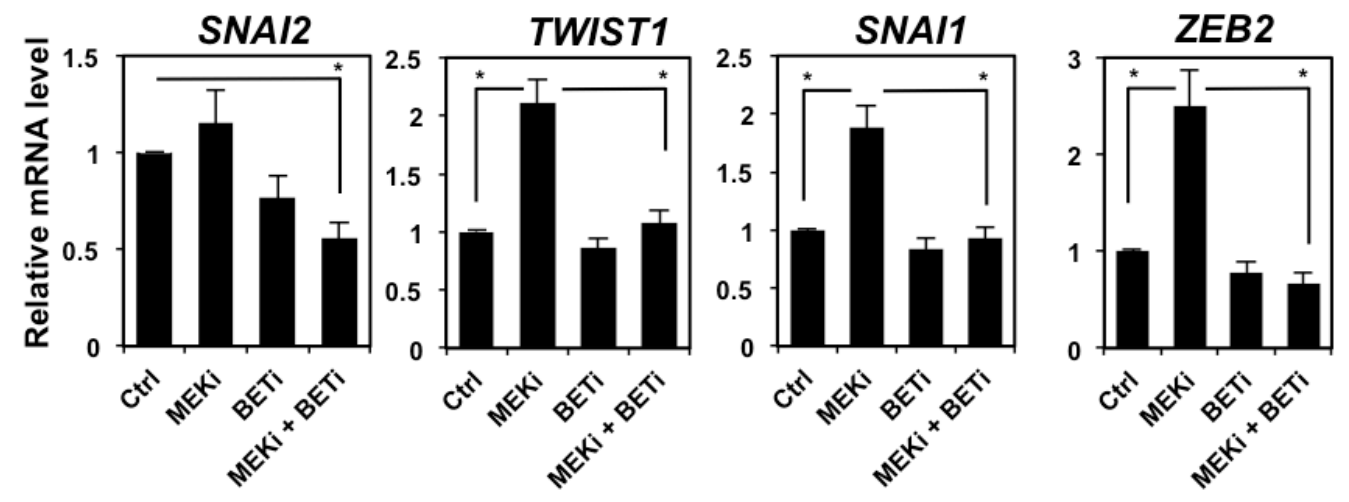

B.

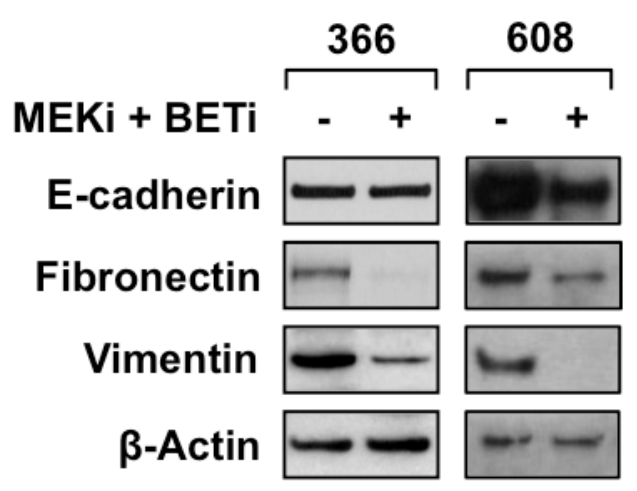




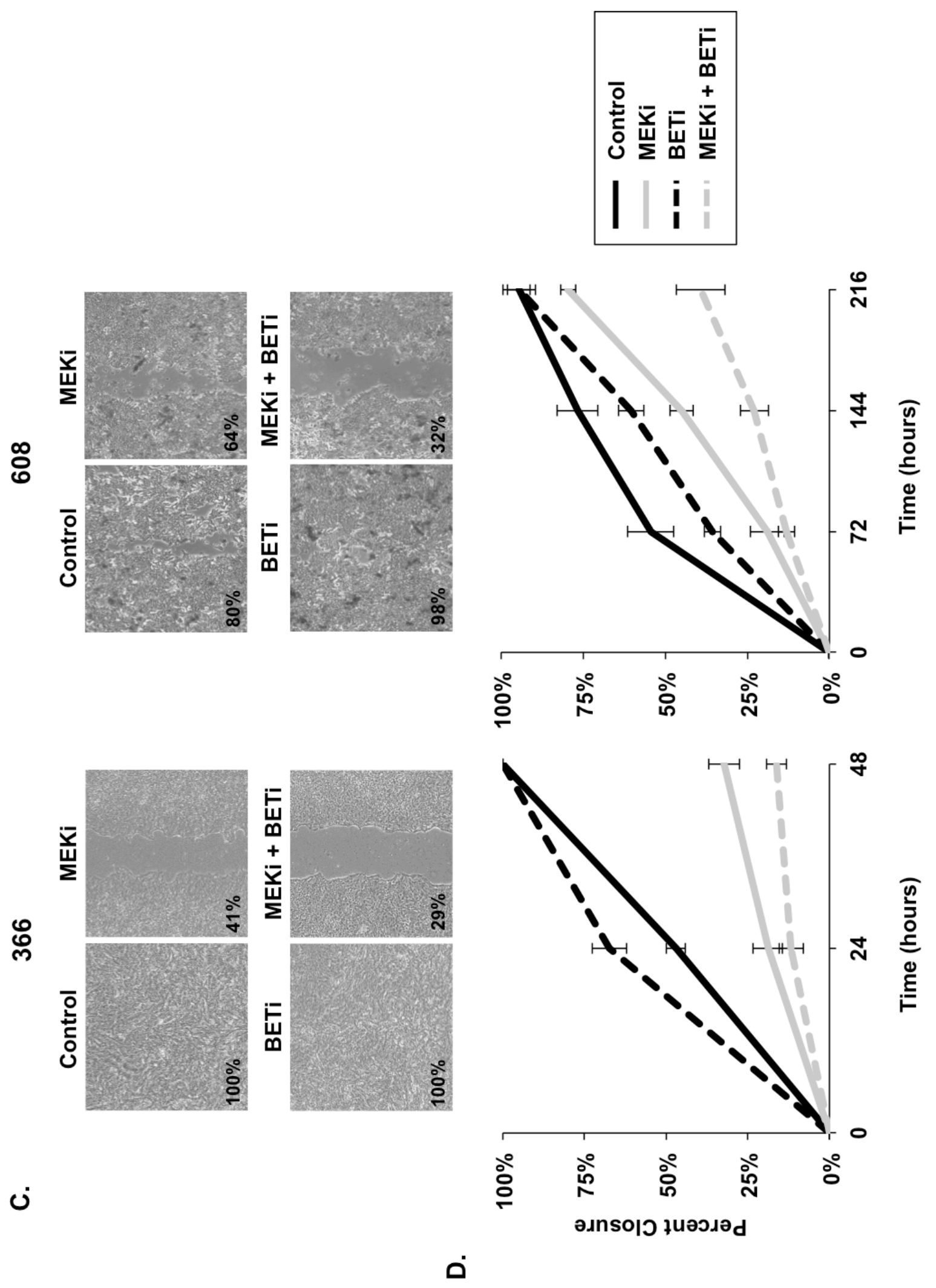


Figure 10. Combinatorial treatment reduces mesenchymal markers and migration phenotype in PDAC cells. (A) qRT-PCR for EMT masterswitch transcription factors (SNAI2, TWIST1, SNAI1, and ZEB2) in 366 and 608 tumor spheres treated with trametinib and I-BET151 for 18 hours; normalized to GAPDH. (B) Immunoblot for EMT markers in tumor spheres treated with trametinib + I-BET151 for 48 hours (366 cultures) or 96 hours (608 cultures). (C) Representative light microscopy images of scratch assays performed in 2D cell cultures during treatment with trametinib (50 nM) and I-BET151 (10 $\mu \mathrm{M})$. Images were taken 48 hours ( 366 cultures) and 216 hours (608 cultures) after the scratches were established. The percent of closure from the original scratch area is indicated in the lower left corner of each image. (D) Quantitation of the scratch assay over the indicated time points. The percent closure was equal to percent increase in the area of confluency relative to the area of the original scratch. 


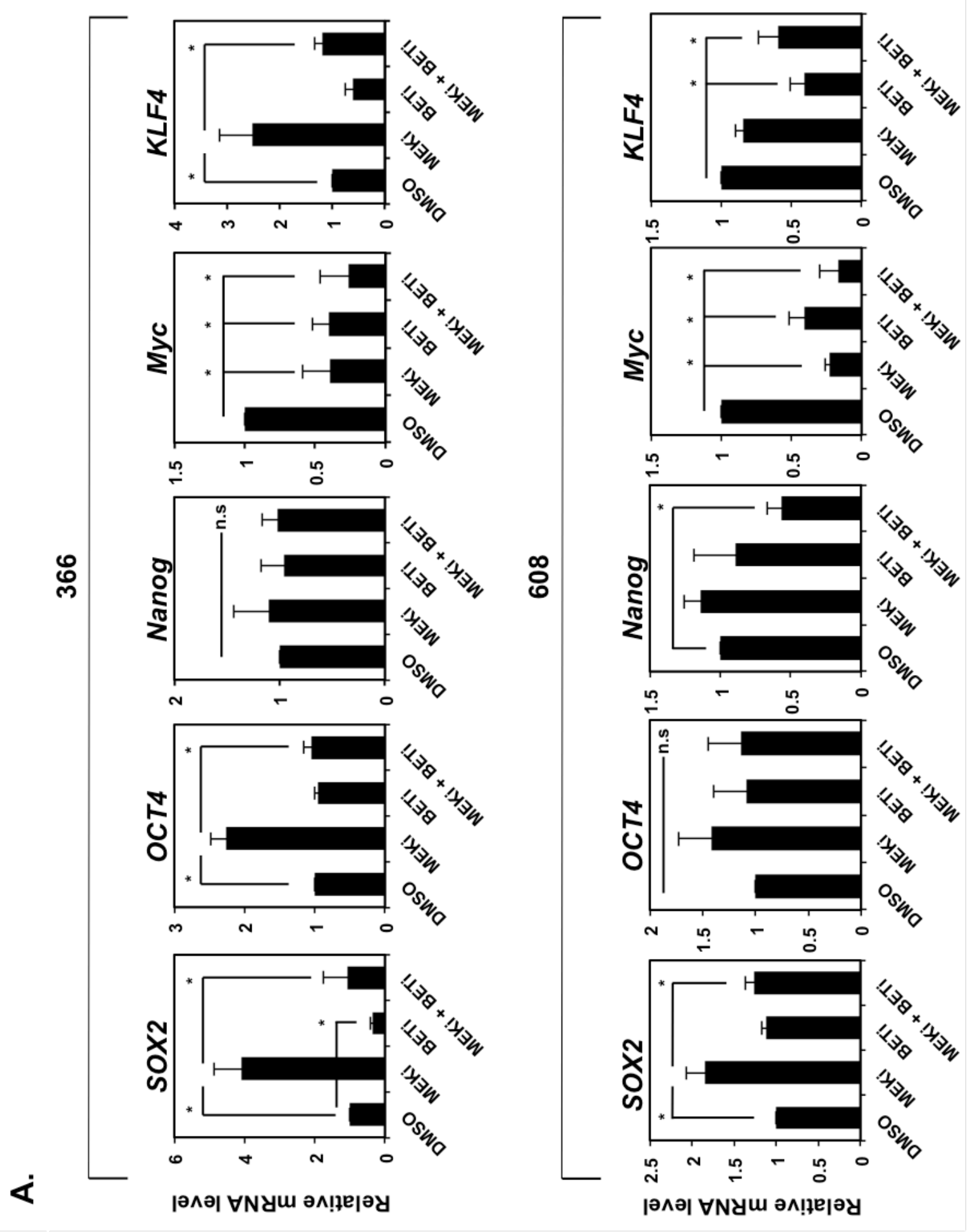


B.

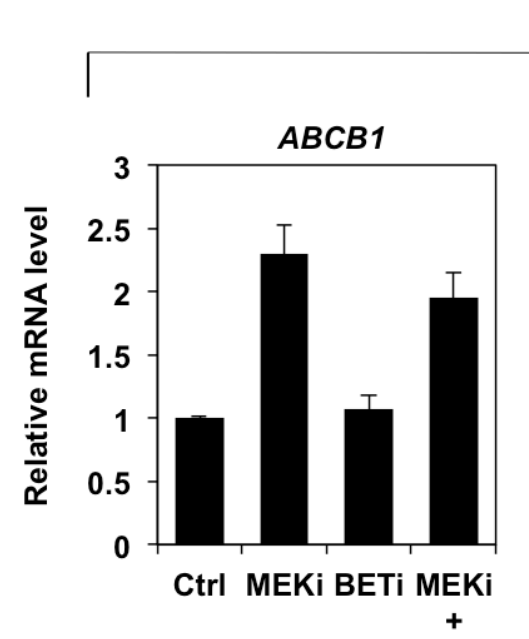

$\stackrel{+}{\text { BETi }}$
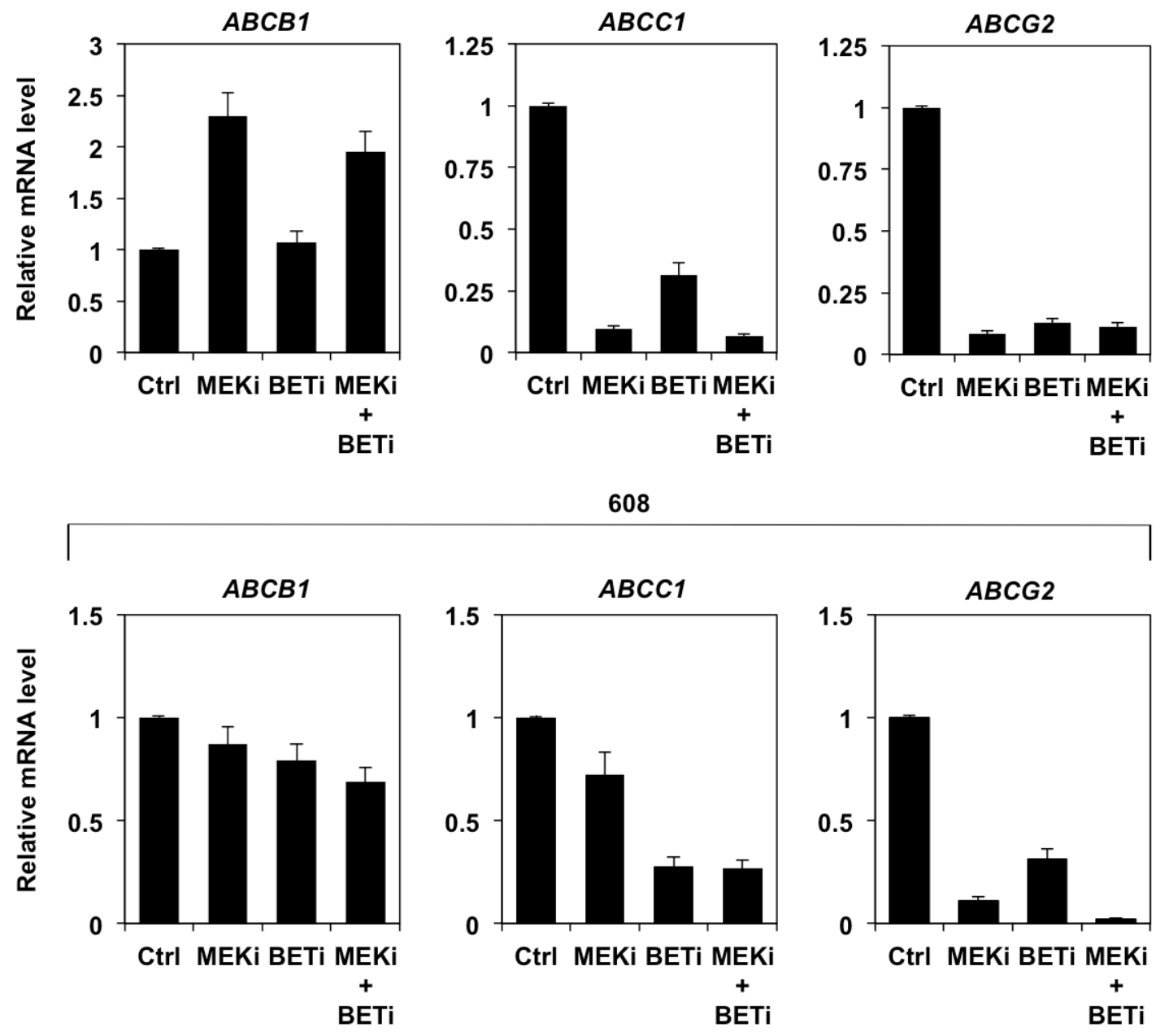
c.
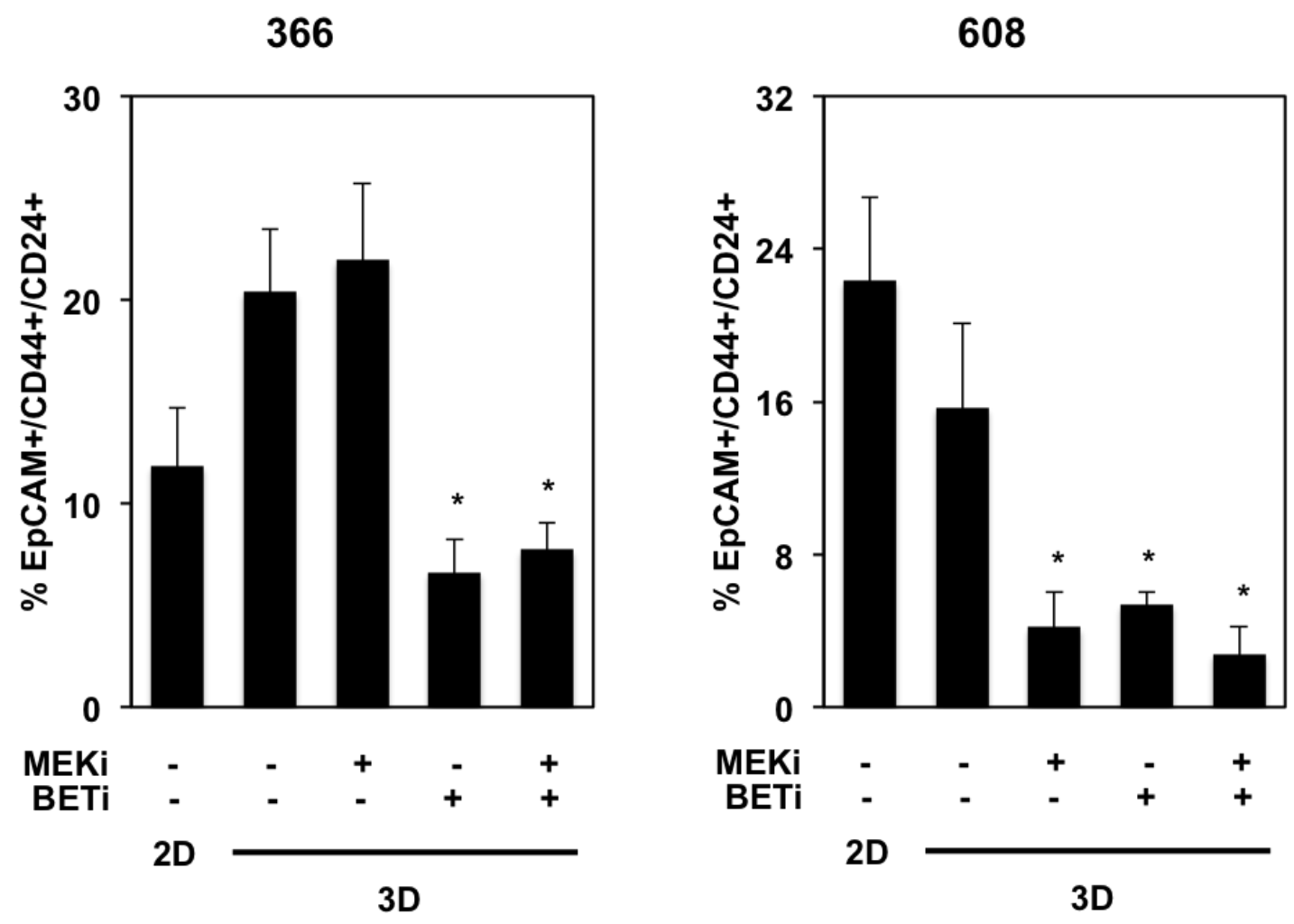
Figure 11. Combinatorial treatment reduces pluripotency markers in tumor sphere PDAC cultures. (A) Quantitation of mRNA levels for pluripotent transcription factors (SOX2, OCT4, Nanog, Myc, and KLF4) as measured by qRT-PCR in 366 and 608 tumor spheres treated with trametinib and I-BET151; normalized to $G A P D H$. (B) Quantitation of gene expression for $A B C$ drug transporter genes associated with drug resistance $(A B C B 1, A B C C 1$, and ABCG2) in 366 and 608 tumor spheres treated as indicated; qRT-PCR results normalized to GAPDH (C) Bar graph representative of FACS analysis quantifying the percentage of the cell population expressing pluripotent cell surface markers EpCAM+/CD44+/CD24+ in 366 and 608 cultures. 


\section{Treatment of orthotopic PDAC tumors with trametinib and I-BET151 induces tumor necrosis and fibrosis.}

Tumor explants from 366 were propagated in vivo were sutured onto the pancreata of athymic nude mice and allowed to grow for four weeks before being treated with trametinib, I-BET151 or a combination of the two drugs. Tumor volume, as measured by MRI, was used to establish a base-line tumor size before initiating targeted therapy. Consistent with previous studies, trametinibtreated tumors showed slower rates of growth compared to control tumors (Fig. 12A) (Lindberg et al., 2014; Walters et al., 2013). Although not as effective as trametinib, I-BET151 has the ability to significantly slow tumor growth in a PDX model of PDAC (Fig. 12A). After the fourth week of drug treatment, there was no significant difference in the relative tumor volume of trametinib-treated tumors compared to trametinib plus I-BET151 treated tumors (Fig. 12A). The mice were sacrificed after four weeks of treatment, and tumors were resected.

Histological analysis of H\&E stained tumor sections were analyzed for increases in tumor necrosis, ischemia and fibrosis in all treatment groups. A statistically significant increase in focal tumor necrosis over the control was seen in the combinatorial treatment (Fig. 12B). Tumor necrosis was scored on a scale of zero to five; a representative example of tumor necrosis is shown in Figure 12D. Trametinib treatment resulted in regions of coagulative ischemic necrosis, which appeared to be abrogated by addition of I-BET151 (Fig. 12C \& E). Based on H\&E and Trichrome staining of the tumor sections, we observed that trametinib alone reduces fibrotic tissue in these tumors, while the combinatorial 
therapy increased the fibrotic content (Fig. 12F \& G). Because we do not have overall survival data for these animals, we cannot conclude whether these histological changes correlate with changes in the rates of metastatic disease progression. Based on the literature, increased tumor necrosis and fibrosis are indicative of treatment response. The implications of these changes in tumor patho-histology will be further explored in the discussion section of this chapter.

Based on the data shown in Figures 10 and 11, we hypothesized that dual inhibitor treatment would reduce the occurrence of liver and abdominal metastases. Upon resection, only one of the control animals presented with a metastasis to the liver (data not shown); a longer experiment would likely increase the incidence of metastasis to the liver. Four of the five control animals, however, presented with abdominal metastasis. Based on a binary "present" or "absent" metric, we calculated the percentage of animals presenting with abdominal metastases for each treatment group. Although not statistically significant, fewer animals that received the combinatorial treatment presented with abdominal metastases than any other treatment group (Fig. 12H). 
A.

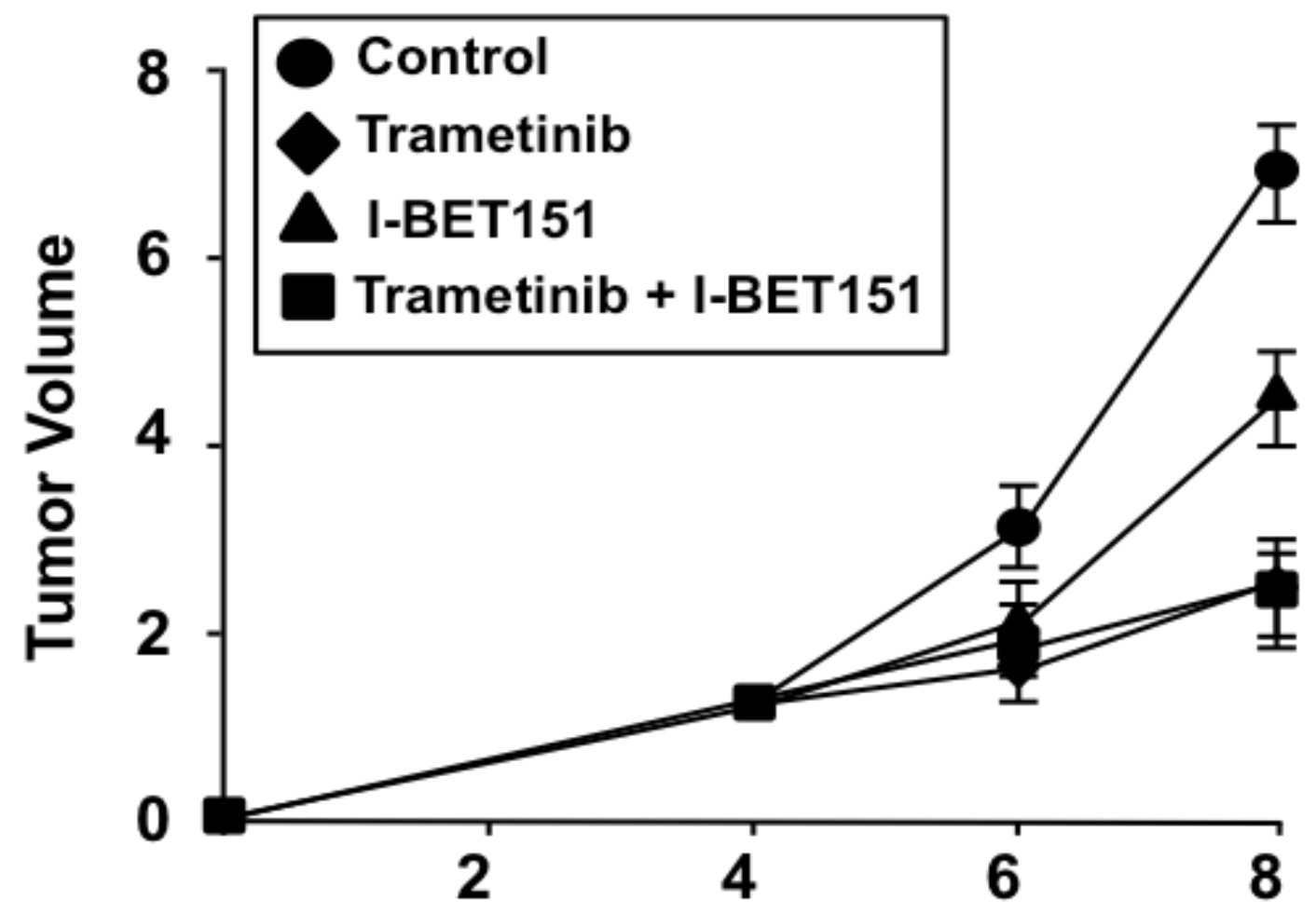

Time (weeks) 
B.

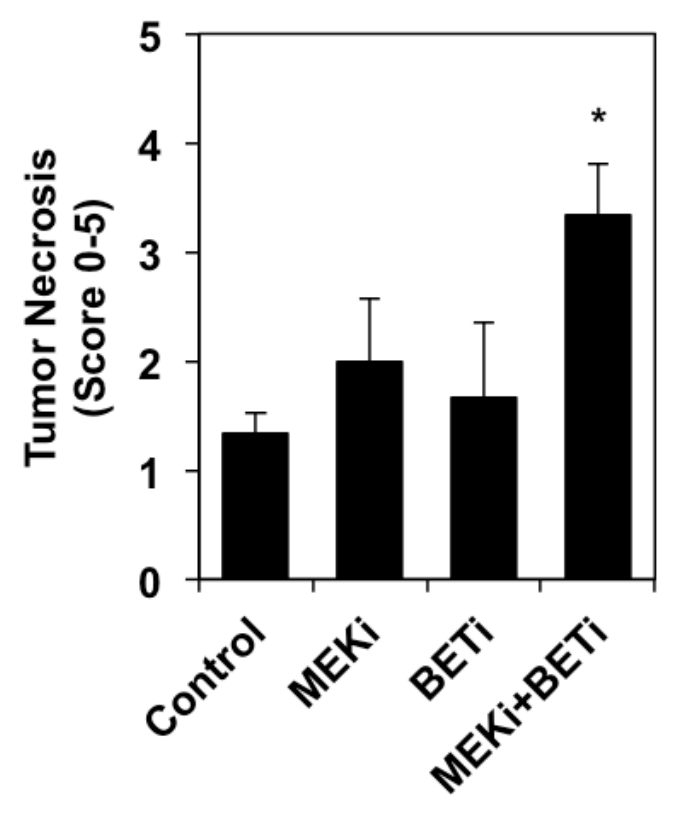

D.

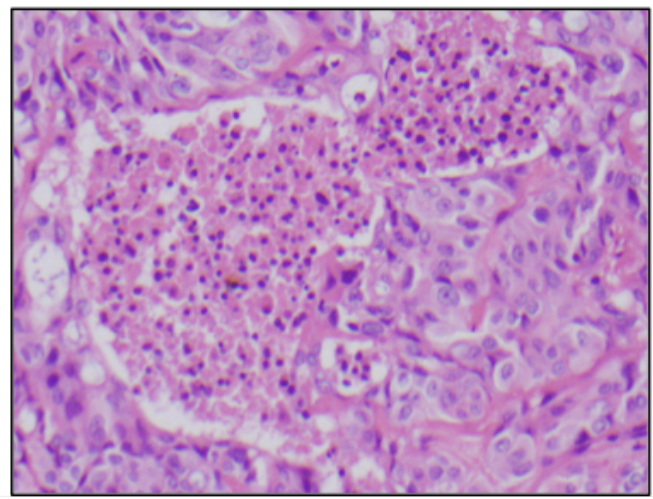

C.

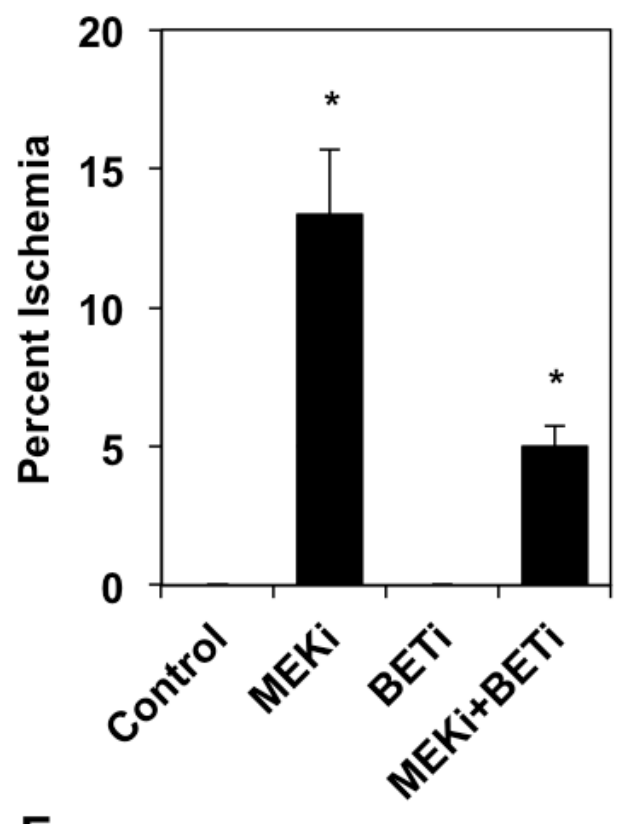

E.

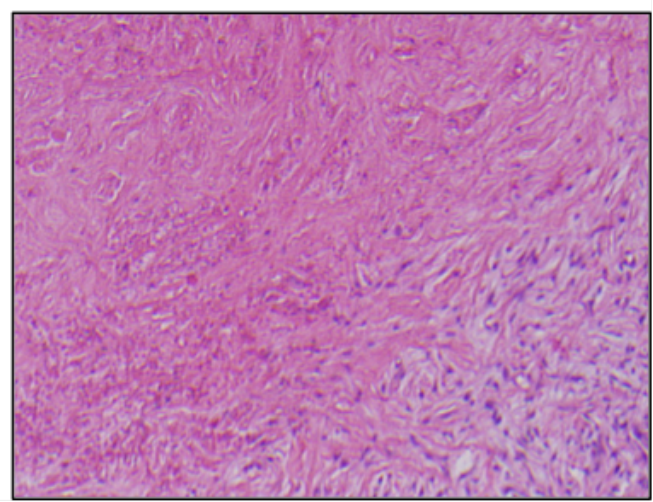




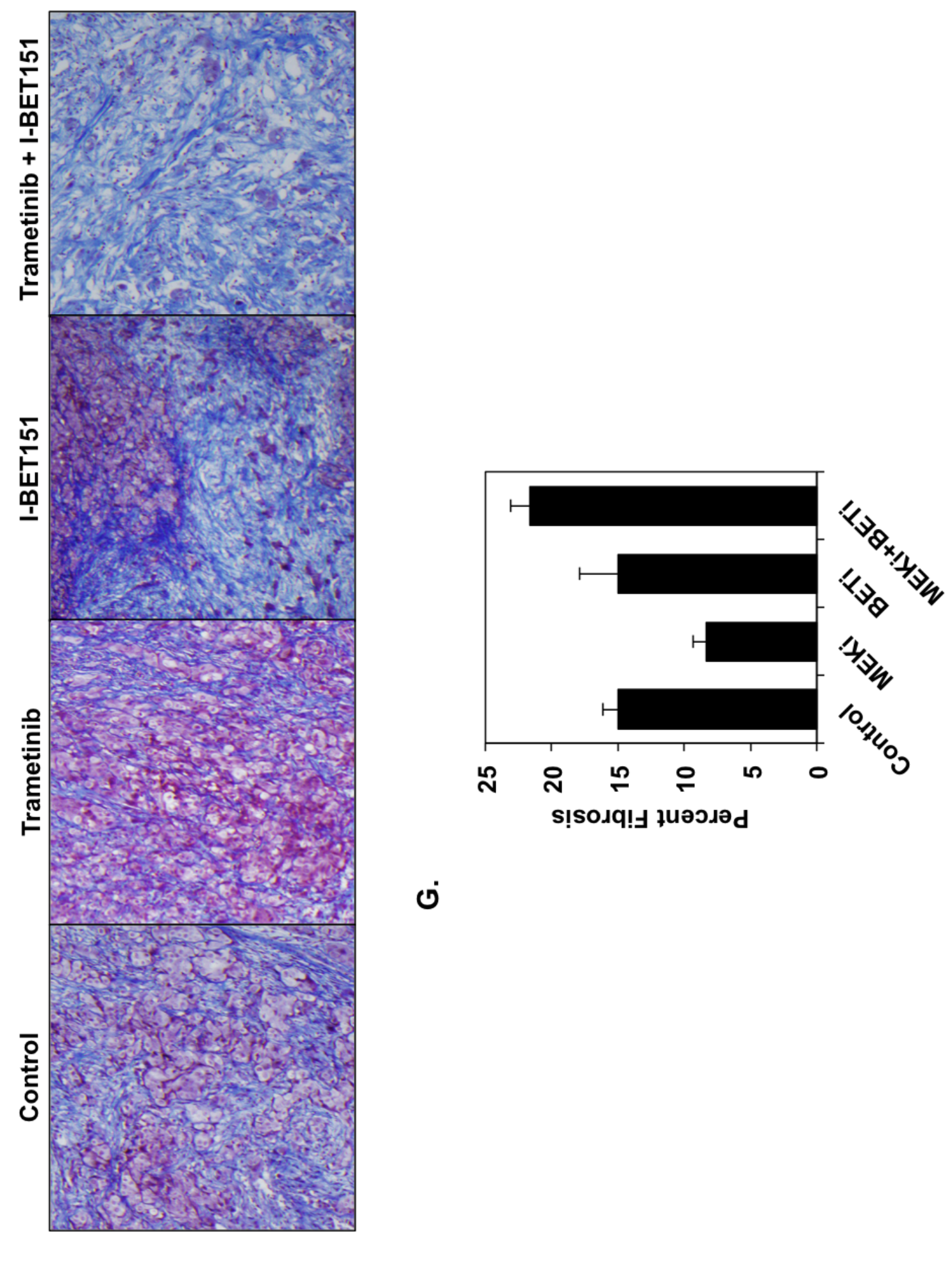


H.

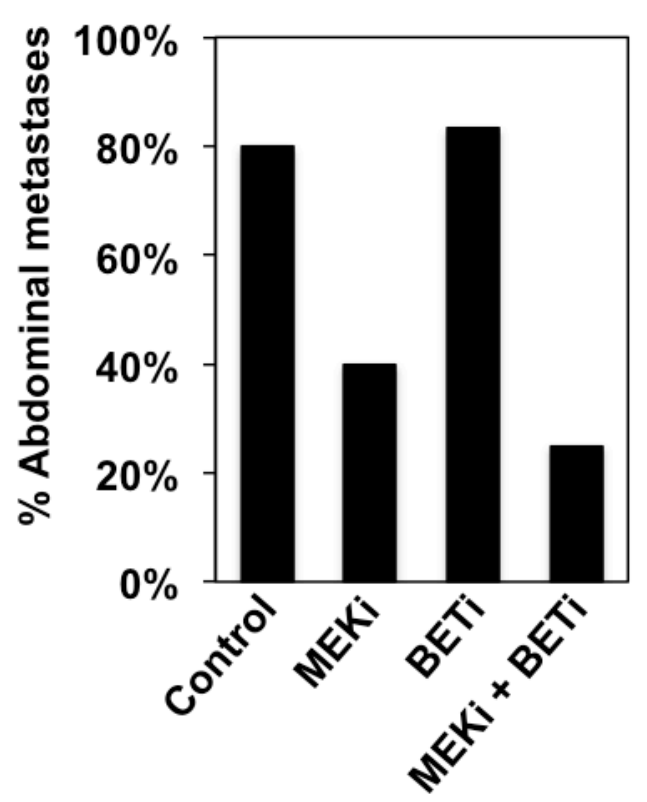


Figure 12: I-BET151 increases tumor necrosis in trametinib-treated orthotopic PDAC model. (A) Administration of I-BET151 with trametinib failed to further reduce the overall tumor volume of PDAC compared to trametinib alone, as detected by volumetric MRI. (B \& C) Quantification of pathohistological parameters based on H\&E stained PDAC tumors; (B) tumor necrosis scored on a scale of 0 to 5 ; (C) percent tumor ischemia. (D \& E) Representative images of (D) tumor necrosis and $(E)$ tumor ischemia. $(F)$ Representative examples of tumor fibrosis in each treatment group as highlighted by Trichrome staining. (G) Quantification of fibrosis in PDAC tumors under indicated treatment conditions. (H) The percentage of animals in each treatment group presenting with abdominal metastases; no significant difference. 


\section{DISCUSSION}

Pancreatic cancer is an aggressive, highly malignant disease for which there are too few efficacious therapies. New strategies are needed to counter the ability of this cancer to evade cell death and acquire drug resistance. Trametinib inhibition of MEK has shown promise in slowing the growth of tumors in pre-clinical xenograft mouse models, but was not shown to improve overall survival when paired with gemcitabine in a clinical trial (Infante et al., 2014). In this study we test the efficacy of a novel combination of inhibitors, trametinib and I-BET151, to induce apoptosis and reduce the population of CICs. In tumor spheres, trametinib and I-BET151 synergize to reduce cell counts further than either single agent alone. The loss of cell numbers is due to an increase in apoptotic signaling, as evidenced by a reduction in nuclear DNA content and an increase in cleaved caspase 3.

Individually, both inhibitors were able to increase cellular levels of the BIM protein. BIM levels, however, were highest in samples treated with the combinatorial therapy. Trametinib did not significantly induce BIM transcription, suggesting a posttranslational effect on the stability of BIM protein. ERK is known to phosphorylate BIM, thus targeting it for ubiquitin-mediated proteasomal degradation. Trametinib treatment reduced phosphorylation of BIM, and is likely stabilizing BIM levels by inhibiting activation of ERK. I-BET151, on the other hand, did preferentially increase $B I M_{L}$ and $B I M_{S}$ mRNA splice forms. It is unclear how I-BET151 activates transcription and alternative splicing of BIM. 
$B I M$ is transcriptionally regulated by numerous transcription factors in a dynamic manner: FOXO3a, E2F2, and CHOP-C transcriptionally upregulate BIM, while Myc, NFאB, cJUN, RUNX, and SMAD3 actively repress its transcription. In addition, $B I M$ is post-transcriptionally regulated by splicing factors and microRNAs (Sionov et al., 2015). BET proteins are typically associated with transcriptional activation and elongation of gene targets. Because we observed an increase in BIM mRNA expression following I-BET151 treatment, it is likely that BET proteins mark the enhancer of a repressor protein required to suppress basal BIM expression. Trametinib alone decreases Myc levels without a concomitant increase in BIM transcription, therefore I-BET151-mediated repression of $M y c$ is unlikely responsible for the activation of BIM. Preliminary experiments in the lab show no change in $\mathrm{FOXO} 3 a$ protein levels under any treatment condition (data not shown). Infection of 366 and 608 with adenovirus expressing the repressor of $\mathrm{NF} \kappa \mathrm{B}, \mathrm{I}_{\kappa \mathrm{B}} \alpha$, downregulates the $\mathrm{NF} \kappa \mathrm{B}$ target gene CXCL8. It does not, however, alter BIM transcription suggesting that inhibition of NFKB activity did not impact BIM expression in this cellular context (Fig. S2). Understanding the mechanisms contributing to the induction of $B I M_{L}$ and $B I M_{S}$ in I-BET151-treated PDAC would require further experimentation due to the complexity of BIM regulation.

Although I-BET151 increased BIM protein levels, this alone was no sufficient to stimulate apoptosis. Without trametinib treatment, BIM failed to accumulate at the mitochondria despite an abundance of the protein. This correlated with reduced mitochondrial accumulation of BAX and BAK, the 
proteins responsible for permeabilizing the mitochondrial outer membrane during apoptosis. As expected, trametinib facilitated apoptosis because ERK activation has been shown to contribute to numerous anti-apoptotic mechanisms in cancer (Deng et al., 2000; Xu et al., 2009). For example, ERK can stabilize BCL-2 via phosphorylation; increased BCL-2 levels sequester pro-apoptotic proteins BIM, BAX, and BAK and prevent their ability to induce apoptosis (Dai et al., 2013; Willimott and Wagner, 2010). Following the various treatments, immunoprecipitation of BCL-2 followed by immunoblotting for BIM, BAX, and BAK would reveal whether BCL-2 was sequestering the pro-apoptotic proteins in a drug-dependent manner. Although further downstream in the apoptotic pathway, ERK can also phosphorylate pro-caspase 9, preventing its assembly into the apoptosome and, thus, significantly dampening cell death mechanisms (Arlt et al., 2013).

KRAS-mutant pancreatic cancer cells have been shown to prevent mitochondrial apoptosis by influencing fragmentation of mitochondrial networks. ERK-dependent phosphorylation of DRP1 results in mitochondrial fission (Kashatus et al., 2015). Fragmentation of the mitochondria prevents the stable association of BAX with the mitochondria, thus inhibiting mitochondrial membrane permeabilization (Renault et al., 2015). In collaboration with the Dr. David Kashatus' laboratory (University of Virginia), we were able to further examine ERK-mediated mitochondrial fragmentation as a potential resistance mechanism to MEK inhibition in PDAC. We hypothesize that if DRP1-mediated mitochondrial fission is blocking the ability of I-BET151 to induce BIM-mediated 
apoptosis, then inhibition of DRP1 should prevent mitochondrial fission and allow I-BET151 to effectively induce cell death.

Immunofluorescent staining of mitochondria confirmed the fragmented phenotype in untreated 2D cell cultures. In this experiment, mitochondrial fusion was induced following the inhibition of MEK as previously reported (Kashatus et al., 2015; Prieto et al., 2016). Similar to MEK inhibition, the BET inhibitor also increased fusion of mitochondrial networks (Fig. S3). Unfortunately, the fragmented phenotype observed in untreated cells was lost when the cells were taken through $3 \mathrm{D}$ cultures suggesting that mitochondrial dynamics were sensitive to changes in signaling pathways utilized by multicellular spheroid cultures (data not shown). Lastly, stable shRNA knockdown of DRP1 in the pancreatic cancer cell line mPanc96 did not further induce apoptosis upon treatment with I-BET151 compared to the shRNA scramble cell line (Fig. S4). The results of these experiments suggest that unlike the synergistic induction of cell death produced by trametinib and I-BET151, the knockdown of DRP1 did not potentiate I-BET151 cell death mechanisms.

In addition to evaluating changes in mitochondrial dynamics and apoptosis, PDAC tumor spheres were examined for markers of autophagy following trametinib and I-BET151 treatment. As shown in Figure S5, we observed increased levels of autophagy makers LC3A/B upon I-BET151 treatment. Autophagy is a metabolic process that delivers macromolecules and organelles to lysosomes for degradation (Mukhopadhyay et al., 2014). BIM $\mathrm{L}_{\mathrm{L}}$ was found to support acidification of lysosomes that later associate with autophagic 
vesicles; it is possible that the upregulation of the BIM $\llcorner$ splice form by I-BET151 contributes to the increase of LC3A/B. Autophagy has complex roles in PDAC cancer metabolism and tumor progression. While some studies indicated that autophagy is tumor promoting, others have suggested that it is tumor suppressive (Gómez et al., 2015). In combination with gemcitabine, cannabinoid treatment results in ROS-dependent activation of the autophagic program and strongly inhibitors tumor growth in a PDAC xenograft model (Donadelli et al., 2011).

In addition to increasing cellular levels of BIM, the I-BET151 drug may be downregulating the expression of pro-apoptotic signaling proteins. Depletion of BRD2 has been shown to downregulate APAF1 and caspases 1, 7, and 9 . Conversely, overexpression of BRD2 induces apoptosis (Hnilicová et al., 2013). The translocation of BIM to the mitochondria precedes activation of APAF1 or caspase 9 during apoptosis, but we cannot rule out the effect of depleting these proteins upon treatment with I-BET151. Although the exact influence of each drug on apoptotic signaling remains unknown, our work indicates that the combination of trametinib and I-BET151 more effectively induces apoptosis in 366 and 608 tumor spheres than trametinib alone.

Importantly, we show that the combinatorial therapy has the capacity to reduce EMT and CIC makers, as well as impede migration in vitro. Trametinib treatment alone resulted in transcriptional activation of certain master-switch transcription factors and stem cell transcription factors. It would be interesting to evaluate the expression of EMT and $\mathrm{CIC}$ associated genes in chronically treated 
or trametinib-refractory tumors compared to trametinib-naïve tumors. Upregulation of these transcription factors may orchestrate trametinib-resistance mechanisms by promoting a more mesenchymal or stem-like phenotype. IBET151 was able to mitigate trametinib-induced expression of the EMT and CIC transcription factors. It is unclear as to whether the disruption of superenhancers with I-BET151 would prevent PDAC tumors from developing resistance to trametinib by impeding transcriptional reprogramming, but the preliminary data presented in this study support that hypothesis.

Phenotypically, the combination of trametinib and I-BET151 was able to reduce expression of mesenchymal markers Fibronectin and Vimentin in both 366 and 608 cell lines. In the 608 cell line, the combinatorial therapy also reduces E-cadherin protein levels. BET inhibition in an EMT model of NSCLC results in silencing of the E-cadherin gene $\mathrm{CDH} 1$ (shown in the next chapter), and could explain this result. Scratch assays show that the BET inhibitor alone may actually increase migration, which could be due to the loss of E-cadherin. The inhibition of E-cadherin is considered a "side-effect" of I-BET151 treatment in our model system and could deleteriously impact PDAC tumor progression in vivo. This observation underscores the importance of testing I-BET151 in preclinical models. In combination with trametinib, however, I-BET151 significantly slows migration over time. The acquisition of migratory and invasive phenotypes is a rate-limiting step of metastasis (Liang et al., 2007; Valastyan and Weinberg, 2011). Based on these results, we postulate that the combinatorial therapy will 
constrain metastatic disease in vivo. Further investigation of the effect of trametinib + I-BET151 on invasion, migration and metastasis are warranted.

The BET inhibitor JQ1 has been previously been shown to suppress tumor growth in a PDX model of PDAC, however this is the first study to show that the I-BET151 compound also has the ability to significantly slow tumor growth in a PDX model of PDAC (Garcia et al., 2016). Although we observed no change in tumor volume between trametinib alone and trametinib + I-BET151 treated tumors, the content of the tumor masses was different between treatment groups. The combinatorial treatment group was the only group to show a significant increase in focal tumor necrosis over the control group (Fig. 12B). Naïve PDAC tumors often show areas of base-line necrosis, which is an indicator of poor prognosis because the tumor is aggressively outgrowing its nutrient supply (Hartman and Krasinskas, 2012a; Le Scodan et al., 2008). However, focal necrosis is one of the best clinical indicators of adjuvant-induced tumor regression (Stremitzer et al., 2015; Verbeke et al., 2015).

We also noticed that trametinib treatment alone contributed to marked areas of coagulative necrosis, indicative of ischemia (Fig. 12D). This is consistent with studies showing that the MEK/ERK pathway is pro-angiogenic, and that inhibition of MEK promotes intra-tumoral ischemia (Bridgeman et al., 2016; Chamorro-Jorganes et al., 2016; Sun et al., 2015; Wang et al., 2016). The addition of I-BET151 to trametinib reduced the incidence and extent of ischemic regions, offering an unexpected therapeutic effect of BET inhibition in vivo. Antiangiogenic compounds such as trametinib can be therapeutically 
counterproductive for a variety of reasons: (1) limitation of drug delivery to the tumor, (2) induction of hypoxia-mediated metastasis and drug resistance, (3) immunosuppression (Blanco et al., 2015; Chand et al., 2016; Imaizumi et al., 2016; Maione et al., 2012). Furthermore, PDAC is largely insensitive to antiangiogenic drugs, such as VEGF inhibitors (Jayson et al., 2016). MEK inhibition, however, has implications for tumor biology in addition to anti-angiogenesis. In this model, it is unclear whether it is therapeutically beneficial to eliminate the nutrient and oxygen supply to the tumor in the context of MEK inhibition.

According to the guidelines for histologic grading of treatment response put forth by Hartman and Krasinskas, an increase in fibrotic mass or stroma is indicative of a treatment response in PDAC (Hartman and Krasinskas, 2012b). In fact, fibrosis is considered "the predominant feature of tumor regression" in pancreatic cancer (Verbeke et al., 2015). Irradiated pancreatic tumors often demonstrate replacement of tumor with fibrosis rather than shrinkage of the mass (White et al., 2005). An increase in the number of tumor-associated fibroblasts has also been shown to restrain tumor growth (Neesse et al., 2015). It is important to emphasize that the observed increase in fibrosis following combinatorial trametinib and I-BE151 treatment in our in vivo experiment was modest. Based on the overall histological responses, it is likely that the combination of trametinib and I-BET151 would provide an overall survival benefit, but we would need to confirm this experimentally using a patient-derived orthotopic PDAC model. 


\section{FUTURE DIRECTIONS}

\section{Combinatorial therapy increases overall survival in an in vivo xenograft model}

Our in vivo xenograft experiments comparing treatment effect on tumor volume showed that I-BET151 did not increase the efficacy of trametinib in preventing tumor growth (Fig. 12A). However, we were able to show that the histopathology of the tumors was different in animals treated with the combination therapy (Fig. 12B-G). Based on these observations, we predict that the increase in necrosis and fibrosis seen with the combinatorial treated mouse tumors is consistent with tumor regression. If true, the combinatorial treatment should confer an overall survival advantage. To test this hypothesis, we would perform additional in vivo xenograft experiments using overall survival as the primary endpoint rather than tumor volume. Experiments would be designed such that the starting material for the tumor explants were smaller to allow for enough time to observe metastases in the untreated controls. Our predictions using such a model would be that I-BET151 would improve the effectiveness of trametinib-treated animals with regard to overall tumor volume and metastasis.

Similar to our preliminary experiment, 366 and 608 tumors would be implanted onto the pancreata of 40 mice per tumor type, this time using 10 animals per treatment group to increase statistical power. The tumors would be permitted to grow for four weeks before doing a preliminary MRI and initiating treatment with a vehicle ( $0.5 \%$ hydroxypropyl methylcellulose in $0.1 \%$ Tween 80$)$, trametinib alone (1 mg/kg), I-BET151 alone (25 mg/kg), or the combination of 
trametinib and I-BET151. Drugs would be administered daily by oral gavage. Every 7 days following the initiation of treatment, volumetric MRI would be used to track tumor growth over time. Mice would be sacrificed when tumor volume exceeds $1500 \mathrm{~mm}^{3}$ in order to limit primary tumor burden. Survival would be plotted over time to determine which treatment confers the greatest duration of survival. We predict the dual inhibitor treated mice would receive an overall survival benefit compared to the single agent trametinib treated mice.

In our preliminary mouse experiment for this study, only one animal presented with liver metastases (data not shown), however this model has previously resulted in metastases to the liver following 8 weeks of tumor growth (Walters et al., 2013). If we are able to perform the proposed overall survival xenograft experiment described above, we would also examine the mice for liver and abdominal metastases. Based on the trend suggested by our initial mouse experiment (Fig. 12H), we hypothesize that mice treated with the combinatorial therapy would present with fewer metastases as compared to either single agent treated animal.

Such experiments would provide us with additional information about the efficacy of this drug combination in established PDAC. If successful in extending overall survival in our xenograft mouse model, this drug combination would be a worthwhile therapeutic approach to test in a clinical setting of patients who present with unresectable late-stage PDAC. Both MEK and BET inhibitors have shown safety in clinical trials, however, the safety of the combination would need to be evaluated in humans. Pertinent to the treatment of PDAC, is the efficacy of 
these treatments in combination with or sequentially following Gemcitabine treatment, the current standard-of-care for patients with unresectable tumors.

\section{Combinatorial therapy reduces outgrowth in an occult metastatic tumor model}

As shown in Figures 10 and 11, dual therapy inhibits mesenchymal phenotypes, reduces the $\mathrm{CIC}$ population and diminishes the ability of these cells to migrate in vitro. While the combination therapy did not reduce tumor volume better than trametinib alone, mice that received the dual treatment trended toward fewer abdominal metastases compared to either single agent alone (Fig. 12H). Based on these positive results, additional experiments are warranted to conclusively evaluate the efficacy of dual therapy on preventing metastatic outgrowth of PDAC. In addition to examining animals for abdominal and liver metastases in the xenograft experiment described above, it would be beneficial to perform experiments that measure the effects of these drugs in an occult metastasis model. This experiment is relevant in modeling recurrence of disease in patients who have had their tumors surgically resected.

To perform this experiment, we would use previously established 366 and 608 tumor cell lines that have been lentivirally transduced to express firefly luciferase. Again, ten mice would be used per treatment group for each cell line. The 366 and 608 cells would be taken through tumor sphere culture to enrich for $\mathrm{CIC}$ before being enzymatically dissociated and counted. Using a surgical left flank incision to access the spleen, $1 \times 10^{6}$ cells suspended in $50 \mu \mathrm{L}$ of serumfree media would be injected into the spleens of eight week old athymic nude 
mice. Ten minutes post-injection, the spleen would be resected and the incision site will be sutured closed. As previously shown using this protocol, the cells will circulate directly to the liver to establish microscopic liver metastases during this ten-minute incubation period (Newhook et al., 2016).

Bioluminescent imaging would be used to measure the growth of liver metastases over time. The first round of imaging would take place 48 hours postinjection, and would be used base-line luminescent values. Immediately following this baseline reading of micrometastases, drug treatment would begin. Treatment with a vehicle $(0.5 \%$ hydroxypropyl methylcellulose in $0.1 \%$ Tween 80), trametinib alone (1 mg/kg), I-BET151 alone $(25 \mathrm{mg} / \mathrm{kg})$, or the combination of trametinib and I-BET151 would be administered daily by oral gavage. Radiance of the hepatic metastases would be measured every 7 days, and the data would be evaluated for time-to-progression, growth kinetics, and overall survival.

In this occult tumor model, our collaborators have already shown that trametinib significantly extends time-to-progression and overall survival compared to vehicle control. This proposed experiment, however, differs from the published study in tumor spheres are enriched for CICs, which would be predicted to impact survival following splenic injections. This would be predicted to expedite the kinetics of hepatic tumorigenesis by increasing the number of cells able to establish micrometastases. Regardless, we still expect that the addition of a BET inhibitor to trametinib would further delay outgrowth of tumor cells compared to trametinib alone. We also expect the combination will provide 
an extension in overall survival compared to either single agent alone. To our knowledge, no one has tested the BET inhibitor alone in an occult model of PDAC. Due to the ability of the BET inhibitor to reduce the population of CICs in tumor sphere culture (Figure 11), we hypothesize I-BET151 will confer an overall survival benefit to the animals compared to vehicle control.

Roughly $80 \%$ of patients who undergo surgical resection of their PDAC tumors will have recurrent disease (Dimastromatteo et al., 2015; White et al., 2005). This is postulated to be due to the outgrowth of micrometastases that has already occurred prior to surgical resection. Thus effective adjuvant and neoadjuvant therapy is wanting. If effective in this in vivo model of occult PDAC metastasis, it would be reasonable to pursue additional preclinical and clinical testing of this combination in an adjuvant and neoadjuvant setting. Further preclinical studies could evaluate the combination or sequential treatment of the MEK and BET inhibitors with Gemcitabine, the current standard-of-care for adjuvant PDAC patients. 
CHAPTER 4: BET INHIBITION IMPEDES MIGRATION AND INVASION PHENOTYPES IN NON-SMALL CELL LUNG CANCER 


\section{INTRODUCTION}

\section{Non-Small Cell Lung Cancer}

Lung cancer is the most commonly diagnosed cancer in the world. In the US, lung cancer is the leading cause of cancer-related mortality accounting for roughly one in every four cancer deaths (Cufer et al., 2013; David et al., 2016). The American Cancer Society predicts 224,390 new lung cancer diagnoses in 2016 and 158,080 lung cancer-related deaths. In fact, lung cancer contributes to more deaths than breast, colorectal, and prostate malignancies combined (American Cancer Society, 2016). Lung malignancies are categorized into two basic types based on histology: non-small cell lung cancer (NSCLC) comprises approximately $85 \%$ of cases, and small-cell lung cancer (SCLC) makes up the remaining 15\% (Hamilton et al., 2016).

Non-small cell lung cancer (NSCLC) is further delineated into three histological subtypes: adenocarcinoma, squamous cell carcinoma, and large cell carcinoma. Adenocarcinomas arise from glandular secretory cells in the lung; this is the most commonly diagnosed NSCLC subtype, accounting for roughly $40 \%$ of cases (Aisner and Marshall, 2012). Squamous cell carcinoma (SCC) originates from the squamous epithelial cell layer that lines the bronchial tubes of the lungs. SCC accounts for approximately $25 \%$ of NSCLC diagnoses (Kim et al., 2012). Large cell lung carcinomas arise from epithelial cells within the lung and are characterized as being undifferentiated and lacking cytological or architectural features of other lung cancer subtypes (Travis et al., 2011). 


\section{Risk Factors and Prognosis for Non-Small Cell Lung Cancer}

Smoking tobacco is the primary risk factor for NSCLC and has been linked to roughly $80-90 \%$ of cases. While smoking rates in the US are dropping as a result of anti-smoking campaigns, smoking rates remain high in much of the developing world. Mutations contributing to NSCLC tumorigenesis can also be triggered by occupational and environmental carcinogens such as asbestos, radon, polycyclic aromatic hydrocarbons, arsenic, and nickel, among others (Aprea et al., 2016; David et al., 2016; Mattei et al., 2016; Noh et al., 2016). In more rare instances, NSCLC can be initiated by viral infections including the Epstein-Barr virus (EBV) and Human papillomaviruses (HPV) (Brouchet et al., 2005; Chang et al., 2015; Fang et al., 2015; Sarchianaki et al., 2014).

Early detection of malignancy can drastically improve a patient's prognosis. The five-year survival rate for localized NSCLC is at $52 \%$, versus $5 \%$ for metastatic disease (Travis et al., 2011). Studies have shown that screening high-risk patients using a low dose-CT scan provides a $20 \%$ reduction in lung cancer-associated mortality (Bach et al., 2012; National Lung Screening Trial Research Team et al., 2011). If diagnosed at an early stage, patients undergo surgical resection of their tumors followed by adjuvant radiation and/or chemotherapy. Unfortunately, over half of patients present with Stage IV metastatic disease and are faced with a median survival time below 18 months (Mirsadraee et al., 2012). Metastatic NSCLC is inoperable and largely insensitive to chemotherapy and radiotherapy. Patients with treatmentresponsive tumors will generally relapse with disease due to acquired resistance. 
The well-documented insensitivity to therapy of NSCLC highlights the need to identify targetable molecular pathways that can lead to prolonged disease remission.

\section{Molecular Signatures and Targeted Therapy for NSCLC}

One of the best characterized and targetable oncogenes in NSCLC is the epidermal growth factor receptor (EGFR) tyrosine kinase. According to The Cancer Genome Atlas, EGFR is amplified or overexpressed in roughly $20 \%$ of adenocarcinoma cases (Kim et al., 2015). Additionally, approximately 15\% of NSCLC harbor activating mutations in the EGFR kinase domain, resulting in ligand-independent activation of EGFR. The two most common mutations are an in-frame deletion in exon 19 and a point mutation in exon 21 (L858R), both within the kinase domain (Maione et al., 2016; Sweis et al., 2016). Constitutive activation of EGFR kinase activity results in the hyperactivation of downstream pathways that control cell proliferation. Activating pathways in NSCLC include Akt, C-Jun N-terminal kinase (JNK), and mitogen-activated protein kinase (MAPK) (Raparia et al., 2013).

These genetic lesions can also be referred to as "sensitizing" mutations because they accurately predict patient sensitivity to targeted molecular therapies. When applied to identified NSCLC cohorts, therapies that block constitutively active EGFR yield significantly improved response rates compared to conventional chemotherapy (Aisner and Marshall, 2012). Gefitinib and erlotinib, two small molecule inhibitors that target the tyrosine kinase domain of EGFR, have been approved for use in NSCLC. Treatment with these tyrosine 
kinase inhibitors (TKIs) prolongs progression-free survival (PFS) over platinumbased chemotherapy in patients whose tumors harbor EGFR activating mutations. Importantly, patients treated with EGFR-TKIs also demonstrated enhanced quality of life and reduced treatment-induced toxicities compared to patients who received chemotherapy. Tumors without EGFR alterations are generally refractory to these EGFR-antagonists; this emphasizes the need to molecularly characterize tumors before selecting a patient's treatment options (Gatzemeier et al., 2007). Unfortunately, resistance to EGFR-TKIs often emerges within twelve months and researchers are now focused on identifying strategies to counter these resistance mechanisms (Sequist et al., 2011). Secondary mutations in EGFR, KRAS and MET have all been linked with resistance to EGFR-TKIs (Eberlein et al., 2015).

KRAS mutations occur in $25-30 \%$ of adenocarcinomas and are strongly associated with tobacco smoking (Aisner and Marshall, 2012; Naidoo and Drilon, 2016). As discussed in the previous chapter, mutant KRAS is an elusive target; no therapeutic agent targeting KRAS has been approved for NSCLC (Bhattacharya et al., 2015). Inhibition of proteins that function downstream of KRAS, including MEK and PI3K, have shown promise as targeted therapies for patients bearing tumors with mutant KRAS. In phase II clinical trials, MEK inhibition with selumetinib combined with docetaxel doubled PFS and OS compared to docetaxel alone (Kempf et al., 2016; Naidoo and Drilon, 2016). Resistance to MEK inhibition in KRAS mutant NSCLC can be mediated by 
transcriptional upregulation of ERBB3, and targeting EGFR in combination with MEK can inhibit this ERBB3-mediated drug resistance (Sun et al., 2014).

\section{A Rationale for Targeting EMT in NSCLC}

Mutations in KRAS and EGFR drive proliferative and survival signaling in NSCLC, but they can also give rise to metastatic disease. It bears repeating that localized disease is far more survivable than locally advanced or metastatic NSCLC. Elucidating strategies to target the spread of lung cancer are important to extending PFS and OS in patients. As explained in Chapter 1, EMT is a process that allows cancer cells to become more mesenchymal, invasive and migratory. In collaboration with the Bekiranov lab (University of Virginia), our lab has shown that the EMT process relies on vast genetic and epigenetic changes. During the EMT switch, epigenetic changes occur at gene bodies as well as promoters and enhancers, influencing numerous pathways critical for the induction of EMT. Various transcription factors, including NF-kB, AP-1 family members, and Myc were shown to be involved in chromatin-mediated transcriptional feedback loops important for the sustained activation of crucial EMT genes (Cieślik et al., 2013).

As explained in Chapter 1, BRD4 has been shown to facilitate the interaction of transcriptional machinery with active marks on chromatin at enhancers and promoters. We hypothesize that the displacement of BRD4 from the chromatin will disrupt important epigenetic mechanisms required for EMT. To test this hypothesis, experiments were performed to examine the effects of BET inhibitor JQ1 on EMT-associated markers and phenotypes. Importantly, we 
concluded that JQ1 downregulates expression of EMT-associated genes and impedes migration and invasion phenotypes. Although preliminary, these results suggest that BET inhibition may be therapeutically significant in disrupting NSCLC metastasis.

\section{RESULTS}

JQ1 inhibits expression of EMT master-switch transcription factors in an EMT NSCLC model.

We wanted to assess the effects of JQ1 on EMT in NSCLC. Preliminary experiments demonstrated that JQ1 impacts the expression of EMT protein markers in A549 tumor spheres treated with EMT-inducing cytokines TGF $\beta$ and TNF for 96 hours. Specifically, JQ1 reduces baseline levels of fibronectin, a mesenchymal marker, in cytokine-naïve tumor spheres. Upon stimulation with cytokines, fibronectin was induced in JQ1 treated tumor spheres, but not to the same level as JQ1-untreated tumor spheres (Fig. 13A). Regulation of mesenchymal markers Vimentin and N-cadherin did not appear to be affected by JQ1 treatment. E-cadherin levels were reduced by cytokine treatment, as well as JQ1 treatment (Fig. 13A). BRD4 is typically associated with activation of genes, so it follows that inhibition of BRD4 with JQ1 would have repressive transcriptional effects. To determine if the loss of E-cadherin following JQ1 treatment was due to loss of transcriptional activity, we performed qRT-PCR for mRNA expression levels of the E-cadherin gene, $C D H 1$. As expected, JQ1 results in significant inhibition of $\mathrm{CDH1}$ transcription (Fig. 13B). The addition of cytokines to JQ1 treatment further silences $C D H 1$ gene expression. 
During EMT, $C D H 1$ expression is modulated by transcription factors Snail, Slug, and Twist. Additionally, JQ1 has previously been shown to inhibit the activity of NF-KB, a transcription factor that upregulates expression of TWIST1 (Zou et al., 2014). For these reasons, we hypothesized that JQ1 was influencing the expression of the EMT master-switch transcription factors. Upon cytokine treatment, 3D A549 cultures upregulate expression of ZEB2, TWIST1, SNAI1 and SNAI2 (Fig. 13C). The effect of JQ1 on the expression of these genes varies. This cytokine-induced expression of ZEB2 is inhibited by JQ1 in a dosedependent manner. Without cytokine stimulation, baseline expression of TWIST1 is reduced by JQ1. Addition of the cytokines to JQ1 treated tumor spheres fails to induce expression of TWIST1. SNAI1 and SNAI2 appear to be induced by JQ1 in the absence of cytokines, however JQ1 also appears to dampen the cytokine-induced expression of these genes. These results demonstrate the ability of JQ1 to repress the expression of the EMT masterswitch transcription factors in a cytokine-induced tumor sphere model of NSCLC. 
A.

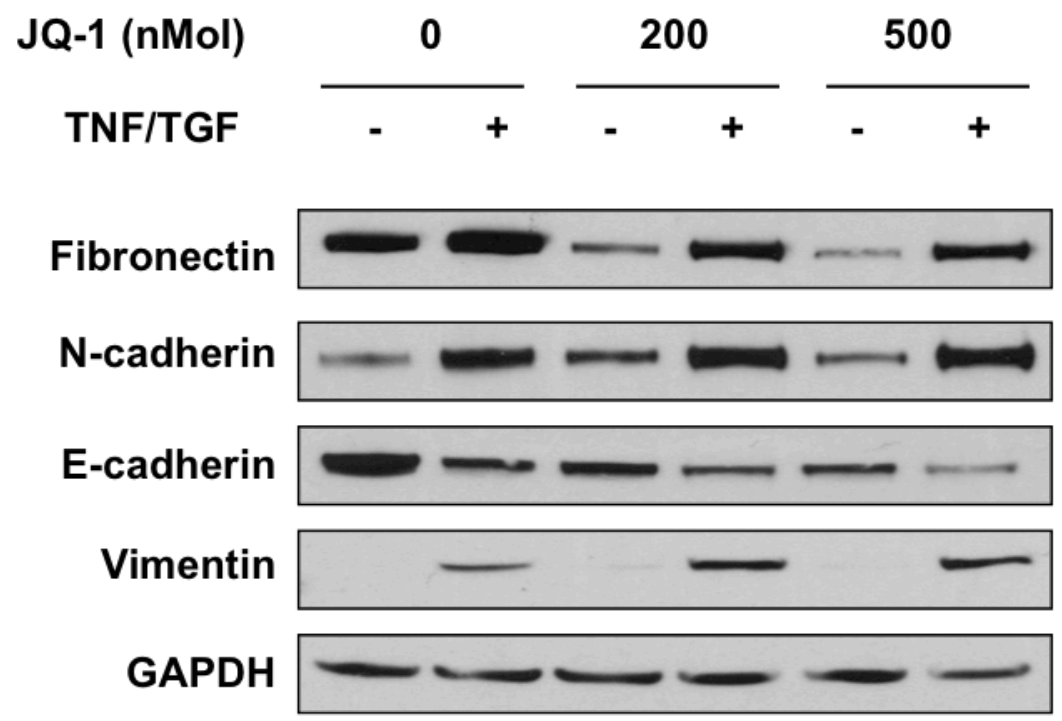

B.

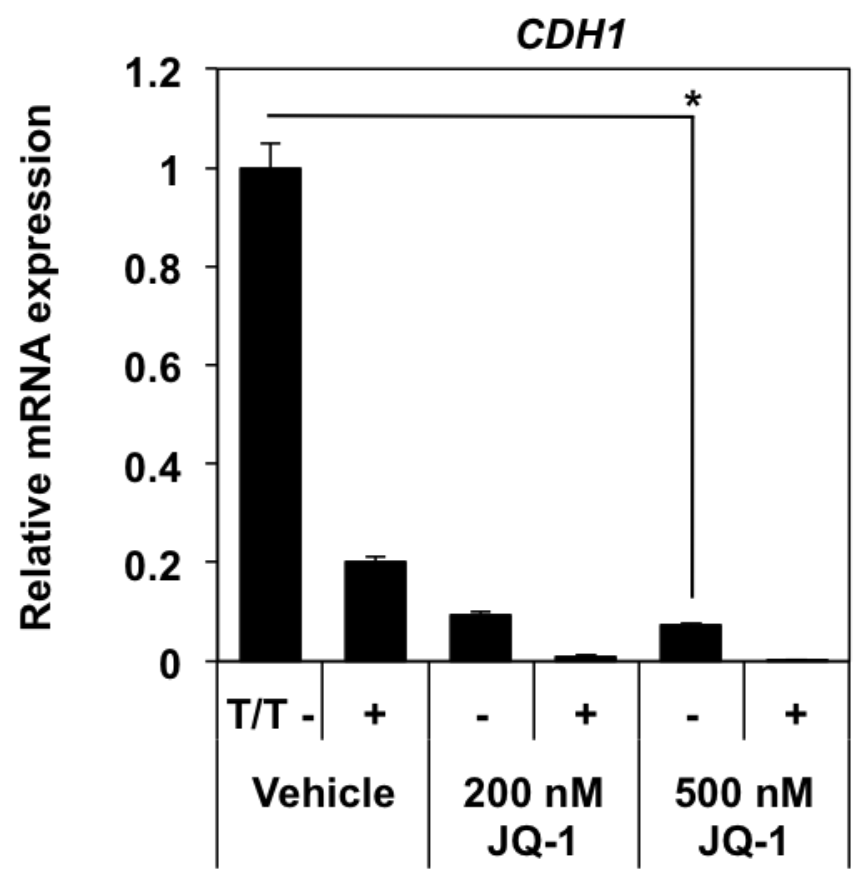


c.
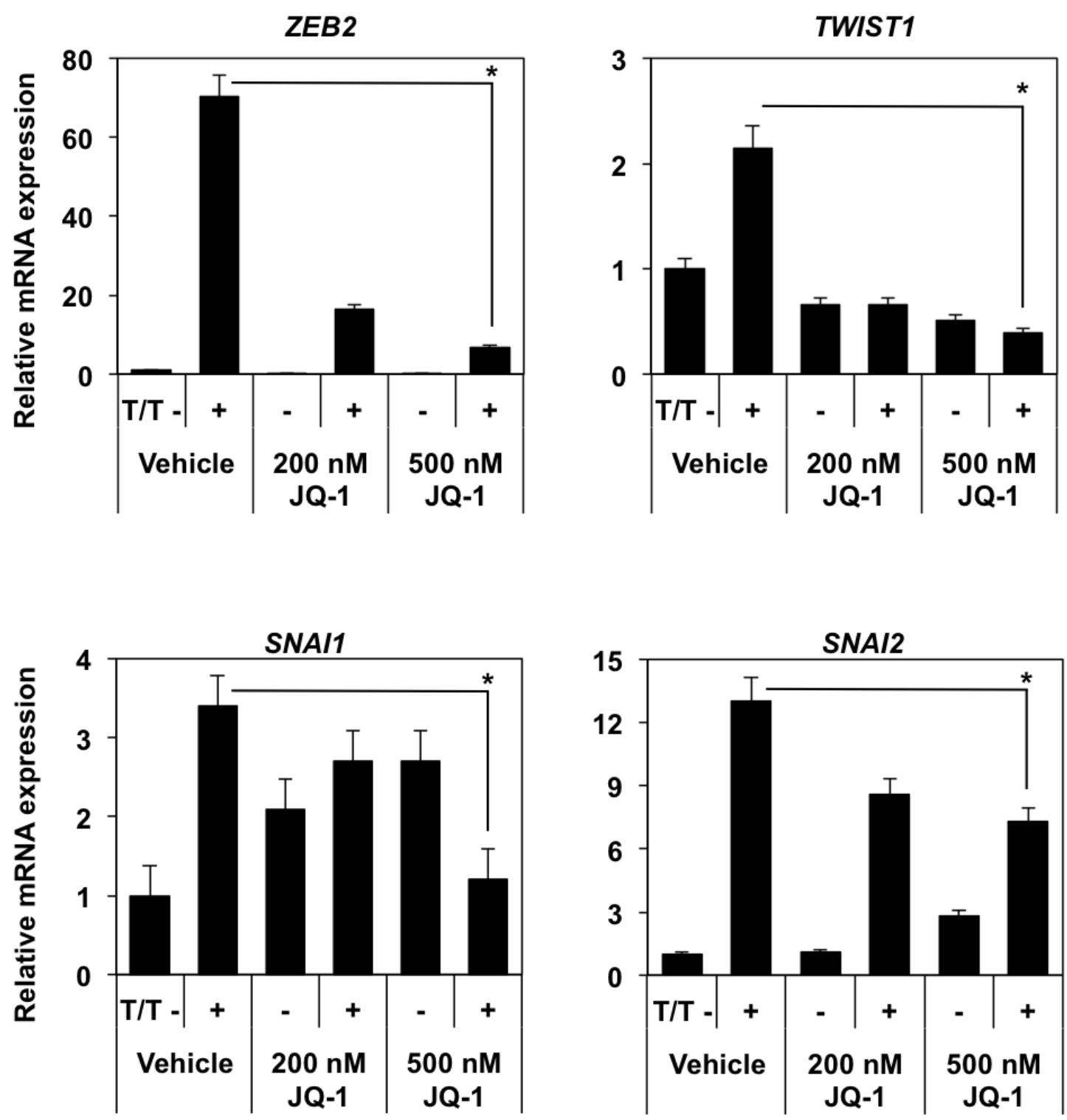
Figure 13. JQ1 inhibits expression of EMT master-switch transcription factors in TNF/TGF $\beta$-stimulated tumor sphere NSCLC cultures. (A) Immunoblot of EMT markers in tumor sphere A549 cultures treated in the presence or absence of JQ1 and the cytokines TGF $\beta$ and TNF. (B) qRT-PCR for CDH1 mRNA expression measured in tumor sphere A549 treated with or without JQ1 and TGF $\beta$ + TNF; normalized to GAPDH. (C) qRT-PCR for EMT masterswitch transcription factors in tumor sphere A549 treated with or without JQ1 and TGF $\beta+$ TNF; normalized to GAPDH. 


\section{JQ1 inhibits the invasive and migratory capacity of cytokine treated A549}

\section{tumor spheres.}

A hallmark of mesenchymal cells is their ability to migrate and invade. These functions are critical to the ability of malignant cells to leave the primary tumor, intravasate into the circulatory system, and spread to distal sites to establish metastatic tumors (Hamilton et al., 2016; Neri et al., 2011). The role of matrix metalloproteinases (MMPs) in the process of metastatic progression is well studied (Bae et al., 2013; Fuxe et al., 2010). A drug that can repress expression of MMPs and block migratory and invasive phenotypes would be therapuetically beneficial in the treatment of NSCLC. To this end, we sought to test the ability of JQ1 to prevent the strong cytokine-induced upregulation of MMP genes in tumor sphere A549 cultures. Expression of cytokine-induced MMP1 and MMP2 were significantly reduced by the $500 \mathrm{nM}$ treatment of JQ1. The expression of MMP3 and MMP10 following cytokine treatment was reduced by JQ1 in a dose-dependent manner (Fig. 14A).

Next we measured the effect of JQ1 on migration and invasion using transwell assays. A549 cells were taken through 3D tumor sphere culture and stimulated to undergo EMT with cytokines in the presence or absence of JQ1. These cells were dissociated and live cells were counted using trypan blue dye exclusion. An equal number of cells were plated into the top of each transwell in media lacking serum, and a chemoattractant of media containing 10\% FBS was loaded into the bottom of each transwell. Cells that were able to migrate or invade through each transwell were counted. In the migration assay, cells are 
plated on a porous membrane lacking extracellular matrix, and cell migration through the pores was measured following an 8 hour incubation. In the invasion assay, cells are plated on a matrix of Matrigel that mimics the composition of a basement membrane. In this assay, cells must acquire the ability to degrade the extracellular matrix and migrate through the transwell chamber during a 24 hour incubation.

As expected, the migratory capacity of A549 cells was increased by treatment with TGF $\beta$ and TNF (Fig. 14B and 14C). JQ1 impaired the ablity of "epithelial-like" cells (cytokine untreated) to migate in a dose-dependent manner. More importantly, the number of migratory cells in the cytokine-stimulated cultures that were treated with JQ1 was significantly lower than in the cytokinestimulated cultures that did not receive JQ1. Interestingly, the JQ1-treated cells that had been cytokine-stimulated to undergo EMT were less migratory than JQ1 cells that did not receive cytokine treatment. Similar results were observed in the invasion assay (Fig. 14D and 14E). Consistent with the idea that EMT induces more invasive phenotypes, cells that received cytokine stimulation were almost twice as invasive as unstimulated cells. Cytokine-naïve cells treated with JQ1 were less invasive than untreated cytokine-naïve cells. Additionally, JQ1 blocked the cytokine-induced invasiveness of these cells. Together, these results demonstrate the capacity of JQ1 to block invasion and migration in EMT-induced NSCLC cells. 
A.
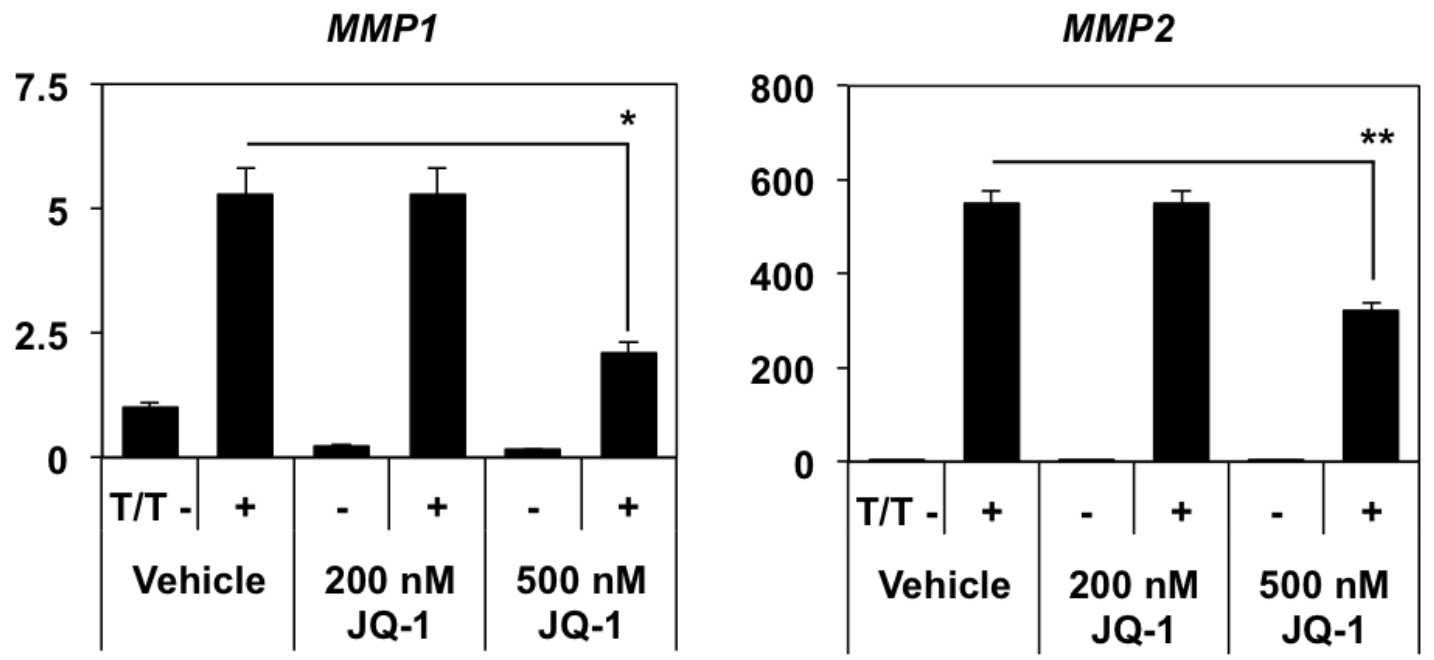

MMP3
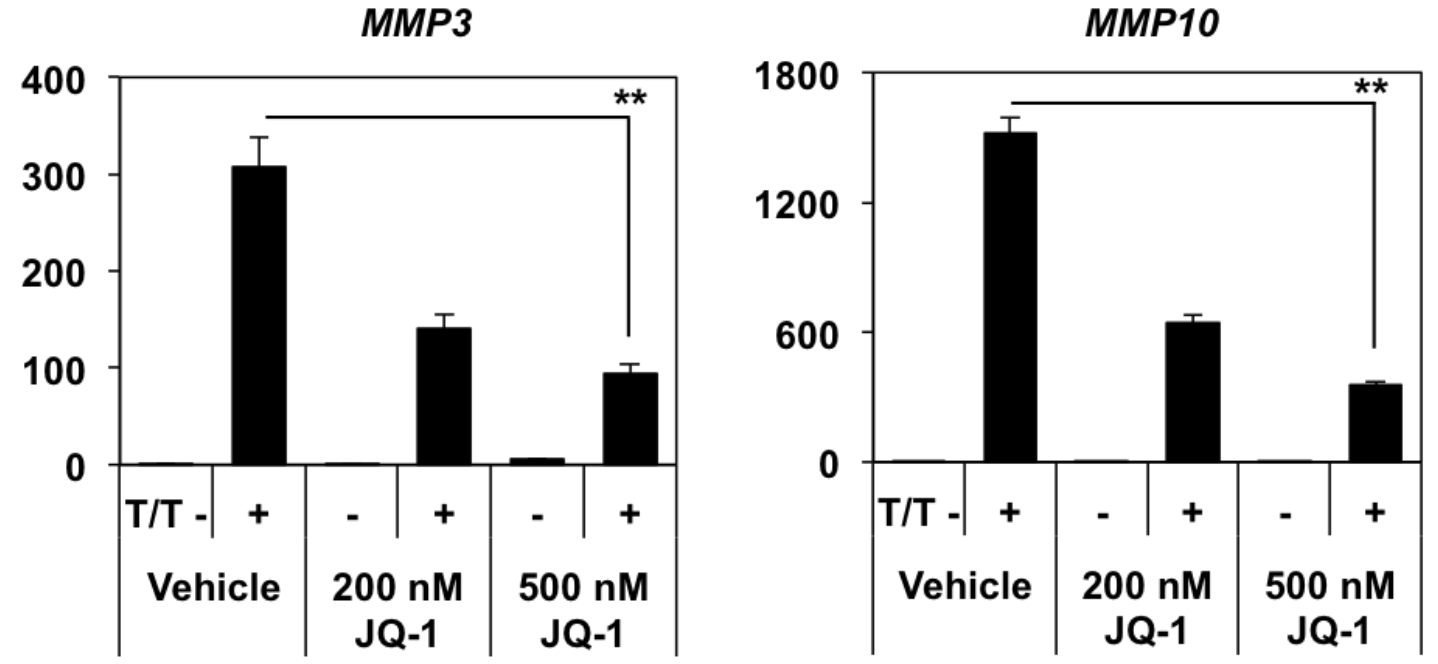
B.
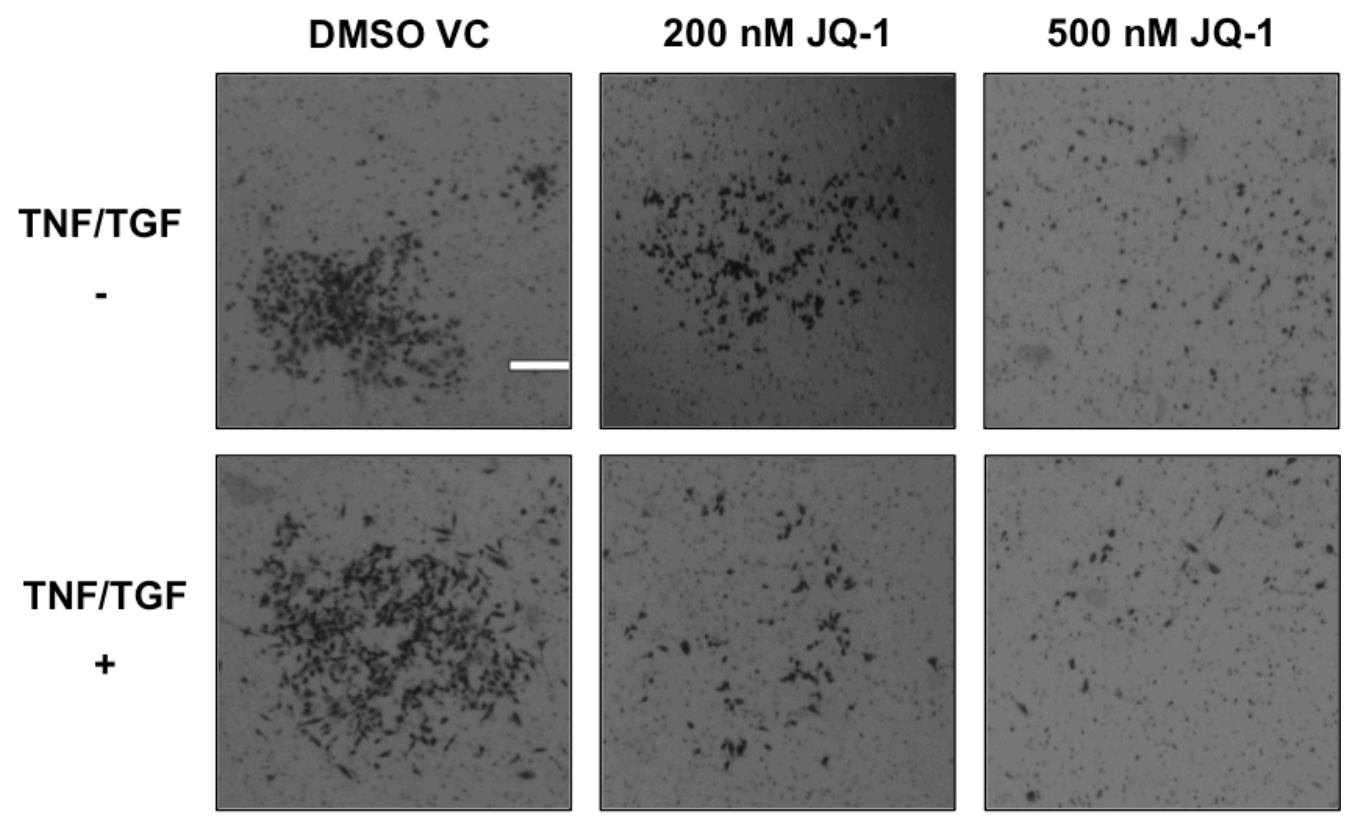

c.

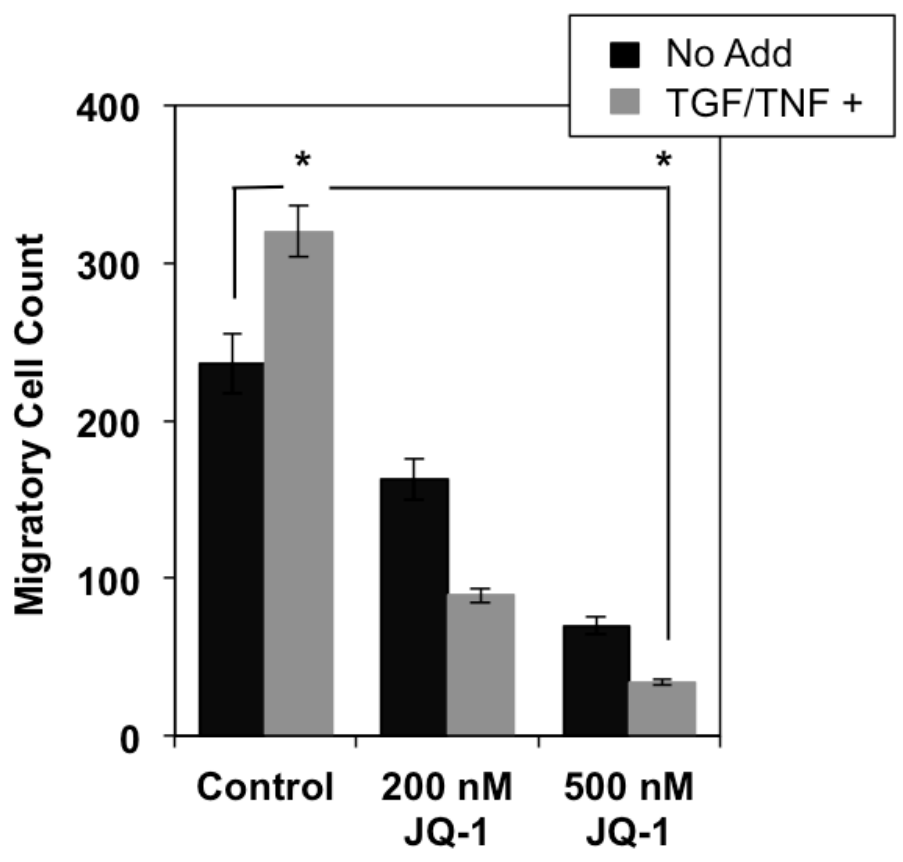


D.

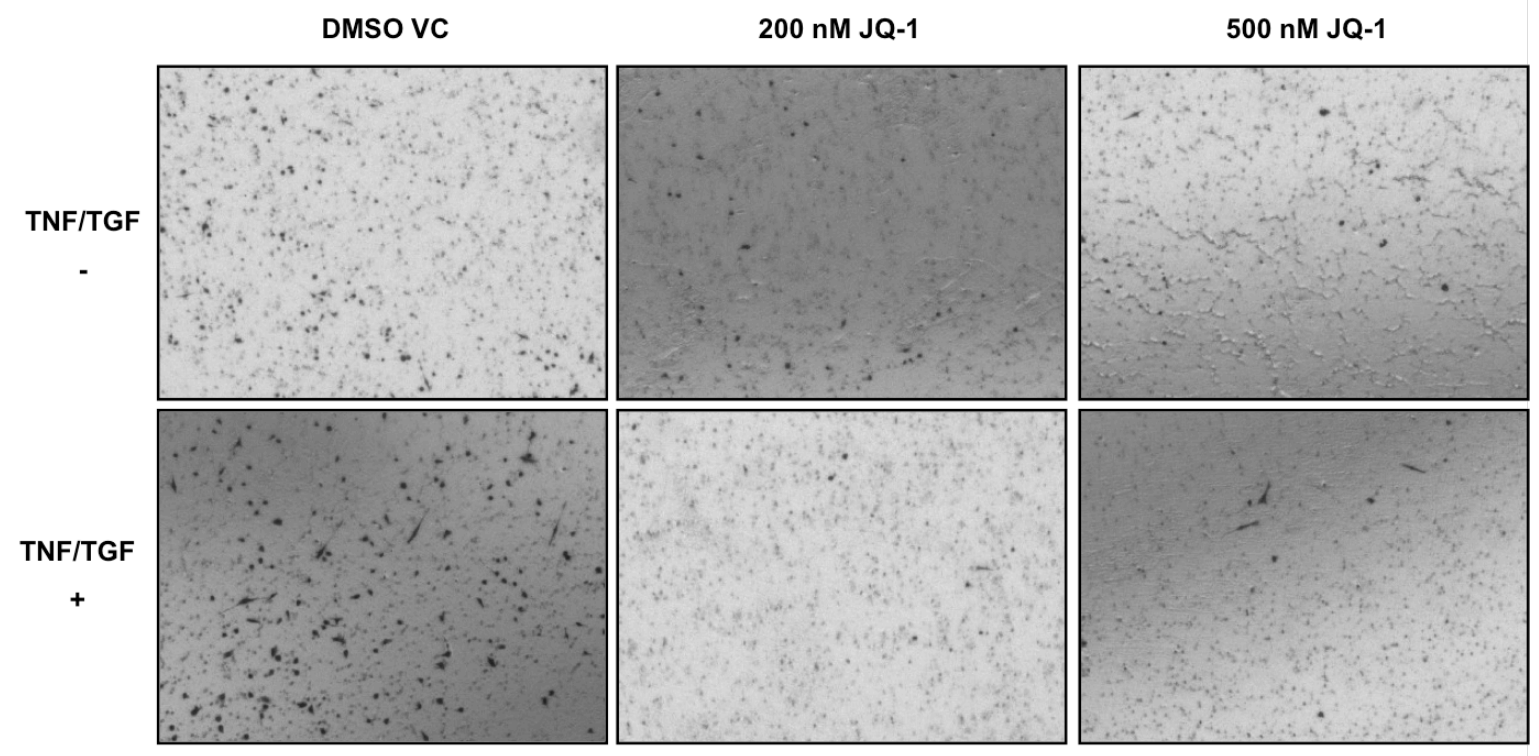

E.

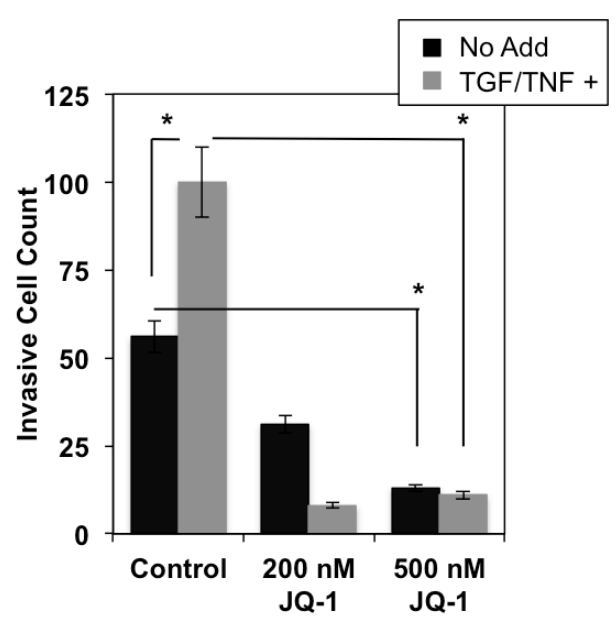


Figure 14. JQ1 impedes migration and invasion in tumor sphere NSCLC cultures. (A) qRT-PCR for matrix metalloproteinases (MMP1, MMP2, MMP3, and MMP10) in tumor sphere A549 treated with or without JQ1 and TGF $\beta$ + TNF; RNA levels were normalized to GAPDH. (B) Representative images of migratory cells in a transwell migration assay; cells were dissociated from tumor sphere A549 treated with or without JQ1 and TGF $\beta+$ TNF and plated in the transwells for 18 hours. (C) Cell counts of migratory cells in (B). (D) Representative images of invasive cells in a transwell invasion assay; cells were dissociated from tumor sphere A549 treated with or without JQ1 and TGF $\beta+$ TNF and plated in the transwells for 24 hours. (E) Cell counts of invasive cells in (D). 


\section{DISCUSSION}

Testing the effects of JQ1 in a tumor sphere model of NSCLC EMT has provided preliminary yet meaningful results. JQ1 inhibits expression of the master-switch transcription factors, which have all been shown to be associated with poor prognosis, disease progression, and metastasis in numerous solid malignancies including NSCLC (Bae et al., 2013; Kang and Massagué, 2004; Kaufhold and Bonavida, 2014; Kurrey et al., 2009; Mao et al., 2013). Previous studies have shown the importance of these transcription factors in facilitating EMT to produce invasive and migratory mesenchymal cells. It is unclear if the dramatic effects of JQ1 on the master-switch transcription factors seen in 3D cultures would also occur in an in vivo model of NSCLC.

While Fibronectin protein levels were decreased by the addition of JQ1, Vimentin and $\mathrm{N}$-cadherin protein levels remained unchanged following 96 hours of drug treatment. This was unexpected considering JQ1-treated cells failed to upregulate the master-switch transcription factors in response to cytokine stimulation. However, expression of the genes regulation Vimentin, Fibronectin, and $\mathrm{N}$-cadherin (VIM, FN1, and $C D H 2$, respectively) are not unilaterally regulated by the master-switch transcription factors (Lamouille et al., 2014). TGF $\beta$ stimulates expression of VIM through the AP-1 transcription factors FOS and Jun; and TGF $\beta$-induced $\mathrm{N}$-cadherin can occur via direct binding of SMAD4 to the CDH2 gene promoter (Kang et al., 2014; Wu et al., 2007). Further experimentation would be required to probe the effects of JQ1 on the activity of a wider array of EMT-associated transcription factors and their target genes. 
It is significant to note that repression of E-cadherin has also been shown to potentiate metastatic progression (von Burstin et al., 2009). AP-2 is the transcription factor associated with upregulation of $C D H 1$ gene. Interestingly, Myc has been shown to stimulate transcription of $C D H 1$ by AP-2. It follows that loss of Myc due to BET inhibition would result in repression of $\mathrm{CDH} 1$ despite the absence of the master-switch transcription factors that normally repress $\mathrm{CDH} 1$ during EMT (Batsché et al., 1998). E-cadherin is important in maintaining cellcell adherens junctions to maintain epithelial tissue organization. In the context of BET inhibition, it is unclear how the effect of decreased $C D H 1$ expression would manifest in vivo.

In the previous chapter, BET inhibition was shown to increase the migratory phenotype of PDAC cells in a scratch assay - this could potentially be due, in part, to the loss of E-cadherin in these cells. However, preliminary data presented in Figure 14 demonstrate that JQ1 has the notable effect of reducing NSCLC migration and invasion in transwell assays. Impairment of these phenotypes may be linked to the downregulation of MMP genes following JQ1 treatment. These results imply that JQ1 would impede CIC dissemination, and warrants testing in an in vivo model of NSCLC metastatic tumor formation.

\section{FUTURE DIRECTIONS}

\section{Evaluating the ability of BET inhibition to block metastasis in a NSCLC model of metastatic disease.}

Our lab has previously shown that subcutaneous (SC) injection of cells from cytokine-treated tumor sphere NSCLC cultures are more likely to 
metastasize to the lung than cells from cytokine-naïve tumor spheres. Following injection, cytokine-naïve NSCLC cells form tumors at the site of injection (Kumar et al., 2013; Wamsley et al., 2015). Considering the ability of JQ1 to block invasion and migration of cytokine treated A549 cells, it would be reasonable to assume that BET inhibition would also block the ability of cytokine-treated A549 cells to metastasize in vivo.

To perform this experiment, A549 cultures will be taken through tumor sphere culture and will either be left untreated or be treated with TNF and TGF for 96 hours. On the day of injection, tumor spheres will be trypsinized, resuspended in DMEM with $0.5 \%$ FBS before being counted and diluted in the appropriate cell concentration for injection. Cells will be subcutaneously injected into ten nude mice per cytokine condition (20 mice total). Treatment with the IBET151 would begin 24 hours following SC injections. Five of the mice injected with cytokine-naïve cells will receive $25 \mathrm{mg} / \mathrm{kg}$ of I-BET151 in a $0.5 \%$ hydroxypropyl solution delivered daily by oral gavage. The remaining five mice injected with cytokine-naïve cells will receive the vehicle control daily. Mice injected with the cytokine-treated cells will be similarly treated - five will be treated with I-BET151 and five will only receive the vehicle control.

Mice will be sacrificed after 4 weeks of treatment and the primary SC tumors will be removed and weighed. Additionally, the lungs will be removed and formalin fixed in order to count the number of surface lung metastases. Total tumor burden in the formalin fixed lung tissue will be quantified by qRT-PCR for human genomic material. Genomic DNA will be extracted and assayed for the 
abundance of human endogenous retrovirus-3 (ERV3) to confirm that metastatic lesions in the lungs of the animals arose from the injected human NSCLC cells.

Under these experimental conditions, it would be expected that the cytokine-naïve cells would produce larger tumors at the site of SC injection than the cytokine-treated cells. Conversely, the number of lung metastases would be greater in animals that received the cytokine-treated cells compared to animals injected with cytokine-naïve cells. Based on the ability of BET inhibition to block proliferation in NSCLC, we would expect the I-BET151 drug to reduce the size of SC tumors. In line with the transwell invasion and migration data shown in Figure 14, we hypothesize that animals injected with cytokine-treated cells would present with fewer lung tumors and reduced tumor burden if treated with IBET151. If out hypothesis is correct, these experiments would show that BET inhibition has the ability to block metastasis of NSCLC in vivo. 
CHAPTER 5: GENERAL DISCUSSION \& FUTURE DIRECTIONS 


\section{DISCUSSION}

Overcoming oncogene addition in response to targeted therapy requires transcriptional reprogramming, and often occurs through the upregulation of dedifferentiation and stem-like pathways. In our PDAC study, inhibition of the constitutively active oncogenic KRAS pathway with trametinib results in a population of cells that upregulates transcription of EMT and $\mathrm{CIC}$ genes. The addition of the BET inhibitor reduces trametinib-induced gene expression changes. Similarly, cytokine stimulation of NSCLC induces gene expression of the EMT transcriptional program. Again, the addition of a BET inhibitor reduces expression of these cytokine-induced genes. Consistent with the understanding that BET proteins recruit transcriptional machinery to active chromatin, our results suggest that $B E T$ inhibition disrupts the alterations in gene expression necessary for the acquisition of drug resistance that can occur in response to EMT.

Numerous studies have shown that BET inhibition downregulates expression of stem cell transcription factor and proto-oncogene $M y c$, but the influence of BET inhibition on EMT and cancer-initiating cells has not been fully explored in PDAC or NSCLC cancers. Moreover, the pleotropic effects of BET inhibitors are sure to include additional factors besides Myc. One recent study showed the ability of JQ1 to impede colony growth of PDAC cells in 3D collagen, a process that requires stem cell properties and the ability to differentiate.

Furthermore, JQ1 exhibited similar effects on restraining colony growth when the PDAC cells were made drug-resistant to the chemotherapy 5'fluorouracil (Sahai 
et al., 2014). Other groups have seen attenuation of CIC phenotypes following BET inhibition in other cancer types. For example, in vitro and in vivo, JQ1 hinders neuroblastoma progression and promotes neural differentiation of stemlike cancer cells (Lee et al., 2015). In Myc-driven medulloblastoma model, JQ1 attenuates stem cell signaling in vitro and impedes tumor growth in an in vivo xenograft model (Venkataraman et al., 2014).

Importantly, the inhibition of stemness via BET inhibition is not unique to cancer-stem cells, and has also been studied in embryonic and umbilical stem cells. ChIP-seq analysis in embryonic stem cells (ESC) demonstrated that BRD4 and $\mathrm{H} 4$ acetylation co-occupy sites in the genome that are upstream of pluripotency genes such as OCT4 and Nanog. During differentiation, there was a concomitant decrease in BRD4 protein levels and global histone $\mathrm{H} 4$ acetylation. Furthermore, JQ1 disrupted the interaction of BRD4 with histone $\mathrm{H} 4$, and resulted in enhanced differentiation (Gonzales-Cope et al., 2016). In umbilical cord-derived mesenchymal stem cells (MSCs), JQ1 down-regulates genes involved in self-renewal, cell cycle, DNA replication, and mitosis; this likely reduces the regenerative potential of MSCs (Alghamdi et al., 2016). Altogether, these studies affirm the theory that BET inhibitors have the capacity to disrupt super-enhancer functions associated with pluripotency. In doing so, these drugs may reduce the population of $\mathrm{CIC}$ cells responsible for relapse in refractory cancers.

Exploration of BET inhibitor therapies in PDAC and NSCLC may provide translational insight for other malignancies. TCGA data shows that BRD4 is 
overexpressed in roughly $30 \%$ of ovarian cancers, another tumor type that has low survivability. Thus far, only two studies have tested BET inhibitors in this cancer type; one study found that JQ1 blocks proliferation via FoxM1 (Zhang et al., 2016). FoxM1 is also important for regulation of EMT master-switch transcription factors and its overexpression is associated with pluripotency and metastasis in multiple cancer types (Meng et al., 2015; Yang et al., 2013). Preliminary studies in our lab have also confirmed the anti-proliferative effect of BET inhibition in ovarian cancer cell line SKOV3. We are currently performing experiments to understand the effects of BET inhibition on EMT and metastasis in ovarian cancer. The anti-proliferative effect of BET inhibition in cancer has been widely studied and clinical trials are underway to test the efficacy of these drugs in meaningfully quelling cancer progression in patients.

\section{FUTURE DIRECTIONS}

\section{Confirm the effect of BET inhibition on the disruption of super-enhancers in} KRAS-mutant PDAC and NSCLC.

Central to the hypothesis that BET inhibition has anti-tumor effects in PDAC and NSCLC is the idea that BET proteins facilitate the interaction of transcription factors, P-TEFb, Mediator, and RNA Pol II at sites of activated chromatin. As shown in Supplementary Figure S6A, the addition of I-BET151 to 366 tumor spheres reduced the prevalence of histone marks associated with BRD4 binding sites. We observed an overall loss of histone H3K14Ac and H4$\mathrm{K} 5, \mathrm{~K} 8, \mathrm{~K} 12$, and $\mathrm{K} 16$ acetylation using modification-specific antibodies in immunoblot assays. This observation could be explained by concomitant 
displacement of BRD4 and HATs, and/or increased HDAC activity following dissociation of BRD4 from acetylated lysines (Fig. S6B).

It would be interesting to determine whether changes in the localization of BET proteins occur during periods of phenotypic switching throughout the progression of cancer. This question would address whether: (1) BET proteins relocate to different regions of the genome during EMT or during the acquisition of drug resistance; (2) whether BET inhibition blocks transcriptional changes and results in epigenetic changes; (3) whether such mechanisms would occur at gene loci associated with stemness, cancer, and apoptosis. To address these questions, ChIP-Seq for BRD4, H3K14Ac, H4K5Ac, H4K8Ac, H4K12Ac, and H4K16Ac would be performed. To determine whether BET inhibition displaces BRD4 from binding within enhancer regions of the genome, ChIP-Seq would also be performed for enhancer markers H3K4me1, H3K4me2, H3K27ac. Additionally, it would be interesting to perform RNA-Seq to determine whether BET inhibition changes gene expression associated with alterations in histone acetylation and BRD4 localization.

Analysis of this data would require bioinformatics techniques to overlay BRD4 binding with the acetylation marks and enhancer-associated marks along the genome. Additionally, we would look for gene expression changes for genes in relative proximity (enhancer marks within $1 \mathrm{Mb}$ of a gene's cis-region) to BRD4-associated enhancers and promoters. Gene ontology analysis would show what cellular processes are activated during drug resistance and EMT, and 
what cellular processes are disrupted by BET inhibition during these reprogramming events. 


\section{SUPPLEMENTARY FIGURES}


A.

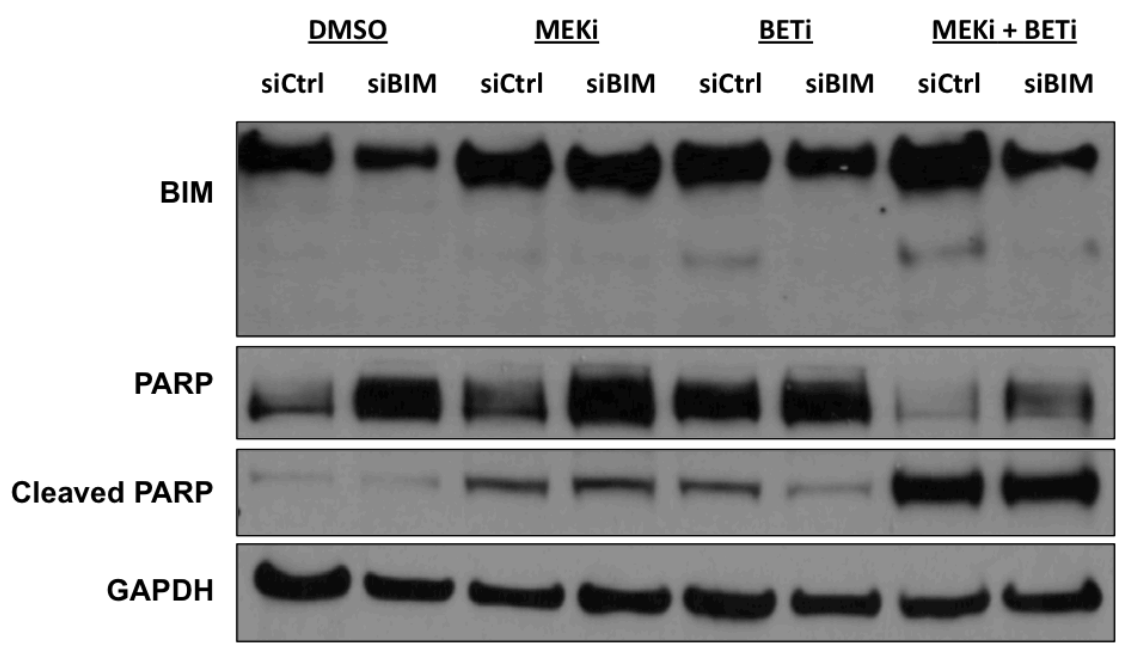

B.

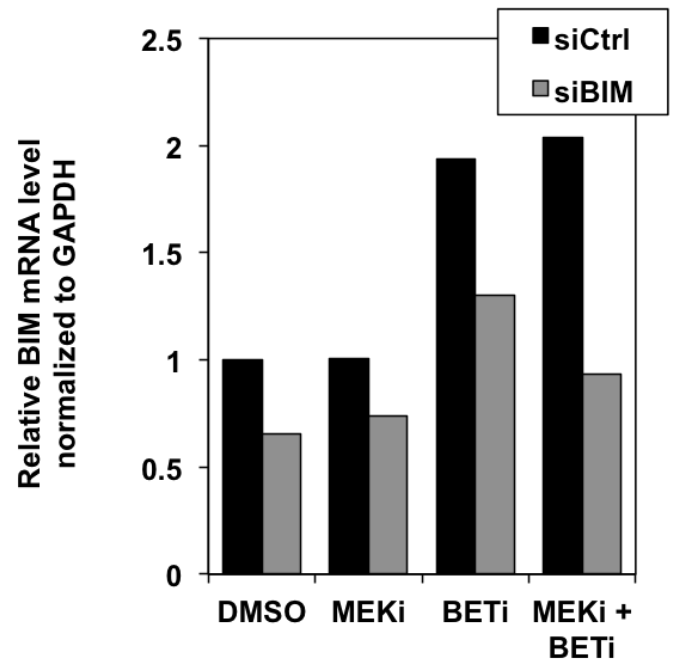

c.

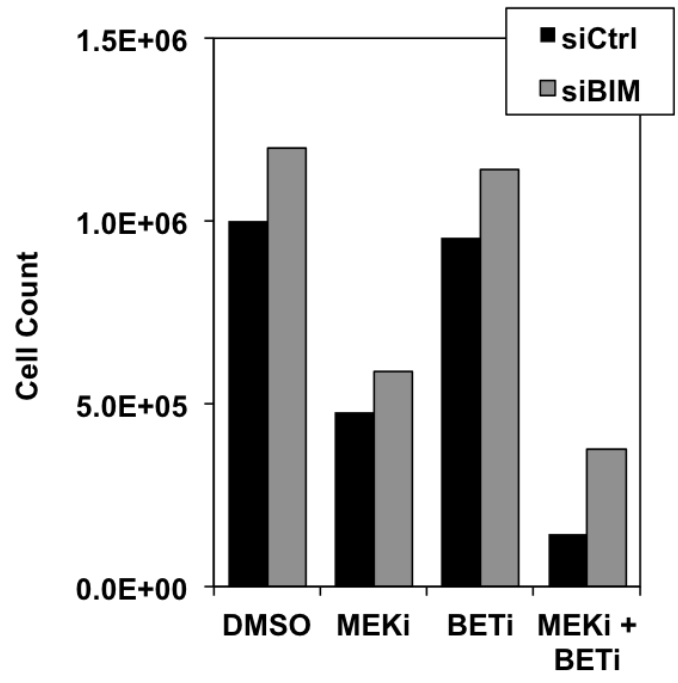


Figure S1. Knockdown of BIM using siRNA partially rescues the apoptotic phenotype. (A) Immunoblot showing protein levels of BIM in 608 cells transfected with siRNA against BIM. Cleaved PARP is shown to indicate commitment to apoptosis, and GAPDH is used as a loading control. (B) qRTPCR to measure knockdown of BIM mRNA expression in 608 cells transfected with siRNA against BIM. (C) Cell counts from a tumor sphere replating assay of 608 cells transfected with siRNA, taken through tumor sphere culture and treated with the indicated inhibitors for 72 hours. 


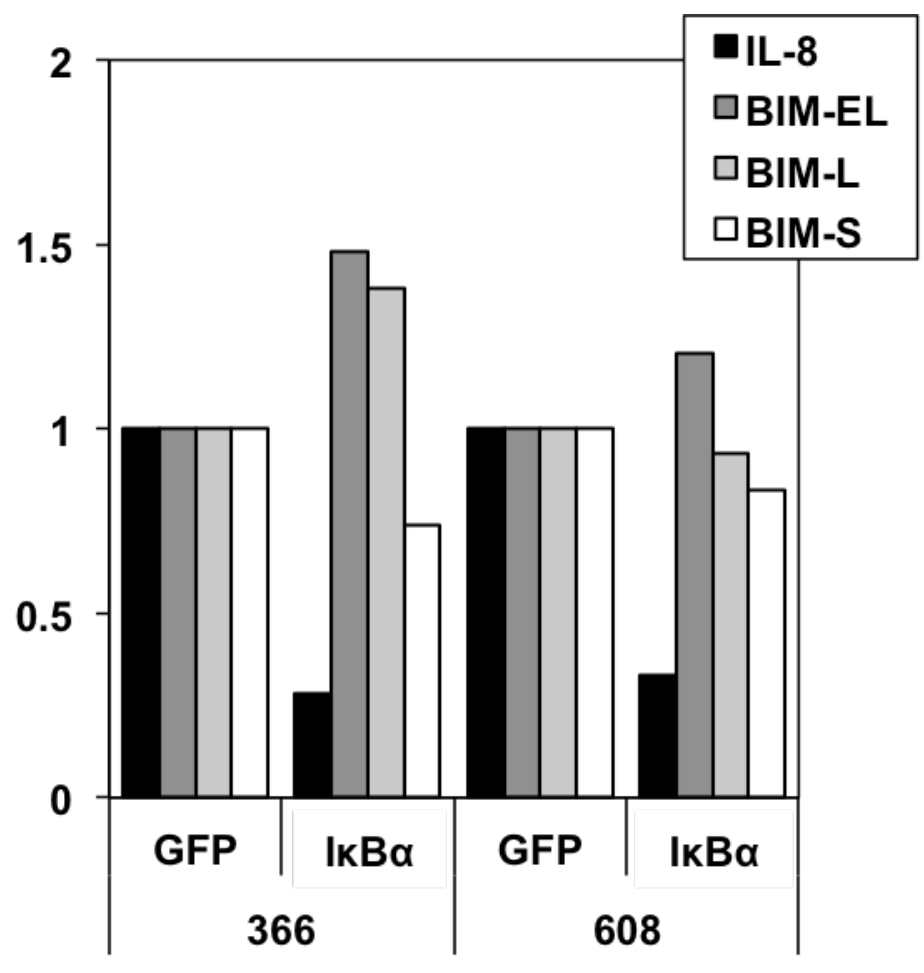


Figure S2. The super repressor of NF-kB fails to induce expression of BIM in 366 and 608 PDAC cells. qRT-PCR for expression BIM mRNA splice forms in 366 and 608 cells infected with adenovirus overexpressing $1 \kappa B \alpha$. Expression was normalized to GAPDH. The NF-kB target gene IL-8 was used as a positive control for IKB $\alpha$ activity. 


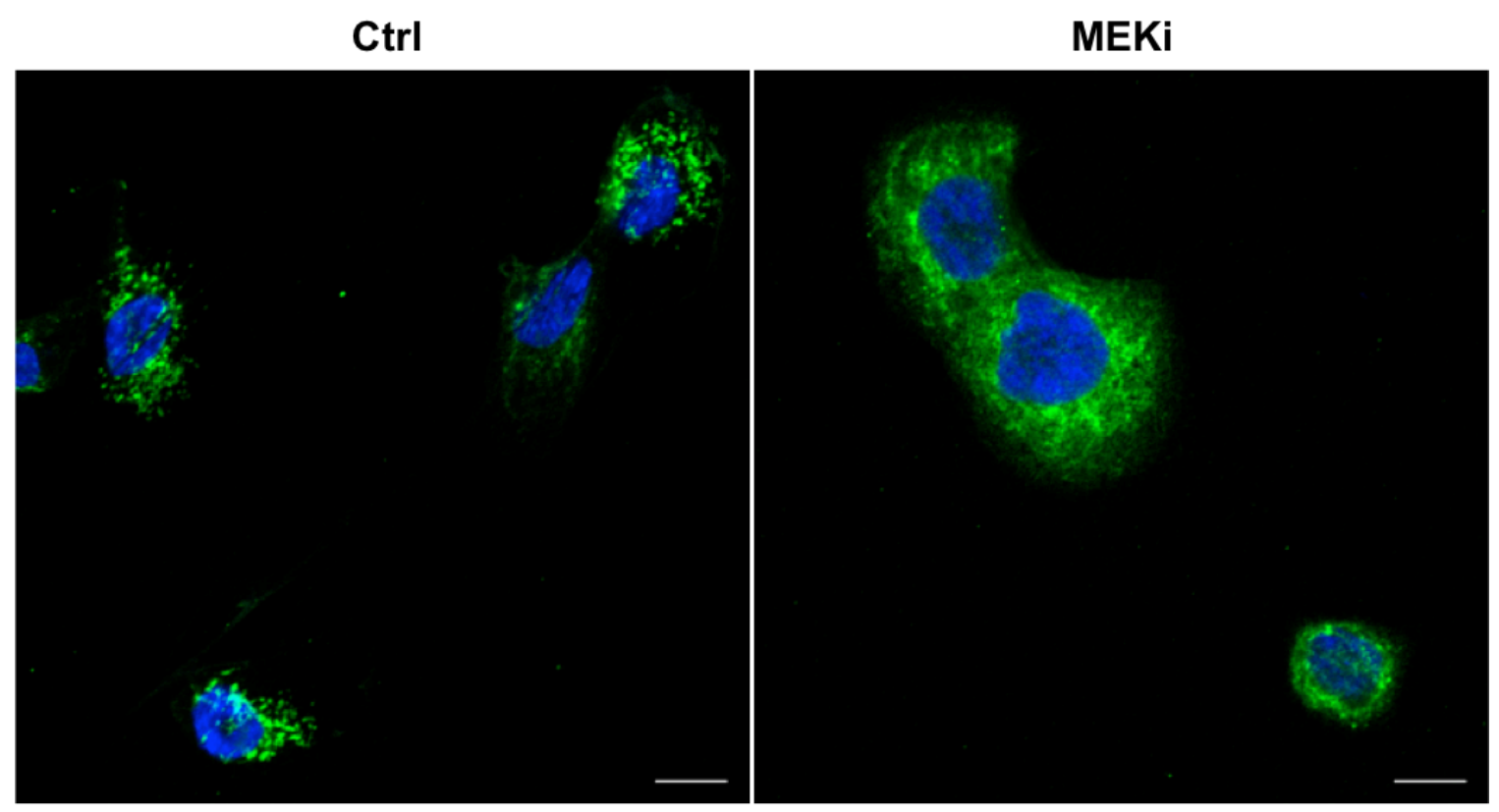

\section{BETi}

$M E K i+B E T i$

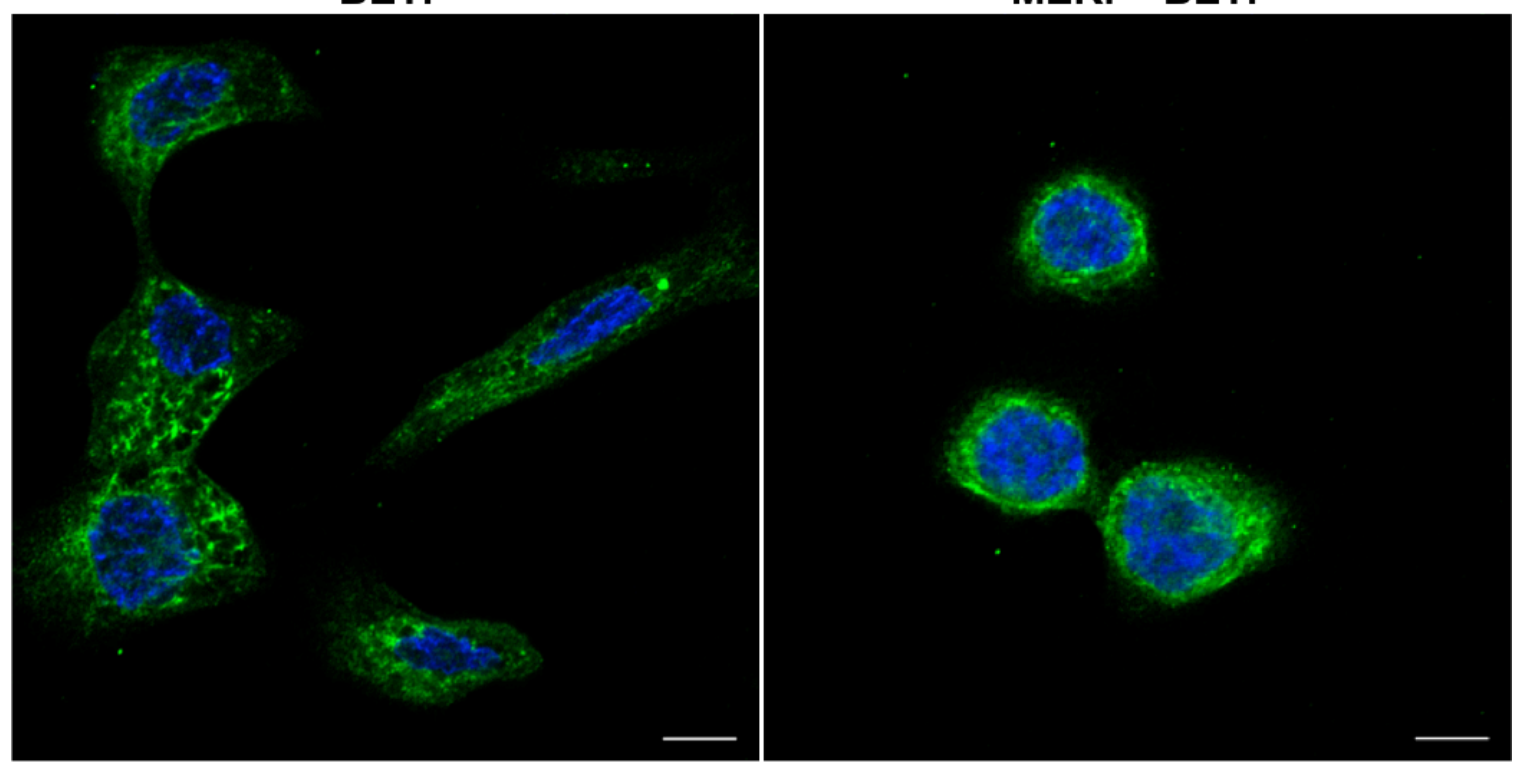


Figure S3. Trametinib and I-BET151 increase mitochondrial network connectivity in 2D PDAC cultures. 366 cells were seeded on cover slips before being treated with the indicated inhibitors for 48 hours. Nuclei were stained with DAPI, and the outer mitochondrial membrane was stained with antiTOM20 and a secondary antibody conjugated to a 488 fluorophore. Staining and imaging performed by Sarbjeet Nagdas in the laboratory of Dr. David Kashatus (University of Virginia). 


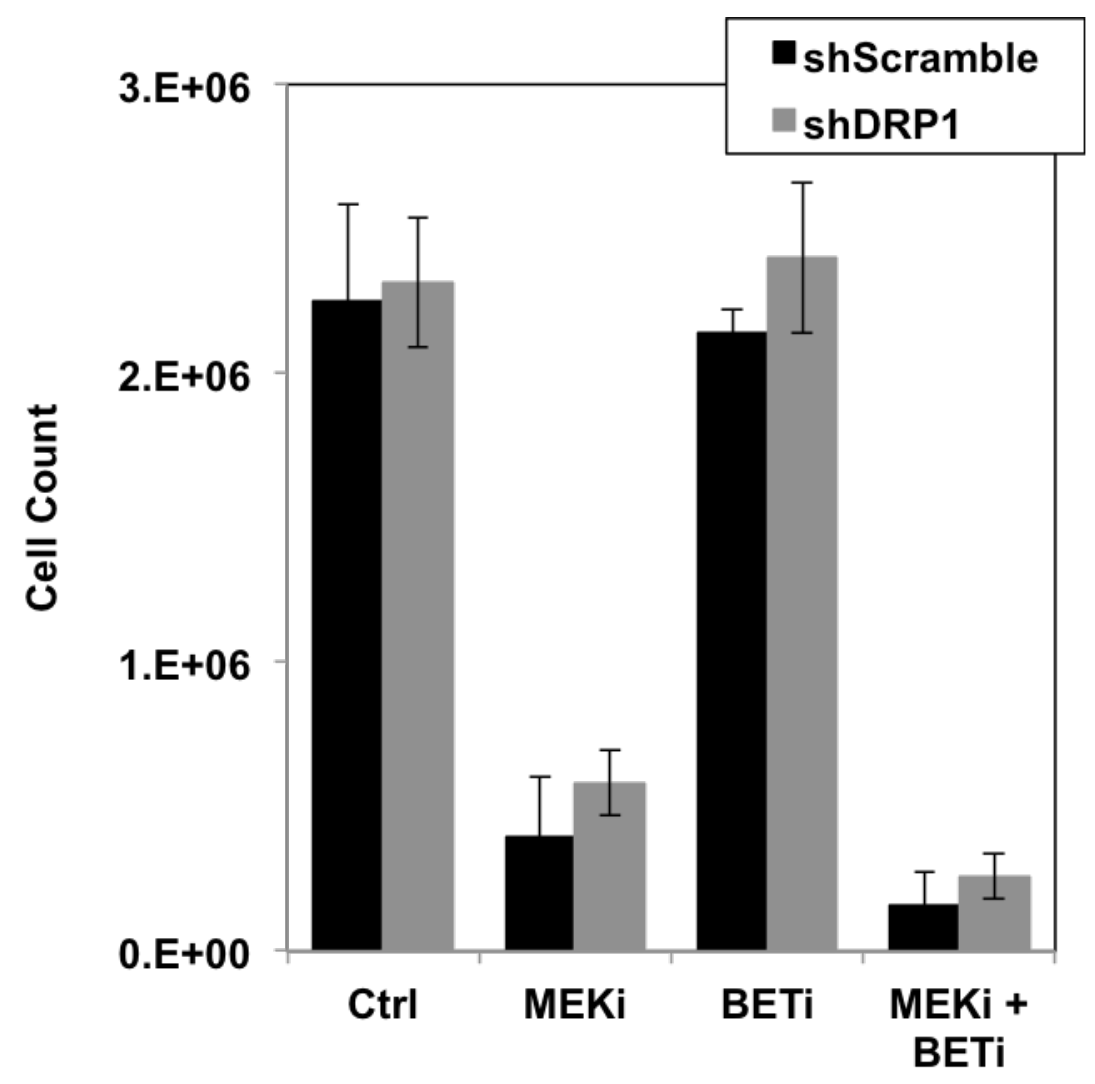

Inhibitor Treatment 
Figure S4. I-BET151 fails to induce apoptosis in DRP1 knockdown PDAC cells. Tumor spheres cultures were made from mPanc96 cells stably expressing shRNA against DRP1 or a scramble control shRNA. Tumor spheres were treated with inhibitors as indicated for 96 hours before cells were dissociated, replated for 24 hours, and counted. 


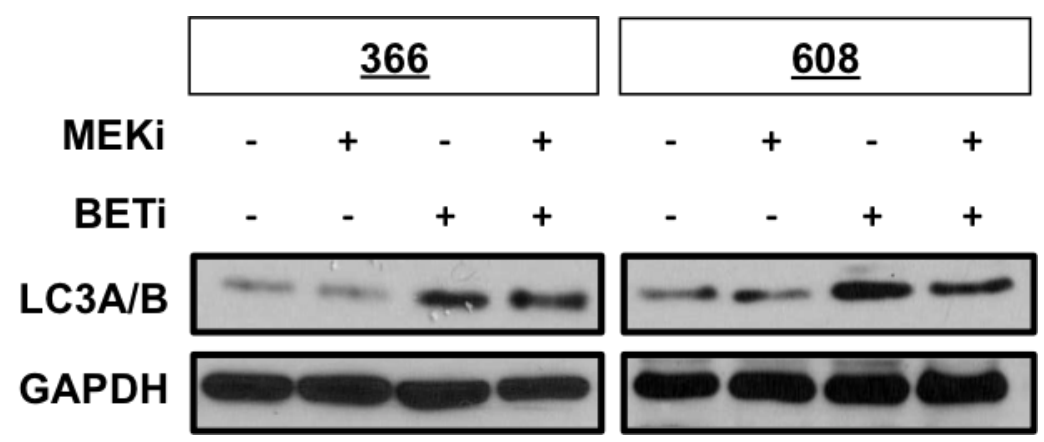


Figure S5. I-BET151 induces autophagy marker LC3A/B in 3D PDAC

cultures. Immunoblots probed for autophagy marker LC3A/B in 366 and 608 tumor spheres treated with the indicated inhibitors for 24 hours. 
A.

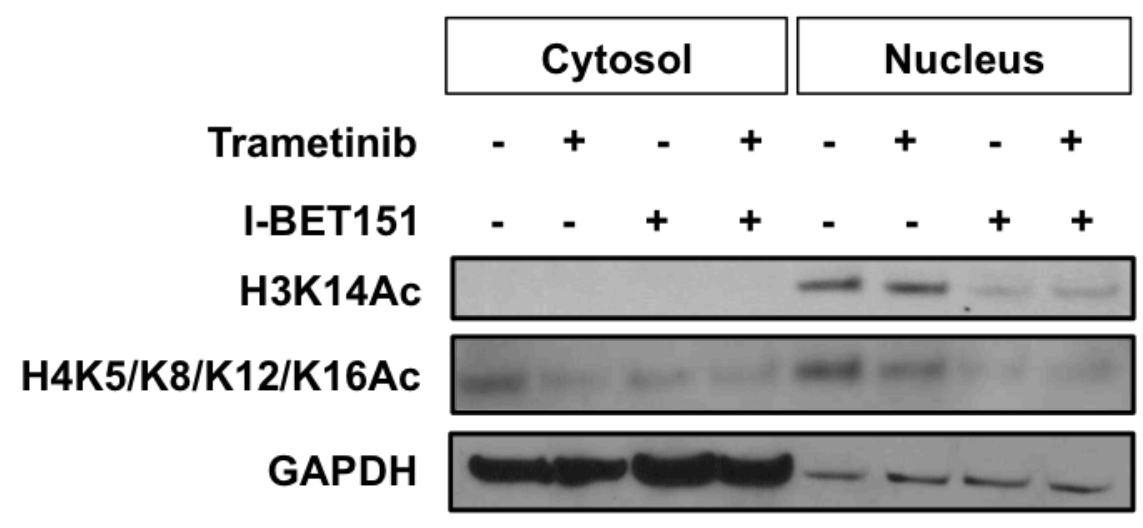

B.

Hypothesis

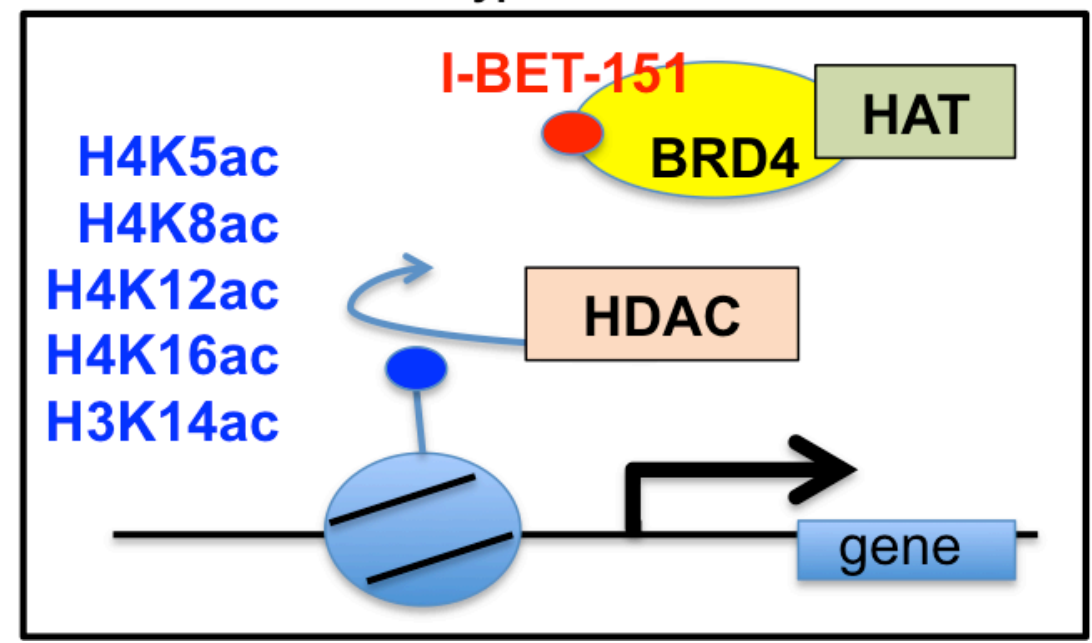


Figure S6. I-BET151 alters histone acetylation profiles in 366 cells. (A) Immunoblot for acetylated histones in cytosolic and nuclear fractions of 366 tumor spheres treated with the indicated inhibitors for 48 hours. (B) A model illustrating possible mechanisms by which BRD4 dissociation from acetylated histones results in a loss of chromatin-associated HATS and/or increases accessibility to HDACs to reduce histone acetylation. 


\section{REFERENCES}

Aghdassi, A., Sendler, M., Guenther, A., Mayerle, J., Behn, C.-O., Heidecke, C.D., Friess, H., Büchler, M., Evert, M., Lerch, M.M., et al. (2012). Recruitment of histone deacetylases HDAC1 and HDAC2 by the transcriptional repressor ZEB1 downregulates E-cadherin expression in pancreatic cancer. Gut 61, 439-448.

Aisner, D.L., and Marshall, C.B. (2012). Molecular pathology of non-small cell lung cancer: a practical guide. Am. J. Clin. Pathol. 138, 332-346.

Akhtar-Zaidi, B., Cowper-Sal-lari, R., Corradin, O., Saiakhova, A., Bartels, C.F., Balasubramanian, D., Myeroff, L., Lutterbaugh, J., Jarrar, A., Kalady, M.F., et al. (2012). Epigenomic enhancer profiling defines a signature of colon cancer. Science 336, 736-739.

Alghamdi, S., Khan, I., Beeravolu, N., McKee, C., Thibodeau, B., Wilson, G., and Chaudhry, G.R. (2016). BET protein inhibitor JQ1 inhibits growth and modulates WNT signaling in mesenchymal stem cells. Stem Cell Res. Ther. 7, 22.

Allan, L.A., Morrice, N., Brady, S., Magee, G., Pathak, S., and Clarke, P.R. (2003). Inhibition of caspase- 9 through phosphorylation at Thr 125 by ERK MAPK. Nat. Cell Biol. 5, 647-654.

Aprea, M.C., Bosi, A., Manara, M., Mazzocchi, B., Pompini, A., Sormani, F., Lunghini, L., and Sciarra, G. (2016). Assessment of exposure to pesticides during mixing/loading and spraying of tomatoes in the open field. J. Occup. Environ. Hyg. 1-37.

Arlt, A., Müerköster, S.S., and Schäfer, H. (2013). Targeting apoptosis pathways in pancreatic cancer. Cancer Lett. 332, 346-358.

Arumugam, T., Ramachandran, V., Fournier, K.F., Wang, H., Marquis, L., Abbruzzese, J.L., Gallick, G.E., Logsdon, C.D., McConkey, D.J., and Choi, W. (2009). Epithelial to mesenchymal transition contributes to drug resistance in pancreatic cancer. Cancer Res. 69, 5820-5828.

Bach, P.B., Mirkin, J.N., Oliver, T.K., Azzoli, C.G., Berry, D.A., Brawley, O.W., Byers, T., Colditz, G.A., Gould, M.K., Jett, J.R., et al. (2012). Benefits and harms of CT screening for lung cancer: a systematic review. JAMA 307, 2418-2429.

Bachmann, P.S., Piazza, R.G., Janes, M.E., Wong, N.C., Davies, C., Mogavero, A., Bhadri, V.A., Szymanska, B., Geninson, G., Magistroni, V., et al. (2010). Epigenetic silencing of BIM in glucocorticoid poor-responsive pediatric acute lymphoblastic leukemia, and its reversal by histone deacetylase inhibition. Blood 116, 3013-3022.

Bae, G.-Y., Choi, S.-J., Lee, J.-S., Jo, J., Lee, J., Kim, J., and Cha, H.-J. (2013). Loss of E-cadherin activates EGFR-MEK/ERK signaling, which promotes 
invasion via the ZEB1/MMP2 axis in non-small cell lung cancer. Oncotarget 4 , 2512-2522.

Barski, A., Cuddapah, S., Cui, K., Roh, T.-Y., Schones, D.E., Wang, Z., Wei, G., Chepelev, I., and Zhao, K. (2007). High-resolution profiling of histone methylations in the human genome. Cell 129, 823-837.

Batsché, E., Muchardt, C., Behrens, J., Hurst, H.C., and Crémisi, C. (1998). RB and C-Myc activate expression of the E-cadherin gene in epithelial cells through interaction with transcription factor AP-2. Mol. Cell. Biol. 18, 3647-3658.

Bellaye, P.-S., Burgy, O., Causse, S., Garrido, C., and Bonniaud, P. (2014). Heat shock proteins in fibrosis and wound healing: good or evil? Pharmacol. Ther. 143, 119-132.

Bhattacharya, S., Socinski, M.A., and Burns, T.F. (2015). KRAS mutant lung cancer: progress thus far on an elusive therapeutic target. Clin. Transl. Med. 4, 35 .

Blanco, F.F., Jimbo, M., Wulfkuhle, J., Gallagher, I., Deng, J., Enyenihi, L., Meisner-Kober, N., Londin, E., Rigoutsos, I., Sawicki, J.A., et al. (2015). The mRNA-binding protein HuR promotes hypoxia-induced chemoresistance through posttranscriptional regulation of the proto-oncogene PIM1 in pancreatic cancer cells. Oncogene.

Blick, T., Hugo, H., Widodo, E., Waltham, M., Pinto, C., Mani, S.A., Weinberg, R.A., Neve, R.M., Lenburg, M.E., and Thompson, E.W. (2010). Epithelial mesenchymal transition traits in human breast cancer cell lines parallel the CD44(hi/)CD24 (lo/-) stem cell phenotype in human breast cancer. J. Mammary Gland Biol. Neoplasia 15, 235-252.

Blum, R., and Kloog, Y. (2014). Metabolism addiction in pancreatic cancer. Cell Death Dis. 5, e1065.

Bridgeman, V.L., Wan, E., Foo, S., Nathan, M.R., Welti, J.C., Frentzas, S., Vermeulen, P.B., Preece, N., Springer, C.J., Powles, T., et al. (2016). Preclinical Evidence That Trametinib Enhances the Response to Antiangiogenic Tyrosine Kinase Inhibitors in Renal Cell Carcinoma. Mol. Cancer Ther. 15, 172-183.

Brouchet, L., Valmary, S., Dahan, M., Didier, A., Galateau-Salle, F., Brousset, P., and Degano, B. (2005). Detection of oncogenic virus genomes and gene products in lung carcinoma. Br. J. Cancer 92, 743-746.

Von Burstin, J., Eser, S., Paul, M.C., Seidler, B., Brandl, M., Messer, M., von Werder, A., Schmidt, A., Mages, J., Pagel, P., et al. (2009). E-cadherin regulates metastasis of pancreatic cancer in vivo and is suppressed by a SNAIL/HDAC1/HDAC2 repressor complex. Gastroenterology 137, 361-371, 371.e1-e5. 
Chamorro-Jorganes, A., Lee, M.Y., Araldi, E., Landskroner-Eiger, S., FernándezFuertes, M., Sahraei, M., Quiles Del Rey, M., van Solingen, C., Yu, J., Fernández-Hernando, C., et al. (2016). VEGF-Induced Expression of miR-17-92 Cluster in Endothelial Cells Is Mediated by ERK/ELK1 Activation and Regulates Angiogenesis. Circ. Res. 118, 38-47.

Chand, S., O'Hayer, K., Blanco, F.F., Winter, J.M., and Brody, J.R. (2016). The Landscape of Pancreatic Cancer Therapeutic Resistance Mechanisms. Int. J. Biol. Sci. 12, 273-282.

Chang, D.K., Grimmond, S.M., and Biankin, A.V. (2014). Pancreatic cancer genomics. Curr. Opin. Genet. Dev. 24, 74-81.

Chang, Q., Jurisica, I., Do, T., and Hedley, D.W. (2011). Hypoxia predicts aggressive growth and spontaneous metastasis formation from orthotopically grown primary xenografts of human pancreatic cancer. Cancer Res. 71, 31103120.

Chang, S.Y., Keeney, M., Law, M., Donovan, J., Aubry, M.-C., and Garcia, J. (2015). Detection of human papillomavirus in non-small cell carcinoma of the lung. Hum. Pathol. 46, 1592-1597.

Chen, W., Li, Z., Bai, L., and Lin, Y. (2011). NF-kappaB in lung cancer, a carcinogenesis mediator and a prevention and therapy target. Front. Biosci. Landmark Ed. 16, 1172-1185.

Chiorean, E.G., and Coveler, A.L. (2015). Pancreatic cancer: optimizing treatment options, new, and emerging targeted therapies. Drug Des. Devel. Ther. 9, 3529-3545.

Cieślik, M., Hoang, S.A., Baranova, N., Chodaparambil, S., Kumar, M., Allison, D.F., Xu, X., Wamsley, J.J., Gray, L., Jones, D.R., et al. (2013). Epigenetic coordination of signaling pathways during the epithelial-mesenchymal transition. Epigenetics Chromatin 6, 28.

Cohen, R., Neuzillet, C., Tijeras-Raballand, A., Faivre, S., de Gramont, A., and Raymond, E. (2015). Targeting cancer cell metabolism in pancreatic adenocarcinoma. Oncotarget 6, 16832-16847.

Correia, C., Lee, S.-H., Meng, X.W., Vincelette, N.D., Knorr, K.L.B., Ding, H., Nowakowski, G.S., Dai, H., and Kaufmann, S.H. (2015). Emerging understanding of $\mathrm{Bcl}-2$ biology: Implications for neoplastic progression and treatment. Biochim. Biophys. Acta BBA - Mol. Cell Res. 1853, 1658-1671.

Crews, C.M., Alessandrini, A., and Erikson, R.L. (1992). The primary structure of MEK, a protein kinase that phosphorylates the ERK gene product. Science 258, 478-480. 
Creyghton, M.P., Cheng, A.W., Welstead, G.G., Kooistra, T., Carey, B.W., Steine, E.J., Hanna, J., Lodato, M.A., Frampton, G.M., Sharp, P.A., et al. (2010). Histone H3K27ac separates active from poised enhancers and predicts developmental state. Proc. Natl. Acad. Sci. U. S. A. 107, 21931-21936.

Cufer, T., Ovcaricek, T., and O'Brien, M.E.R. (2013). Systemic therapy of advanced non-small cell lung cancer: major-developments of the last 5-years. Eur. J. Cancer Oxf. Engl. 1990 49, 1216-1225.

Dai, H., Ding, H., Meng, X.W., Lee, S.-H., Schneider, P.A., and Kaufmann, S.H. (2013). Contribution of Bcl-2 Phosphorylation to Bak Binding and Drug Resistance. Cancer Res. 73, 6998-7008.

D'Angelo, R.C., and Wicha, M.S. (2010). Stem cells in normal development and cancer. Prog. Mol. Biol. Transl. Sci. 95, 113-158.

David, S.P., Wang, A., Kapphahn, K., Hedlin, H., Desai, M., Henderson, M., Yang, L., Walsh, K.M., Schwartz, A.G., Wiencke, J.K., et al. (2016). Gene by Environment Investigation of Incident Lung Cancer Risk in African-Americans. EBioMedicine 4, 153-161.

Dawson, M.A., Prinjha, R.K., Dittmann, A., Giotopoulos, G., Bantscheff, M., Chan, W.-I., Robson, S.C., Chung, C., Hopf, C., Savitski, M.M., et al. (2011). Inhibition of BET recruitment to chromatin as an effective treatment for MLLfusion leukaemia. Nature 478, 529-533.

Delmore, J.E., Issa, G.C., Lemieux, M.E., Rahl, P.B., Shi, J., Jacobs, H.M., Kastritis, E., Gilpatrick, T., Paranal, R.M., Qi, J., et al. (2011). BET bromodomain inhibition as a therapeutic strategy to target c-Myc. Cell 146, 904-917.

Deng, W., Lee, J., Wang, H., Miller, J., Reik, A., Gregory, P.D., Dean, A., and Blobel, G.A. (2012). Controlling long-range genomic interactions at a native locus by targeted tethering of a looping factor. Cell 149, 1233-1244.

Deng, X., Ruvolo, P., Carr, B., and May, W.S. (2000). Survival function of ERK1/2 as IL-3-activated, staurosporine-resistant Bcl2 kinases. Proc. Natl. Acad. Sci. $97,1578-1583$.

Denis, G.V., McComb, M.E., Faller, D.V., Sinha, A., Romesser, P.B., and Costello, C.E. (2006). Identification of transcription complexes that contain the double bromodomain protein Brd2 and chromatin remodeling machines. J. Proteome Res. 5, 502-511.

Dey, A., Chitsaz, F., Abbasi, A., Misteli, T., and Ozato, K. (2003). The double bromodomain protein $\mathrm{Brd} 4$ binds to acetylated chromatin during interphase and mitosis. Proc. Natl. Acad. Sci. U. S. A. 100, 8758-8763. 
Dey, A., Nishiyama, A., Karpova, T., McNally, J., and Ozato, K. (2009). Brd4 marks select genes on mitotic chromatin and directs postmitotic transcription. Mol. Biol. Cell 20, 4899-4909.

Dimastromatteo, J., Houghton, J.L., Lewis, J.S., and Kelly, K.A. (2015).

Challenges of Pancreatic Cancer. Cancer J. Sudbury Mass 21, 188-193.

Długosz, A., and Janecka, A. (2016). ABC transporters in the development of multidrug resistance in cancer therapy. Curr. Pharm. Des.

Dobrila-Dintinjana, R., Vanis, N., Dintinjana, M., and Radić, M. (2012). Etiology and oncogenesis of pancreatic carcinoma. Coll. Antropol. 36, 1063-1067.

Donadelli, M., Dando, I., Zaniboni, T., Costanzo, C., Dalla Pozza, E., Scupoli, M.T., Scarpa, A., Zappavigna, S., Marra, M., Abbruzzese, A., et al. (2011). Gemcitabine/cannabinoid combination triggers autophagy in pancreatic cancer cells through a ROS-mediated mechanism. Cell Death Dis. 2, e152.

Dorris, E.R., Blackshields, G., Sommerville, G., Alhashemi, M., Dias, A., McEneaney, V., Smyth, P., O'Leary, J.J., and Sheils, O. (2016). Pluripotency markers are differentially induced by MEK inhibition in thyroid and melanoma BRAFV600E cell lines. Cancer Biol. Ther. 0.

Du, Z., Qin, R., Wei, C., Wang, M., Shi, C., Tian, R., and Peng, C. (2011).

Pancreatic cancer cells resistant to chemoradiotherapy rich in "stem-cell-like" tumor cells. Dig. Dis. Sci. 56, 741-750.

Eberlein, C.A., Stetson, D., Markovets, A.A., Al-Kadhimi, K.J., Lai, Z., Fisher, P.R., Meador, C.B., Spitzler, P., Ichihara, E., Ross, S.J., et al. (2015). Acquired Resistance to the Mutant-Selective EGFR Inhibitor AZD9291 Is Associated with Increased Dependence on RAS Signaling in Preclinical Models. Cancer Res. 75, 2489-2500.

Eser, S., Schnieke, A., Schneider, G., and Saur, D. (2014). Oncogenic KRAS signalling in pancreatic cancer. Br. J. Cancer 111, 817-822.

Eshoo, A. (2012). H.R.733 - 112th Congress (2011-2012): Recalcitrant Cancer Research Act of 2012.

Ewings, K.E., Wiggins, C.M., and Cook, S.J. (2007). Bim and the pro-survival Bcl-2 proteins: opposites attract, ERK repels. Cell Cycle Georget. Tex 6, 22362240.

Fang, B. (2016). RAS signaling and anti-RAS therapy: lessons learned from genetically engineered mouse models, human cancer cells, and patient-related studies. Acta Biochim. Biophys. Sin. 48, 27-38. 
Fang, W., Hong, S., Chen, N., He, X., Zhan, J., Qin, T., Zhou, T., Hu, Z., Ma, Y., Zhao, Y., et al. (2015). PD-L1 is remarkably over-expressed in EBV-associated pulmonary lymphoepithelioma-like carcinoma and related to poor disease-free survival. Oncotarget 6, 33019-33032.

Ferlay, J., Soerjomataram, I., Dikshit, R., Eser, S., Mathers, C., Rebelo, M., Parkin, D.M., Forman, D., and Bray, F. (2015). Cancer incidence and mortality worldwide: Sources, methods and major patterns in GLOBOCAN 2012. Int. J. Cancer 136, E359-E386.

Ferri, E., Petosa, C., and McKenna, C.E. (2016). Bromodomains: Structure, function and pharmacology of inhibition. Biochem. Pharmacol. 106, 1-18.

Filippakopoulos, P., Qi, J., Picaud, S., Shen, Y., Smith, W.B., Fedorov, O., Morse, E.M., Keates, T., Hickman, T.T., Felletar, I., et al. (2010). Selective inhibition of BET bromodomains. Nature 468, 1067-1073.

Fiskus, W., Sharma, S., Qi, J., Valenta, J.A., Schaub, L.J., Shah, B., Peth, K., Portier, B.P., Rodriguez, M., Devaraj, S.G.T., et al. (2014). Highly active combination of BRD4 antagonist and histone deacetylase inhibitor against human acute myelogenous leukemia cells. Mol. Cancer Ther. 13, 1142-1154.

Fraga, M.F., Ballestar, E., Villar-Garea, A., Boix-Chornet, M., Espada, J., Schotta, G., Bonaldi, T., Haydon, C., Ropero, S., Petrie, K., et al. (2005). Loss of acetylation at Lys 16 and trimethylation at Lys 20 of histone $\mathrm{H} 4$ is a common hallmark of human cancer. Nat. Genet. 37, 391-400.

Frisch, S.M., Schaller, M., and Cieply, B. (2013). Mechanisms that link the oncogenic epithelial-mesenchymal transition to suppression of anoikis. J. Cell Sci. 126, 21-29.

Fulda, S. (2009). Apoptosis pathways and their therapeutic exploitation in pancreatic cancer. J. Cell. Mol. Med. 13, 1221-1227.

Fuxe, J., Vincent, T., and Garcia de Herreros, A. (2010). Transcriptional crosstalk between TGF- $\beta$ and stem cell pathways in tumor cell invasion: role of EMT promoting Smad complexes. Cell Cycle Georget. Tex 9, 2363-2374.

Gallagher, S.J., Mijatov, B., Gunatilake, D., Gowrishankar, K., Tiffen, J., James, W., Jin, L., Pupo, G., Cullinane, C., McArthur, G.A., et al. (2014a). Control of NF$\mathrm{kB}$ activity in human melanoma by bromodomain and extra-terminal protein inhibitor I-BET151. Pigment Cell Melanoma Res. 27, 1126-1137.

Gallagher, S.J., Mijatov, B., Gunatilake, D., Tiffen, J.C., Gowrishankar, K., Jin, L., Pupo, G.M., Cullinane, C., Prinjha, R.K., Smithers, N., et al. (2014b). The epigenetic regulator I-BET151 induces BIM-dependent apoptosis and cell cycle arrest of human melanoma cells. J. Invest. Dermatol. 134, 2795-2805. 
Gamsjaeger, R., Webb, S.R., Lamonica, J.M., Billin, A., Blobel, G.A., and Mackay, J.P. (2011). Structural basis and specificity of acetylated transcription factor GATA1 recognition by BET family bromodomain protein Brd3. Mol. Cell. Biol. 31, 2632-2640.

Gao, X., Wu, X., Zhang, X., Hua, W., Zhang, Y., Maimaiti, Y., Gao, Z., and Zhang, Y. (2016). Inhibition of BRD4 suppresses tumor growth and enhances iodine uptake in thyroid cancer. Biochem. Biophys. Res. Commun. 469, 679685 .

Garcia, P.L., Miller, A.L., Kreitzburg, K.M., Council, L.N., Gamblin, T.L., Christein, J.D., Heslin, M.J., Arnoletti, J.P., Richardson, J.H., Chen, D., et al. (2016). The BET bromodomain inhibitor JQ1 suppresses growth of pancreatic ductal adenocarcinoma in patient-derived xenograft models. Oncogene 35, 833-845.

Gatzemeier, U., Pluzanska, A., Szczesna, A., Kaukel, E., Roubec, J., De Rosa, F., Milanowski, J., Karnicka-Mlodkowski, H., Pesek, M., Serwatowski, P., et al. (2007). Phase III study of erlotinib in combination with cisplatin and gemcitabine in advanced non-small-cell lung cancer: the Tarceva Lung Cancer Investigation Trial. J. Clin. Oncol. Off. J. Am. Soc. Clin. Oncol. 25, 1545-1552.

Gloss, B.S., and Samimi, G. (2014). Epigenetic biomarkers in epithelial ovarian cancer. Cancer Lett. 342, 257-263.

Gómez, V.E., Giovannetti, E., and Peters, G.J. (2015). Unraveling the complexity of autophagy: Potential therapeutic applications in Pancreatic Ductal Adenocarcinoma. Semin. Cancer Biol. 35, 11-19.

Gonzales-Cope, M., Sidoli, S., Bhanu, N.V., Won, K.-J., and Garcia, B.A. (2016). Histone $\mathrm{H} 4$ acetylation and the epigenetic reader Brd4 are critical regulators of pluripotency in embryonic stem cells. BMC Genomics 17, 95.

Grippo, P.J., Nowlin, P.S., Demeure, M.J., Longnecker, D.S., and Sandgren, E.P. (2003). Preinvasive pancreatic neoplasia of ductal phenotype induced by acinar cell targeting of mutant Kras in transgenic mice. Cancer Res. 63, 2016-2019.

Guerra, C., Schuhmacher, A.J., Cañamero, M., Grippo, P.J., Verdaguer, L., Pérez-Gallego, L., Dubus, P., Sandgren, E.P., and Barbacid, M. (2007). Chronic Pancreatitis Is Essential for Induction of Pancreatic Ductal Adenocarcinoma by K-Ras Oncogenes in Adult Mice. Cancer Cell 11, 291-302.

Hamacher, R., Schmid, R.M., Saur, D., and Schneider, G. (2008). Apoptotic pathways in pancreatic ductal adenocarcinoma. Mol. Cancer 7, 64.

Hamilton, G., Hochmair, M., Rath, B., Klameth, L., and Zeillinger, R. (2016). Small cell lung cancer: circulating tumor cells of extended stage patients express a mesenchymal-epithelial transition phenotype. Cell Adhes. Migr. 0. 
Hara, H., Takeda, T., Yamamoto, N., Furuya, K., Hirose, K., Kamiya, T., and Adachi, T. (2013). Zinc-induced modulation of SRSF6 activity alters Bim splicing to promote generation of the most potent apoptotic isoform BimS. FEBS J. 280, 3313-3327.

Harada, H., and Grant, S. (2012). Targeting the regulatory machinery of BIM for cancer therapy. Crit. Rev. Eukaryot. Gene Expr. 22, 117-129.

Harada, H., Quearry, B., Ruiz-Vela, A., and Korsmeyer, S.J. (2004). Survival factor-induced extracellular signal-regulated kinase phosphorylates BIM, inhibiting its association with BAX and proapoptotic activity. Proc. Natl. Acad. Sci. U. S. A. $101,15313-15317$.

Hartman, D.J., and Krasinskas, A.M. (2012a). Assessing treatment effect in pancreatic cancer. Arch. Pathol. Lab. Med. 136, 100-109.

Hartman, D.J., and Krasinskas, A.M. (2012b). Assessing treatment effect in pancreatic cancer. Arch. Pathol. Lab. Med. 136, 100-109.

Heinz, S., Romanoski, C.E., Benner, C., and Glass, C.K. (2015). The selection and function of cell type-specific enhancers. Nat. Rev. Mol. Cell Biol. 16, 144154.

Hermann, P.C., Huber, S.L., Herrler, T., Aicher, A., Ellwart, J.W., Guba, M., Bruns, C.J., and Heeschen, C. (2007). Distinct Populations of Cancer Stem Cells Determine Tumor Growth and Metastatic Activity in Human Pancreatic Cancer. Cell Stem Cell 1, 313-323.

Hingorani, S.R., Wang, L., Multani, A.S., Combs, C., Deramaudt, T.B., Hruban, R.H., Rustgi, A.K., Chang, S., and Tuveson, D.A. (2005). Trp53R172H and KrasG12D cooperate to promote chromosomal instability and widely metastatic pancreatic ductal adenocarcinoma in mice. Cancer Cell 7, 469-483.

Hnilicová, J., Hozeifi, S., Stejskalová, E., Dušková, E., Poser, I., Humpolíčková, J., Hof, M., and Staněk, D. (2013). The C-terminal domain of Brd2 is important for chromatin interaction and regulation of transcription and alternative splicing. Mol. Biol. Cell 24, 3557-3568.

Hnisz, D., Abraham, B.J., Lee, T.I., Lau, A., Saint-André, V., Sigova, A.A., Hoke, H.A., and Young, R.A. (2013). Super-Enhancers in the Control of Cell Identity and Disease. Cell 155, 934-947.

Houzelstein, D., Bullock, S.L., Lynch, D.E., Grigorieva, E.F., Wilson, V.A., and Beddington, R.S.P. (2002). Growth and early postimplantation defects in mice deficient for the bromodomain-containing protein Brd4. Mol. Cell. Biol. 22, 37943802. 
Huang, C., Ma, R., Xu, Y., Li, N., Li, Z., Yue, J., Li, H., Guo, Y., and Qi, D. (2015). Wnt2 promotes non-small cell lung cancer progression by activating WNT/ $\beta$ catenin pathway. Am. J. Cancer Res. 5, 1032-1046.

Hussain, S.P. (2016). Pancreatic Cancer: Current Progress and Future Challenges. Int. J. Biol. Sci. 12, 270-272.

Imaizumi, A., Onishi, H., Yamasaki, A., Kawamoto, M., Umebayashi, M., Morisaki, T., and Hasumi, K. (2016). Hypoxic Conditions Promote Gemcitabine Sensitivity in a Pancreatic Cancer Stem Cell Line. Anticancer Res. 36, 653-657.

Infante, J.R., Fecher, L.A., Falchook, G.S., Nallapareddy, S., Gordon, M.S., Becerra, C., DeMarini, D.J., Cox, D.S., Xu, Y., Morris, S.R., et al. (2012). Safety, pharmacokinetic, pharmacodynamic, and efficacy data for the oral MEK inhibitor trametinib: a phase 1 dose-escalation trial. Lancet Oncol. 13, 773-781.

Infante, J.R., Papadopoulos, K.P., Bendell, J.C., Patnaik, A., Burris, H.A., Rasco, D., Jones, S.F., Smith, L., Cox, D.S., Durante, M., et al. (2013). A phase $1 \mathrm{~b}$ study of trametinib, an oral Mitogen-activated protein kinase kinase (MEK) inhibitor, in combination with gemcitabine in advanced solid tumours. Eur. J. Cancer Oxf. Engl. 1990 49, 2077-2085.

Infante, J.R., Somer, B.G., Park, J.O., Li, C.-P., Scheulen, M.E., Kasubhai, S.M., Oh, D.-Y., Liu, Y., Redhu, S., Steplewski, K., et al. (2014). A randomised, doubleblind, placebo-controlled trial of trametinib, an oral MEK inhibitor, in combination with gemcitabine for patients with untreated metastatic adenocarcinoma of the pancreas. Eur. J. Cancer Oxf. Engl. 1990 50, 2072-2081.

Jang, M.K., Mochizuki, K., Zhou, M., Jeong, H.-S., Brady, J.N., and Ozato, K. (2005). The bromodomain protein Brd4 is a positive regulatory component of $\mathrm{P}$ TEFb and stimulates RNA polymerase II-dependent transcription. Mol. Cell 19, 523-534.

Jayson, G.C., Kerbel, R., Ellis, L.M., and Harris, A.L. (2016). Antiangiogenic therapy in oncology: current status and future directions. Lancet Lond. Engl.

Jemal, A., Bray, F., Center, M.M., Ferlay, J., Ward, E., and Forman, D. (2011). Global cancer statistics. CA. Cancer J. Clin. 61, 69-90.

Jenuwein, T., and Allis, C.D. (2001). Translating the histone code. Science 293, 1074-1080.

Jiang, Y.W., Veschambre, P., Erdjument-Bromage, H., Tempst, P., Conaway, J.W., Conaway, R.C., and Kornberg, R.D. (1998). Mammalian mediator of transcriptional regulation and its possible role as an end-point of signal transduction pathways. Proc. Natl. Acad. Sci. U. S. A. 95, 8538-8543. 
Juan, W.C., Roca, X., and Ong, S.T. (2014). Identification of cis-acting elements and splicing factors involved in the regulation of BIM Pre-mRNA splicing. PloS One 9, e95210.

Kalcheim, C. (2015). Epithelial-Mesenchymal Transitions during Neural Crest and Somite Development. J. Clin. Med. 5.

Kalluri, R., and Weinberg, R.A. (2009). The basics of epithelial-mesenchymal transition. J. Clin. Invest. 119, 1420-1428.

Kanda, M., Sadakari, Y., Borges, M., Topazian, M., Farrell, J., Syngal, S., Lee, J., Kamel, I., Lennon, A.M., Knight, S., et al. (2013). Mutant TP53 in duodenal samples of pancreatic juice from patients with pancreatic cancer or high-grade dysplasia. Clin. Gastroenterol. Hepatol. Off. Clin. Pract. J. Am. Gastroenterol. Assoc. 11, 719-730.e5.

Kang, Y., and Massagué, J. (2004). Epithelial-mesenchymal transitions: twist in development and metastasis. Cell 118, 277-279.

Kang, Y., Ling, J., Suzuki, R., Roife, D., Chopin-Laly, X., Truty, M.J., Chatterjee, D., Wang, H., Thomas, R.M., Katz, M.H., et al. (2014). SMAD4 Regulates Cell Motility through Transcription of N-Cadherin in Human Pancreatic Ductal Epithelium. PLOS ONE 9, e107948.

Kanno, T., Kanno, Y., LeRoy, G., Campos, E., Sun, H.-W., Brooks, S.R., Vahedi, G., Heightman, T.D., Garcia, B.A., Reinberg, D., et al. (2014). BRD4 assists elongation of both coding and enhancer RNAs by interacting with acetylated histones. Nat. Struct. Mol. Biol. 21, 1047-1057.

Karin, M., and Greten, F.R. (2005). NF-kappaB: linking inflammation and immunity to cancer development and progression. Nat. Rev. Immunol. 5, 749759.

Kashatus, J.A., Nascimento, A., Myers, L.J., Sher, A., Byrne, F.L., Hoehn, K.L., Counter, C.M., and Kashatus, D.F. (2015). Erk2 Phosphorylation of Drp1 Promotes Mitochondrial Fission and MAPK-Driven Tumor Growth. Mol. Cell 57, 537-551.

Kaufhold, S., and Bonavida, B. (2014). Central role of Snail1 in the regulation of EMT and resistance in cancer: a target for therapeutic intervention. J. Exp. Clin. Cancer Res. CR 33, 62.

Kelly, K.A., Hollingsworth, M.A., Brand, R.E., Liu, C.H., Singh, V.K., Srivastava, S., Wasan, A.D., Yadav, D., and Andersen, D.K. (2015). Advances in Biomedical Imaging, Bioengineering, and Related Technologies for the Development of Biomarkers of Pancreatic Disease: Summary of a National Institute of Diabetes and Digestive and Kidney Diseases and National Institute of Biomedical Imaging and Bioengineering Workshop. Pancreas 44, 1185-1194. 
Kempf, E., Rousseau, B., Besse, B., and Paz-Ares, L. (2016). KRAS oncogene in lung cancer: focus on molecularly driven clinical trials. Eur. Respir. Rev. Off. J. Eur. Respir. Soc. 25, 71-76.

Kim, I.-W., Han, N., Kim, M.G., Kim, T., and Oh, J.M. (2015). Copy number variability analysis of pharmacogenes in patients with lymphoma, leukemia, hepatocellular, and lung carcinoma using The Cancer Genome Atlas data. Pharmacogenet. Genomics 25, 1-7.

Kim, S.-H., Kim, J.M., Shin, M., Kim, C.W., Huang, S.-M., Kang, D.W., Suh, K.S., Yi, E.S., and Kim, K.-H. (2012). Correlation of epithelial-mesenchymal transition markers with clinicopathologic parameters in adenocarcinomas and squamous cell carcinoma of the lung. Histol. Histopathol. 27, 581-591.

Kim, S.-Y., Hong, S.-H., Basse, P.H., Wu, C., Bartlett, D.L., Kwon, Y.T., and Lee, Y.J. (2016). Cancer Stem Cells Protect Non-Stem Cells from Anoikis: Bystander Effects. J. Cell. Biochem.

Köenig, A., Linhart, T., Schlengemann, K., Reutlinger, K., Wegele, J., Adler, G., Singh, G., Hofmann, L., Kunsch, S., Büch, T., et al. (2010). NFAT-induced histone acetylation relay switch promotes c-Myc-dependent growth in pancreatic cancer cells. Gastroenterology 138, 1189-1199.e1-e2.

Kranenburg, O. (2005). The KRAS oncogene: past, present, and future. Biochim. Biophys. Acta 1756, 81-82.

Krantz, S.B., Shields, M.A., Dangi-Garimella, S., Bentrem, D.J., and Munshi, H.G. (2010). Contribution of Epithelial-Mesenchymal Transition to Pancreatic Cancer Progression. Cancers 2, 2084-2097.

Ku, C.S., Naidoo, N., Wu, M., and Soong, R. (2011). Studying the epigenome using next generation sequencing. J. Med. Genet. 48, 721-730.

Kumar, M., Allison, D.F., Baranova, N.N., Wamsley, J.J., Katz, A.J., Bekiranov, S., Jones, D.R., and Mayo, M.W. (2013). NF-kB regulates mesenchymal transition for the induction of non-small cell lung cancer initiating cells. PloS One 8, e68597.

Kurrey, N.K., Jalgaonkar, S.P., Joglekar, A.V., Ghanate, A.D., Chaskar, P.D., Doiphode, R.Y., and Bapat, S.A. (2009). Snail and slug mediate radioresistance and chemoresistance by antagonizing p53-mediated apoptosis and acquiring a stem-like phenotype in ovarian cancer cells. Stem Cells Dayt. Ohio 27, 20592068.

Lai, F., Jiang, C.C., Farrelly, M.L., Zhang, X.D., and Hersey, P. (2012). Evidence for upregulation of Bim and the splicing factor SRp55 in melanoma cells from patients treated with selective BRAF inhibitors. Melanoma Res. 22, 244-251. 
Lamonica, J.M., Deng, W., Kadauke, S., Campbell, A.E., Gamsjaeger, R., Wang, H., Cheng, Y., Billin, A.N., Hardison, R.C., Mackay, J.P., et al. (2011).

Bromodomain protein Brd3 associates with acetylated GATA1 to promote its chromatin occupancy at erythroid target genes. Proc. Natl. Acad. Sci. U. S. A. 108, E159-E168.

Lamouille, S., Xu, J., and Derynck, R. (2014). Molecular mechanisms of epithelial-mesenchymal transition. Nat. Rev. Mol. Cell Biol. 15, 178-196.

Lee, S., Rellinger, E.J., Kim, K.W., Craig, B.T., Romain, C.V., Qiao, J., and Chung, D.H. (2015). Bromodomain and extraterminal inhibition blocks tumor progression and promotes differentiation in neuroblastoma. Surgery 158, 819826.

LeRoy, G., Rickards, B., and Flint, S.J. (2008). The double bromodomain proteins Brd2 and Brd3 couple histone acetylation to transcription. Mol. Cell 30, $51-60$.

Li, C., Heidt, D.G., Dalerba, P., Burant, C.F., Zhang, L., Adsay, V., Wicha, M., Clarke, M.F., and Simeone, D.M. (2007). Identification of pancreatic cancer stem cells. Cancer Res. 67, 1030-1037.

Li, G.-Q., Guo, W.-Z., Zhang, Y., Seng, J.-J., Zhang, H.-P., Ma, X.-X., Zhang, G., Li, J., Yan, B., Tang, H.-W., et al. (2016). Suppression of BRD4 inhibits human hepatocellular carcinoma by repressing MYC and enhancing BIM expression. Oncotarget 7, 2462-2474.

Li, M., Zhang, B., Zhang, Z., Liu, X., Qi, X., Zhao, J., Jiang, Y., Zhai, H., Ji, Y., and Luo, D. (2014). Stem cell-like circulating tumor cells indicate poor prognosis in gastric cancer. BioMed Res. Int. 2014, 981261.

Li, Y., Ke, Q., Shao, Y., Zhu, G., Li, Y., Geng, N., Jin, F., and Li, F. (2015). GATA1 induces epithelial-mesenchymal transition in breast cancer cells through PAK5 oncogenic signaling. Oncotarget 6, 4345-4356.

Liang, C.-C., Park, A.Y., and Guan, J.-L. (2007). In vitro scratch assay: a convenient and inexpensive method for analysis of cell migration in vitro. Nat. Protoc. 2, 329-333.

Liao, A., Wang, W., Sun, D., Jiang, Y., Tian, S., Li, J., Yang, X., and Shi, R. (2015). Bone morphogenetic protein 2 mediates epithelial-mesenchymal transition via AKT and ERK signaling pathways in gastric cancer. Tumour Biol. J. Int. Soc. Oncodevelopmental Biol. Med. 36, 2773-2778.

Lindberg, J.M., Newhook, T.E., Adair, S.J., Walters, D.M., Kim, A.J., Stelow, E.B., Parsons, J.T., and Bauer, T.W. (2014). Co-Treatment with Panitumumab and Trastuzumab Augments Response to the MEK Inhibitor Trametinib in a Patient-Derived Xenograft Model of Pancreatic Cancer. Neoplasia 16, 562-571. 
Lockwood, W.W., Zejnullahu, K., Bradner, J.E., and Varmus, H. (2012).

Sensitivity of human lung adenocarcinoma cell lines to targeted inhibition of BET epigenetic signaling proteins. Proc. Natl. Acad. Sci. U. S. A. 109, 19408-19413.

Luo, W., Li, S., Peng, B., Ye, Y., Deng, X., and Yao, K. (2013). Embryonic stem cells markers SOX2, OCT4 and Nanog expression and their correlations with epithelial-mesenchymal transition in nasopharyngeal carcinoma. PloS One 8 , e56324.

Maione, F., Capano, S., Regano, D., Zentilin, L., Giacca, M., Casanovas, O., Bussolino, F., Serini, G., and Giraudo, E. (2012). Semaphorin 3A overcomes cancer hypoxia and metastatic dissemination induced by antiangiogenic treatment in mice. J. Clin. Invest. 122, 1832-1848.

Maione, P., Sacco, P.C., Casaluce, F., Sgambato, A., Santabarbara, G., Rossi, A., and Gridelli, C. (2016). Overcoming resistance to EGFR inhibitors in NSCLC. Rev. Recent Clin. Trials.

Mani, S.A., Guo, W., Liao, M.-J., Eaton, E.N., Ayyanan, A., Zhou, A.Y., Brooks, M., Reinhard, F., Zhang, C.C., Shipitsin, M., et al. (2008). The epithelialmesenchymal transition generates cells with properties of stem cells. Cell 133, 704-715.

Mao, Y., Xu, J., Li, Z., Zhang, N., Yin, H., and Liu, Z. (2013). The role of nuclear $\beta$-catenin accumulation in the Twist2-induced ovarian cancer EMT. PloS One 8 , e78200.

Marshall, G.M., Gherardi, S., Xu, N., Neiron, Z., Trahair, T., Scarlett, C.J., Chang, D.K., Liu, P.Y., Jankowski, K., Iraci, N., et al. (2010). Transcriptional upregulation of histone deacetylase 2 promotes Myc-induced oncogenic effects. Oncogene 29, 5957-5968.

Mattei, F., Liverani, S., Guida, F., Matrat, M., Cenée, S., Azizi, L., Menvielle, G., Sanchez, M., Pilorget, C., Lapôtre-Ledoux, B., et al. (2016). Multidimensional analysis of the effect of occupational exposure to organic solvents on lung cancer risk: the ICARE study. Occup. Environ. Med.

McCleary-Wheeler, A.L., Lomberk, G.A., Weiss, F.U., Schneider, G., Fabbri, M., Poshusta, T.L., Dusetti, N.J., Baumgart, S., lovanna, J.L., Ellenrieder, V., et al. (2013). Insights into the epigenetic mechanisms controlling pancreatic carcinogenesis. Cancer Lett. 328, 212-221.

McCubrey, J.A., Steelman, L.S., Chappell, W.H., Abrams, S.L., Wong, E.W.T., Chang, F., Lehmann, B., Terrian, D.M., Milella, M., Tafuri, A., et al. (2007). Roles of the Raf/MEK/ERK pathway in cell growth, malignant transformation and drug resistance. Biochim. Biophys. Acta 1773, 1263-1284. 
Meng, F.-D., Wei, J.-C., Qu, K., Wang, Z.-X., Wu, Q.-F., Tai, M.-H., Liu, H.-C., Zhang, R.-Y., and Liu, C. (2015). FoxM1 overexpression promotes epithelialmesenchymal transition and metastasis of hepatocellular carcinoma. World $\mathrm{J}$. Gastroenterol. 21, 196-213.

Mertz, J.A., Conery, A.R., Bryant, B.M., Sandy, P., Balasubramanian, S., Mele, D.A., Bergeron, L., and Sims, R.J. (2011). Targeting MYC dependence in cancer by inhibiting BET bromodomains. Proc. Natl. Acad. Sci. U. S. A. 108, 1666916674.

Di Micco, R., Fontanals-Cirera, B., Low, V., Ntziachristos, P., Yuen, S.K., Lovell, C.D., Dolgalev, I., Yonekubo, Y., Zhang, G., Rusinova, E., et al. (2014). Control of embryonic stem cell identity by BRD4-dependent transcriptional elongation of super-enhancer-associated pluripotency genes. Cell Rep. 9, 234-247.

Min, C., Eddy, S.F., Sherr, D.H., and Sonenshein, G.E. (2008). NF-kappaB and epithelial to mesenchymal transition of cancer. J. Cell. Biochem. 104, 733-744.

Mirsadraee, S., Oswal, D., Alizadeh, Y., Caulo, A., and van Beek, E.J. (2012). The 7th lung cancer TNM classification and staging system: Review of the changes and implications. World J. Radiol. 4, 128-134.

Modi, S., Kir, D., Banerjee, S., and Saluja, A. (2015). Control of Apoptosis in Treatment and Biology of Pancreatic Cancer. J. Cell. Biochem.

Morris, J.P., Cano, D.A., Sekine, S., Wang, S.C., and Hebrok, M. (2010). Betacatenin blocks Kras-dependent reprogramming of acini into pancreatic cancer precursor lesions in mice. J. Clin. Invest. 120, 508-520.

Mukhopadhyay, S., Panda, P.K., Sinha, N., Das, D.N., and Bhutia, S.K. (2014). Autophagy and apoptosis: where do they meet? Apoptosis Int. J. Program. Cell Death 19, 555-566.

Naidoo, J., and Drilon, A. (2016). KRAS-Mutant Lung Cancers in the Era of Targeted Therapy. Adv. Exp. Med. Biol. 893, 155-178.

National Lung Screening Trial Research Team, Aberle, D.R., Adams, A.M., Berg, C.D., Black, W.C., Clapp, J.D., Fagerstrom, R.M., Gareen, I.F., Gatsonis, C., Marcus, P.M., et al. (2011). Reduced lung-cancer mortality with low-dose computed tomographic screening. N. Engl. J. Med. 365, 395-409.

Neesse, A., Algül, H., Tuveson, D.A., and Gress, T.M. (2015). Stromal biology and therapy in pancreatic cancer: a changing paradigm. Gut 64, 1476-1484.

Neri, P., Ren, L., Azab, A.K., Brentnall, M., Gratton, K., Klimowicz, A.C., Lin, C., Duggan, P., Tassone, P., Mansoor, A., et al. (2011). Integrin $\beta 7$-mediated regulation of multiple myeloma cell adhesion, migration, and invasion. Blood 117, 6202-6213. 
Neureiter, D., Jäger, T., Ocker, M., and Kiesslich, T. (2014). Epigenetics and pancreatic cancer: pathophysiology and novel treatment aspects. World J. Gastroenterol. 20, 7830-7848.

Neuzillet, C., Tijeras-Raballand, A., de Mestier, L., Cros, J., Faivre, S., and Raymond, E. (2014). MEK in cancer and cancer therapy. Pharmacol. Ther. 141, 160-171.

Newhook, T.E., Lindberg, J.M., Adair, S.J., Kim, A.J., Stelow, E.B., Rahma, O.E., Parsons, J.T., and Bauer, T.W. (2016). Adjuvant Trametinib Delays the Outgrowth of Occult Pancreatic Cancer in a Mouse Model of Patient-Derived Liver Metastasis. Ann. Surg. Oncol.

Nicodeme, E., Jeffrey, K.L., Schaefer, U., Beinke, S., Dewell, S., Chung, C., Chandwani, R., Marazzi, I., Wilson, P., Coste, H., et al. (2010). Suppression of inflammation by a synthetic histone mimic. Nature 468, 1119-1123.

Nogueira, T.C., Paula, F.M., Villate, O., Colli, M.L., Moura, R.F., Cunha, D.A., Marselli, L., Marchetti, P., Cnop, M., Julier, C., et al. (2013). GLIS3, a susceptibility gene for type 1 and type 2 diabetes, modulates pancreatic beta cell apoptosis via regulation of a splice variant of the $\mathrm{BH}$-only protein Bim. PLoS Genet. 9, e1003532.

Noh, J., Sohn, J., Cho, J., Kang, D.R., Joo, S., Kim, C., and Shin, D.C. (2016). Residential radon and environmental burden of disease among Non-smokers. Ann. Occup. Environ. Med. 28, 12.

Normanno, N., De Luca, A., Maiello, M.R., Campiglio, M., Napolitano, M., Mancino, M., Carotenuto, A., Viglietto, G., and Menard, S. (2006). The MEK/MAPK pathway is involved in the resistance of breast cancer cells to the EGFR tyrosine kinase inhibitor gefitinib. J. Cell. Physiol. 207, 420-427.

Odero-Marah, V.A., Wang, R., Chu, G., Zayzafoon, M., Xu, J., Shi, C., Marshall, F.F., Zhau, H.E., and Chung, L.W.K. (2008). Receptor activator of NF-kappaB Ligand (RANKL) expression is associated with epithelial to mesenchymal transition in human prostate cancer cells. Cell Res. 18, 858-870.

Pasquier, J., Abu-Kaoud, N., Al Thani, H., and Rafii, A. (2015). Epithelial to Mesenchymal Transition in a Clinical Perspective. J. Oncol. 2015, 792182.

Patel, A.J., Liao, C.-P., Chen, Z., Liu, C., Wang, Y., and Le, L.Q. (2014). BET bromodomain inhibition triggers apoptosis of NF1-associated malignant peripheral nerve sheath tumors through Bim induction. Cell Rep. 6, 81-92.

Peng, J., Dong, W., Chen, L., Zou, T., Qi, Y., and Liu, Y. (2007). Brd2 is a TBPassociated protein and recruits TBP into E2F-1 transcriptional complex in response to serum stimulation. Mol. Cell. Biochem. 294, 45-54. 
Pott, S., and Lieb, J.D. (2015). What are super-enhancers? Nat. Genet. 47, 8-12.

Prieto, J., León, M., Ponsoda, X., Sendra, R., Bort, R., Ferrer-Lorente, R., Raya, A., López-García, C., and Torres, J. (2016). Early ERK1/2 activation promotes DRP1-dependent mitochondrial fission necessary for cell reprogramming. Nat. Commun. 7, 11124.

Puthalakath, H., O'Reilly, L.A., Gunn, P., Lee, L., Kelly, P.N., Huntington, N.D., Hughes, P.D., Michalak, E.M., McKimm-Breschkin, J., Motoyama, N., et al. (2007). ER stress triggers apoptosis by activating $\mathrm{BH} 3$-only protein Bim. Cell $129,1337-1349$.

Pylayeva-Gupta, Y., Grabocka, E., and Bar-Sagi, D. (2011). RAS oncogenes: weaving a tumorigenic web. Nat. Rev. Cancer 11, 761-774.

Quinn, B.A., Lee, N.A., Kegelman, T.P., Bhoopathi, P., Emdad, L., Das, S.K., Pellecchia, M., Sarkar, D., and Fisher, P.B. (2015). Chapter Eight - The Quest for an Effective Treatment for an Intractable Cancer: Established and Novel Therapies for Pancreatic Adenocarcinoma. In Advances in Cancer Research, P.B.F. and K.D. Tew, ed. (Academic Press), pp. 283-306.

Rahib, L., Smith, B.D., Aizenberg, R., Rosenzweig, A.B., Fleshman, J.M., and Matrisian, L.M. (2014). Projecting cancer incidence and deaths to 2030: the unexpected burden of thyroid, liver, and pancreas cancers in the United States. Cancer Res. 74, 2913-2921.

Raparia, K., Villa, C., DeCamp, M.M., Patel, J.D., and Mehta, M.P. (2013). Molecular profiling in non-small cell lung cancer: a step toward personalized medicine. Arch. Pathol. Lab. Med. 137, 481-491.

Renault, T.T., Floros, K.V., Elkholi, R., Corrigan, K.-A., Kushnareva, Y., Wieder, S.Y., Lindtner, C., Serasinghe, M.N., Asciolla, J.J., Buettner, C., et al. (2015). Mitochondrial Shape Governs BAX-Induced Membrane Permeabilization and Apoptosis. Mol. Cell 57, 69-82.

Richter-Larrea, J.A., Robles, E.F., Fresquet, V., Beltran, E., Rullan, A.J., Agirre, X., Calasanz, M.J., Panizo, C., Richter, J.A., Hernandez, J.M., et al. (2010). Reversion of epigenetically mediated BIM silencing overcomes chemoresistance in Burkitt lymphoma. Blood 116, 2531-2542.

Ryan, D.P., Hong, T.S., and Bardeesy, N. (2014). Pancreatic Adenocarcinoma. N. Engl. J. Med. 371, 1039-1049.

Rybinski, B., Franco-Barraza, J., and Cukierman, E. (2014). The wound healing, chronic fibrosis, and cancer progression triad. Physiol. Genomics 46, 223-244.

Ryerson, A.B., Eheman, C.R., Altekruse, S.F., Ward, J.W., Jemal, A., Sherman, R.L., Henley, S.J., Holtzman, D., Lake, A., Noone, A.-M., et al. (2016). Annual 
Report to the Nation on the Status of Cancer, 1975-2012, featuring the increasing incidence of liver cancer. Cancer n/a - n/a.

Ryu, H.S., Park, D.J., Kim, H.H., Kim, W.H., and Lee, H.S. (2012). Combination of epithelial-mesenchymal transition and cancer stem cell-like phenotypes has independent prognostic value in gastric cancer. Hum. Pathol. 43, 520-528.

Sahai, V., Kumar, K., Knab, L.M., Chow, C.R., Raza, S.S., Bentrem, D.J., Ebine, K., and Munshi, H.G. (2014). BET bromodomain inhibitors block growth of pancreatic cancer cells in three-dimensional collagen. Mol. Cancer Ther. 13, 1907-1917.

San José-Eneriz, E., Agirre, X., Jiménez-Velasco, A., Cordeu, L., Martín, V., Arqueros, V., Gárate, L., Fresquet, V., Cervantes, F., Martínez-Climent, J.A., et al. (2009). Epigenetic down-regulation of BIM expression is associated with reduced optimal responses to imatinib treatment in chronic myeloid leukaemia. Eur. J. Cancer Oxf. Engl. 1990 45, 1877-1889.

Sarchianaki, E., Derdas, S.P., Ntaoukakis, M., Vakonaki, E., Lagoudaki, E.D., Lasithiotaki, I., Sarchianaki, A., Koutsopoulos, A., Symvoulakis, E.K., Spandidos, D.A., et al. (2014). Detection and genotype analysis of human papillomavirus in non-small cell lung cancer patients. Tumour Biol. J. Int. Soc. Oncodevelopmental Biol. Med. 35, 3203-3209.

Saur, D., Seidler, B., Schneider, G., Algül, H., Beck, R., SenekowitschSchmidtke, R., Schwaiger, M., and Schmid, R.M. (2005). CXCR4 expression increases liver and lung metastasis in a mouse model of pancreatic cancer. Gastroenterology 129, 1237-1250.

Le Scodan, R., Mornex, F., Partensky, C., Mercier, C., Valette, P.-J., Ychou, M., Roy, P., and Scoazec, J.-Y. (2008). Histopathological Response to Preoperative Chemoradiation for Resectable Pancreatic Adenocarcinoma: The French Phase II FFCD 9704-SFRO Trial. Am. J. Clin. Oncol. 31, 545-552.

Sengupta, D., Kannan, A., Kern, M., Moreno, M.A., Vural, E., Stack, B., Suen, J.Y., Tackett, A.J., and Gao, L. (2015a). Disruption of BRD4 at H3K27Acenriched enhancer region correlates with decreased c-Myc expression in Merkel cell carcinoma. Epigenetics 10, 460-466.

Sengupta, S., Biarnes, M.C., Clarke, R., and Jordan, V.C. (2015b). Inhibition of BET proteins impairs estrogen-mediated growth and transcription in breast cancers by pausing RNA polymerase advancement. Breast Cancer Res. Treat. 150, 265-278.

Sequist, L. V, Waltman, B. a, Dias-Santagata, D., Digumarthy, S., Turke, A.B., Fidias, P., Bergethon, K., Shaw, A.T., Gettinger, S., Cosper, A.K., et al. (2011). Genotypic and histological evolution of lung cancers acquiring resistance to EGFR inhibitors. Sci. Transl. Med. 3, 75ra26. 
Shankar, J., and Nabi, I.R. (2015). Actin cytoskeleton regulation of epithelial mesenchymal transition in metastatic cancer cells. PloS One 10, e0119954.

Shi, J., Wang, Y., Zeng, L., Wu, Y., Deng, J., Zhang, Q., Lin, Y., Li, J., Kang, T., Tao, M., et al. (2014). Disrupting the interaction of BRD4 with diacetylated Twist suppresses tumorigenesis in basal-like breast cancer. Cancer Cell 25, 210-225.

Shimamura, T., Chen, Z., Soucheray, M., Carretero, J., Kikuchi, E., Tchaicha, J.H., Gao, Y., Cheng, K.A., Cohoon, T.J., Qi, J., et al. (2013). Efficacy of BET bromodomain inhibition in Kras-mutant non-small cell lung cancer. Clin. Cancer Res. Off. J. Am. Assoc. Cancer Res. 19, 6183-6192.

Shlyueva, D., Stampfel, G., and Stark, A. (2014). Transcriptional enhancers: from properties to genome-wide predictions. Nat. Rev. Genet. 15, 272-286.

Silverman, D.T., Schiffman, M., Everhart, J., Goldstein, A., Lillemoe, K.D., Swanson, G.M., Schwartz, A.G., Brown, L.M., Greenberg, R.S., Schoenberg, J.B., et al. (1999). Diabetes mellitus, other medical conditions and familial history of cancer as risk factors for pancreatic cancer. Br. J. Cancer 80, 1830-1837.

Sinn, M., Bahra, M., Denecke, T., Travis, S., Pelzer, U., and Riess, H. (2016). Perioperative treatment options in resectable pancreatic cancer - how to improve long-term survival. World J. Gastrointest. Oncol. 8, 248-257.

Sionov, R.V., Vlahopoulos, S.A., and Granot, Z. (2015). Regulation of Bim in Health and Disease. Oncotarget 6, 23058-23134.

Smith, B.N., and Bhowmick, N.A. (2016). Role of EMT in Metastasis and Therapy Resistance. J. Clin. Med. 5.

Song, J.S., Kim, Y.S., Kim, D.K., Park, S.I., and Jang, S.J. (2012). Global histone modification pattern associated with recurrence and disease-free survival in nonsmall cell lung cancer patients. Pathol. Int. 62, 182-190.

Spitz, F., and Furlong, E.E.M. (2012). Transcription factors: from enhancer binding to developmental control. Nat. Rev. Genet. 13, 613-626.

Sprenger, T., Conradi, L.-C., Beissbarth, T., Ermert, H., Homayounfar, K., Middel, P., Rüschoff, J., Wolff, H.A., Schüler, P., Ghadimi, B.M., et al. (2013).

Enrichment of CD133-expressing cells in rectal cancers treated with preoperative radiochemotherapy is an independent marker for metastasis and survival. Cancer 119, 26-35.

Strahl, B.D., and Allis, C.D. (2000). The language of covalent histone modifications. Nature 403, 41-45.

Stremitzer, S., Stift, J., Singh, J., Starlinger, P., Gruenberger, B., Tamandl, D., and Gruenberger, T. (2015). Histological response, pattern of tumor destruction 
and clinical outcome after neoadjuvant chemotherapy including bevacizumab or cetuximab in patients undergoing liver resection for colorectal liver metastases. Eur. J. Surg. Oncol. EJSO 41, 868-874.

Sugano, T., Seike, M., Noro, R., Soeno, C., Chiba, M., Zou, F., Nakamichi, S., Nishijima, N., Matsumoto, M., Miyanaga, A., et al. (2015). Inhibition of ABCB1 Overcomes Cancer Stem Cell-like Properties and Acquired Resistance to MET Inhibitors in Non-Small Cell Lung Cancer. Mol. Cancer Ther. 14, 2433-2440.

Sun, C., Hobor, S., Bertotti, A., Zecchin, D., Huang, S., Galimi, F., Cottino, F., Prahallad, A., Grernrum, W., Tzani, A., et al. (2014). Intrinsic resistance to MEK inhibition in KRAS mutant lung and colon cancer through transcriptional induction of ERBB3. Cell Rep. 7, 86-93.

Sun, L., Zhang, Q., Li, Y., Tang, N., and Qiu, X. (2015). CCL21/CCR7 upregulate vascular endothelial growth factor-D expression via ERK pathway in human non-small cell lung cancer cells. Int. J. Clin. Exp. Pathol. 8, 15729-15738.

Sweis, R.F., Thomas, S., Bank, B., Fishkin, P., Mooney, C., and Salgia, R. (2016). Concurrent EGFR Mutation and ALK Translocation in Non-Small Cell Lung Cancer. Curēus 8, e513.

Tampe, D., and Zeisberg, M. (2014). Potential approaches to reverse or repair renal fibrosis. Nat Rev Nephrol 10, 226-237.

Tan, N., Wong, M., Nannini, M.A., Hong, R., Lee, L.B., Price, S., Williams, K., Savy, P.P., Yue, P., Sampath, D., et al. (2013). Bcl-2/Bcl-xL inhibition increases the efficacy of MEK inhibition alone and in combination with PI3 kinase inhibition in lung and pancreatic tumor models. Mol. Cancer Ther. 12, 853-864.

Tögel, L., Nightingale, R., Chueh, A.C., Jayachandran, A., Tran, H., Phesse, T., Wu, R., Sieber, O.M., Arango, D., Dhillon, A.S., et al. (2016). Dual targeting of bromodomain and extra-terminal domain proteins, and WNT or MAPK signaling, inhibits c-MYC expression and proliferation of colorectal cancer cells. Mol. Cancer Ther.

Tolhuis, B., Palstra, R.J., Splinter, E., Grosveld, F., and de Laat, W. (2002). Looping and interaction between hypersensitive sites in the active beta-globin locus. Mol. Cell 10, 1453-1465.

Trauzold, A., Schmiedel, S., Röder, C., Tams, C., Christgen, M., Oestern, S., Arlt, A., Westphal, S., Kapischke, M., Ungefroren, H., et al. (2003). Multiple and synergistic deregulations of apoptosis-controlling genes in pancreatic carcinoma cells. Br. J. Cancer 89, 1714-1721.

Travis, W.D., Brambilla, E., Noguchi, M., Nicholson, A.G., Geisinger, K.R., Yatabe, Y., Beer, D.G., Powell, C.A., Riely, G.J., Van Schil, P.E., et al. (2011). International association for the study of lung cancer/american thoracic 
society/european respiratory society international multidisciplinary classification of lung adenocarcinoma. J. Thorac. Oncol. Off. Publ. Int. Assoc. Study Lung Cancer 6, 244-285.

Ungefroren, H., Voss, M., Jansen, M., Roeder, C., Henne-Bruns, D., Kremer, B., and Kalthoff, H. (1998). Human pancreatic adenocarcinomas express Fas and Fas ligand yet are resistant to Fas-mediated apoptosis. Cancer Res. 58, 17411749.

Valastyan, S., and Weinberg, R.A. (2011). Tumor metastasis: molecular insights and evolving paradigms. Cell 147, 275-292.

Venkataraman, S., Alimova, I., Balakrishnan, I., Harris, P., Birks, D.K., Griesinger, A., Amani, V., Cristiano, B., Remke, M., Taylor, M.D., et al. (2014). Inhibition of BRD4 attenuates tumor cell self-renewal and suppresses stem cell signaling in MYC driven medulloblastoma. Oncotarget 5, 2355-2371.

Verbeke, C., Löhr, M., Karlsson, J.S., and Del Chiaro, M. (2015). Pathology reporting of pancreatic cancer following neoadjuvant therapy: challenges and uncertainties. Cancer Treat. Rev. 41, 17-26.

Verma, M. (2015). The Role of Epigenomics in the Study of Cancer Biomarkers and in the Development of Diagnostic Tools. Adv. Exp. Med. Biol. 867, 59-80.

Vermeulen, L., de Sousa e Melo, F., Richel, D.J., and Medema, J.P. (2012). The developing cancer stem-cell model: clinical challenges and opportunities. Lancet Oncol. 13, e83-e89.

Vincent, T., Neve, E.P.A., Johnson, J.R., Kukalev, A., Rojo, F., Albanell, J., Pietras, K., Virtanen, I., Philipson, L., Leopold, P.L., et al. (2009). A SNAIL1SMAD3/4 transcriptional repressor complex promotes TGF-beta mediated epithelial-mesenchymal transition. Nat. Cell Biol. 11, 943-950.

Visel, A., Blow, M.J., Li, Z., Zhang, T., Akiyama, J.A., Holt, A., Plajzer-Frick, I., Shoukry, M., Wright, C., Chen, F., et al. (2009). ChIP-seq accurately predicts tissue-specific activity of enhancers. Nature 457, 854-858.

Walters, D.M., Stokes, J.B., Adair, S.J., Stelow, E.B., Borgman, C.A., Lowrey, B.T., Xin, W., Blais, E.M., Lee, J.K., Papin, J.A., et al. (2013a). Clinical, Molecular and Genetic Validation of a Murine Orthotopic Xenograft Model of Pancreatic Adenocarcinoma Using Fresh Human Specimens. PLoS ONE 8, e77065.

Walters, D.M., Lindberg, J.M., Adair, S.J., Newhook, T.E., Cowan, C.R., Stokes, J.B., Borgman, C.A., Stelow, E.B., Lowrey, B.T., Chopivsky, M.E., et al. (2013b). Inhibition of the growth of patient-derived pancreatic cancer xenografts with the MEK inhibitor trametinib is augmented by combined treatment with the epidermal growth factor receptor/HER2 inhibitor lapatinib. Neoplasia N. Y. N 15, 143-155. 
Wamsley, J.J., Kumar, M., Allison, D.F., Clift, S.H., Holzknecht, C.M., Szymura, S.J., Hoang, S.A., Xu, X., Moskaluk, C.A., Jones, D.R., et al. (2015). Activin upregulation by NF-KB is required to maintain mesenchymal features of cancer stem-like cells in non-small cell lung cancer. Cancer Res. 75, 426-435.

Wang, J., Zhou, J.-Y., and Wu, G.S. (2011). Bim protein degradation contributes to cisplatin resistance. J. Biol. Chem. 286, 22384-22392.

Wang, S., Cao, W., Xing, H., Chen, Y.L., Li, Q., Shen, T., Jiang, C., and Zhu, D. (2016). Activation of ERK pathway is required for 15-HETE-induced angiogenesis in human umbilical vascular endothelial cells. J. Recept. Signal Transduct. Res. 36, 225-232.

Wang, Z., Li, Y., Kong, D., Banerjee, S., Ahmad, A., Azmi, A.S., Ali, S., Abbruzzese, J.L., Gallick, G.E., and Sarkar, F.H. (2009). Acquisition of epithelialmesenchymal transition phenotype of gemcitabine-resistant pancreatic cancer cells is linked with activation of the notch signaling pathway. Cancer Res. 69, 2400-2407.

Wei, Y., Xia, W., Zhang, Z., Liu, J., Wang, H., Adsay, N.V., Albarracin, C., Yu, D., Abbruzzese, J.L., Mills, G.B., et al. (2008). Loss of trimethylation at lysine 27 of histone $\mathrm{H} 3$ is a predictor of poor outcome in breast, ovarian, and pancreatic cancers. Mol. Carcinog. 47, 701-706.

Westphal, S., and Kalthoff, H. (2003). Apoptosis: targets in pancreatic cancer. Mol. Cancer 2, 6.

White, R.R., Xie, H.B., Gottfried, M.R., Czito, B.G., Hurwitz, H.I., Morse, M.A., Blobe, G.C., Paulson, E.K., Baillie, J., Branch, M.S., et al. (2005). Significance of Histological Response to Preoperative Chemoradiotherapy for Pancreatic Cancer. Ann. Surg. Oncol. 12, 214-221.

Whyte, W.A., Orlando, D.A., Hnisz, D., Abraham, B.J., Lin, C.Y., Kagey, M.H., Rahl, P.B., Lee, T.I., and Young, R.A. (2013). Master transcription factors and mediator establish super-enhancers at key cell identity genes. Cell 153, 307319.

Willimott, S., and Wagner, S.D. (2010). Post-transcriptional and post-translational regulation of Bcl2. Biochem. Soc. Trans. 38, 1571-1575.

Wright, C.J.M., and McCormack, P.L. (2013). Trametinib: first global approval. Drugs $73,1245-1254$.

Wu, S.-Y., and Chiang, C.-M. (2007). The double bromodomain-containing chromatin adaptor Brd4 and transcriptional regulation. J. Biol. Chem. 282, 13141-13145. 
Wu, X., Liu, D., Tao, D., Xiang, W., Xiao, X., Wang, M., Wang, L., Luo, G., Li, Y., Zeng, F., et al. (2016). BRD4 regulates EZH2 transcription through up-regulation of C-MYC and represents a novel therapeutic target in bladder cancer. Mol. Cancer Ther.

Wu, Y., Zhang, X., Salmon, M., Lin, X., and Zehner, Z.E. (2007). TGFß1 regulation of vimentin gene expression during differentiation of the $\mathrm{C} 2 \mathrm{C} 12$ skeletal myogenic cell line requires Smads, AP-1 and Sp1 family members. Biochim. Biophys. Acta BBA - Mol. Cell Res. 1773, 427-439.

Xu, R., Sato, N., Yanai, K., Akiyoshi, T., Nagai, S., Wada, J., Koga, K., Mibu, R., Nakamura, M., and Katano, M. (2009). Enhancement of Paclitaxel-induced Apoptosis by Inhibition of Mitogen-activated Protein Kinase Pathway in Colon Cancer Cells. Anticancer Res. 29, 261-270.

Xu, W., Wang, Z., Zhang, W., Qian, K., Li, H., Kong, D., Li, Y., and Tang, Y. (2015). Mutated K-ras activates CDK8 to stimulate the epithelial-to-mesenchymal transition in pancreatic cancer in part via the $\mathrm{Wnt} / \beta$-catenin signaling pathway. Cancer Lett. 356, 613-627.

Yang, C., Chen, H., Tan, G., Gao, W., Cheng, L., Jiang, X., Yu, L., and Tan, Y. (2013). FOXM1 promotes the epithelial to mesenchymal transition by stimulating the transcription of Slug in human breast cancer. Cancer Lett. 340, 104-112.

Yang, Z., Yik, J.H.N., Chen, R., He, N., Jang, M.K., Ozato, K., and Zhou, Q. (2005). Recruitment of P-TEFb for stimulation of transcriptional elongation by the bromodomain protein Brd4. Mol. Cell 19, 535-545.

Yano, T., Ito, K., Fukamachi, H., Chi, X.-Z., Wee, H.-J., Inoue, K., Ida, H., Bouillet, P., Strasser, A., Bae, S.-C., et al. (2006). The RUNX3 tumor suppressor upregulates Bim in gastric epithelial cells undergoing transforming growth factor beta-induced apoptosis. Mol. Cell. Biol. 26, 4474-4488.

Yoon, C., Park, D.J., Schmidt, B., Thomas, N.J., Lee, H.-J., Kim, T.S., Janjigian, Y.Y., Cohen, D.J., and Yoon, S.S. (2014). CD44 expression denotes a subpopulation of gastric cancer cells in which Hedgehog signaling promotes chemotherapy resistance. Clin. Cancer Res. Off. J. Am. Assoc. Cancer Res. 20, 3974-3988.

Zavadil, J., and Böttinger, E.P. (2005). TGF-beta and epithelial-to-mesenchymal transitions. Oncogene 24, 5764-5774.

Zhang, Z., Ma, P., Jing, Y., Yan, Y., Cai, M.-C., Zhang, M., Zhang, S., Peng, H., Ji, Z.-L., Di, W., et al. (2016). BET Bromodomain Inhibition as a Therapeutic Strategy in Ovarian Cancer by Downregulating FoxM1. Theranostics 6, 219-230.

Zhao, Y., Tan, J., Zhuang, L., Jiang, X., Liu, E.T., and Yu, Q. (2005). Inhibitors of histone deacetylases target the Rb-E2F1 pathway for apoptosis induction 
through activation of proapoptotic protein Bim. Proc. Natl. Acad. Sci. U. S. A. 102, 16090-16095.

Zheng, X., Carstens, J.L., Kim, J., Scheible, M., Kaye, J., Sugimoto, H., Wu, C.C., LeBleu, V.S., and Kalluri, R. (2015). Epithelial-to-mesenchymal transition is dispensable for metastasis but induces chemoresistance in pancreatic cancer. Nature 527, 525-530.

Zou, Z., Huang, B., Wu, X., Zhang, H., Qi, J., Bradner, J., Nair, S., and Chen, L.F. (2014). Brd4 maintains constitutively active NF-KB in cancer cells by binding to acetylated RelA. Oncogene 33, 2395-2404.

(2016). Cancer Facts \& Figures 2016. 